\title{
Epidemiology of Non-communicable Diseases and Risk factors in South African Adolescents and Youth Living with HIV: Implications for Integrated Prevention
}

Monika Kamkuemah ( $\nabla$ monika.kamkuemah@gmail.com )

University of Cape Town School of Public Health and Family Medicine https://orcid.org/0000-00027603-2792

\section{Blessings Gausi}

University of Cambridge

Tolu Oni

University of Cambridge

\section{Research}

Keywords: Adolescents, HIV, Non-communicable diseases, Mental health, Risk factors, Prevention, Epidemiology, Multimorbidity

Posted Date: September 22nd, 2020

DOI: https://doi.org/10.21203/rs.3.rs-75447/v1

License: (1) This work is licensed under a Creative Commons Attribution 4.0 International License.

Read Full License 


\section{Abstract \\ Background}

Adolescents and youth living with HIV (AYLHIV) face an elevated NCD risk resulting from HIV, psychosocial challenges and the complications of antiretroviral therapy (ART).

\section{Methods}

We conducted a cross-sectional study in six primary care facilities to investigate the prevalence of common NCDs and risk factors among AYLHIV in Cape Town, South Africa between March 2019 and January 2020. We collected information on pre-existent and previously unidentified NCDs and risk factors and collected sociodemographic information of adolescents and youth enrolled for primary HIV care. Characteristics between sexes and age groups were compared using parametric or non-parametric statistical tests.

\section{Results}

Three out of four participants were female, and the median age was 20.5 years (IQR 18.9-22.9). More than a quarter were not in education, employment or training (NEET) and $44 \%$ were multidimensionally poor. Our results show an existent burden of hypertension (5\%) and central obesity (37\%) as well as high levels of depression (43\%) and psychological distress symptoms (44\%). AYLHIV further self-reported high levels of household food insecurity (70\%), low fruit and vegetable consumption, high fast-food and sugarsweetened beverage intake, low nutritional knowledge and insufficient physical activity. Beyond the NCD risk attributable to HIV and ART, these multiple risk factors coupled with early initiation of high-risk behaviours like smoking, alcohol and drug use, further increase risk.

\section{Conclusions}

Our findings highlight the importance of integrated prevention with NCD risk screening as part of HIV care for AYLHIV and the need for early intervention on social, environmental and economic determinants of NCDs targeting adolescents and youth.

\section{Background}

\section{Global burden of NCDs and NCD risk factors}

Globally, non-communicable diseases (NCDs) are the leading cause of disability and premature mortality accounting for $71 \%$ of deaths worldwide and $80.6 \%$ of years lived with disability (YLD) in 2016 [1, 2]. The largest number of NCD deaths are caused by cardiovascular diseases, followed by cancers, chronic 
respiratory diseases and diabetes [1]. There has been a global epidemiological transition from communicable, maternal, neonatal, and nutritional deficiency (CMNN) diseases, to patterns of disease and mortality dominated by NCDs and injuries [3]. This transition is driven by multiple and interrelated factors including population growth, ageing, nutritional transition and increased urbanisation.

These factors are driving an increasing prevalence of NCD risk factors, with smoking, alcohol, insufficient physical activity and unhealthy diets contributing to the majority of the burden of NCDs [4]. For example, the global prevalence of daily smoking was $25.0 \%$ in men and $5.4 \%$ in women and $11.5 \%$ of global deaths were attributable to cigarette smoking in $2015[5,6]$; while alcohol was the leading cause of premature death and disability in people aged 15-49 years in 2016 [7, 8]. Globally one in four adults are not sufficiently physically active and physical inactivity accounted for 1 in 10 deaths worldwide [9] and 34.6 million disability adjusted life years (DALYs) in 2016 [10], making physical inactivity the fourth leading risk factor for mortality globally [6]. Unhealthy diet contributes to a range of NCDs specifically diabetes, cancers, and cardiovascular diseases. Dietary risk factors - low intake of fruits, vegetables and wholegrains; high intake of sodium and added sugar from processed foods and sugar-sweetened beverages - are another significant contributor to mortality and DALYs globally [11]. In 2017, one in five global deaths were associated with unhealthy diets [11].

Depression and anxiety disorders are also important leading causes of disability worldwide [12] and together with substance use disorders, are the largest contributors to disability in young adults [2]. Mental health disorders commonly co-occur with NCDs and NCD risk factors like tobacco use, unhealthy diet and physical inactivity, while harmful use of alcohol has been shown to cluster in people with mental disorders [13].

NCDs affect low- and middle-income countries (LMICs) disproportionately, with more than three-quarters of NCD deaths occurring in LMICs [14]. In 2017, the burden of NCDs in sub-Saharan Africa (SSA) was higher than the global average, and was almost equivalent to the total burden associated with CMNN diseases [15]. South Africa, an upper-middle income country, faces a quadruple disease burden with high levels of HIV/AIDS and tuberculosis (TB); CMNN diseases and deficiencies; NCDs as well as injuries and trauma [16]. In 2016, 51\% of deaths in South Africa were due to NCDs and the risk of premature mortality from NCDs was $26 \%$ [4].

\section{Rising levels of NCD risk factors with increased urbanisation in sub-Saharan Africa}

NCD risk factors are increasing in SSA where NCDs are emerging in the context of rapid urbanization, contributing to unhealthy diets and sedentary behaviour, especially amongst the poor residing in urban settings [17]. Most adults in SSA are exposed to at least one risk factor for NCDs, including tobacco, harmful alcohol use, unhealthy diet, physical inactivity, obesity, or high blood pressure [18].

Epidemiological analyses from 1980-2014 show that age-standardized average body mass index (BMI) and diabetes prevalence have increased steadily across Africa, at least as steeply as the global average [19]. In South Africa, the prevalence of NCD risk factors has increased over the last two decades. For 
example, the prevalence of overweight and obesity among adult South African women increased from 56-68\% between 1998 and 2016 [20]. One in three South African adults has hypertension, $16.2 \%$ smoke daily [21], and $18.3 \%$ engage in heavy episodic drinking [22]. South African adults aged 15 years and older who consume alcohol account for the fifth highest consumption rate of absolute alcohol in the world [22].

Young people are not immune to the scourge of NCDs. Of the global NCD deaths recorded in 2016, an estimated 1.7 million ( $4 \%$ of NCD deaths) occurred in people younger than 30 years [23]. Adolescents in SSA are growing up in a time of epidemiological and nutrition transition. Furthermore, many NCD-related risk factors are initiated or reinforced during adolescence, which makes it a critical period for intervention to prevent future disease.

\section{Life-course approach to NCD prevention}

A life-course perspective views human development as lifelong and recognizes that no life stage can be understood in isolation from others [24]. Many potentially harmful health behaviours begin in adolescence and many adult illnesses have been identified to have roots in childhood and adolescence [25]. About 70\% of premature adult deaths are caused by behaviours started in adolescence [25]. Smoking, which frequently begins during adolescence, will lead to an estimated 150 million tobaccorelated deaths during adulthood [26]. Childhood and adolescent obesity, which tend to persist into adulthood [27, 28], are associated with increased cardiovascular risk factors including hypertension, dyslipidaemia and hyperinsulinemia [29], which may culminate in adult cardiovascular morbidity and premature mortality [30,31]. Half of all lifetime cases of mental health disorders emerge by the age of 14 years and three-quarters are established by the age of 24 [32]. Adolescence is thus a critical period of both opportunity and risk accompanied by rapid physical and psychological development that sets the foundation for adult health, and the health of the next generation of children [33, 34].

\section{NCD risk factors in adolescents and youth in South Africa}

In 2018 there were 9.5 million young people aged 15-24 years in South Africa, making up 16\% of the population [35]. South Africa has the highest prevalence of childhood and adolescent overweight and obesity in sub-Saharan Africa (19\% of boys and $26 \%$ of girls under 20 years) rivalling that of many highincome countries [36]. Overweight and obesity rates have increased steadily especially amongst adolescents residing in urban settings [37]. Paradoxically, South Africa faces a double burden of malnutrition; with undernutrition and overweight or obesity coexisting in the same populations and even within the same households [37].

Moreover, over $40 \%$ of young people have insufficient levels of physical activity, with significantly more inactivity in females compared to males [38]. A third of adolescents and youth (11-20 years) reported drinking alcohol in the Youth Risk and Behaviour Survey (YRBS), with a quarter engaging in heavy episodic/ binge drinking, $18 \%$ were current smokers and $13 \%$ reported smoking cannabis in their lifetime [38]. Significantly more males than females reported alcohol use, binge drinking, smoking, and illicit drug use [38]. 
Despite these data which highlight the existing burden of NCD risk factors among adolescents and youth in South Africa, evidence on effective strategies to intervene for NCD prevention are less clear, particularly as adolescents and youth are less likely to routinely present in the health care system [39]. This raises the potential for NCD prevention intervention among adolescents who do regularly have contact with the healthcare system by virtue of having a chronic disease such as HIV.

\section{HIV and NCD multimorbidity and health system implications}

Of the 1.6 million adolescents (10-19 years) reported to be living with HIV worldwide in $2018,19 \%$ were South African [40]. Adolescents and youth living with HIV (AYLHIV) in South Africa bear a disproportionate burden of the HIV epidemic due partly to paediatric cohorts of mother-to-child transmission reaching adolescence [41] and increased sexual transmission amongst young adolescent girls associated with gender inequality and poverty [42].

AYLHIV face elevated health risks resulting from both infection with HIV and the complications of longterm antiretroviral treatment $[43,44]$. Inflammation as a result of HIV replication causes vascular abnormalities which can result in heart disease, stroke, altered glucose metabolism, malignancy, and neurologic disease $[45,46]$. Evidence on the increased risk of comorbidities, including osteoporosis, metabolic syndrome, cardiovascular and renal diseases, was initially documented in adult populations living with HIV, but similar patterns have since been identified in paediatric and adolescent cohorts [4749]. An excess burden of NCD risk factors has also been documented in people living with HIV (PLHIV) compared to the general population [50]; $49 \%$ are insufficiently physically active [51], 40-70\% are current smokers $[52,53]$ and $37-66 \%$ are current drinkers $[54,55]$. Almost half $(46 \%)$ of adult patients attending HIV clinics in Cape Town engaged in hazardous or harmful alcohol use, and $15 \%$ had problematic drug use [56].

In addition to physical health risks, AYLHIV face several psychosocial challenges associated with living with a highly stigmatized communicable disease [57]. HIV/AIDS in Africa occurs in the context of orphanhood, poverty and inconsistent guardianship, resulting in elevated risk of mental health problems [58-60]. AYLHIV are known to experience emotional and behavioural problems at higher rates in comparison to the general population, including post-traumatic stress disorder (PTSD), depression, severe anxiety and neurocognitive deficits which affect their school performance, career prospects, relationships and autonomy [57] and increase their susceptibility to risk-taking behaviours [61-63].

To date, NCD prevention efforts have focused on adults, despite evidence of an increasing burden of NCDs and NCD risk among PLHIV occurring at younger ages $[50,64,65]$. A previous study conducted in Cape Town demonstrated that, despite existing NCD comorbidity and risk factors in AYLHIV, there was limited integration of NCD screening and health promotion in adolescent and youth HIV healthcare services [66]. While a few studies have explored alcohol and tobacco use [67-69] in AYLHIV in SSA, there is overall data paucity on the burden of NCDs and other NCD risk factors in this population group in Africa. With the first cohorts of AYLHIV now entering adulthood and the move towards more 
comprehensive health services aimed at improving the quality of life of PLHIV, questions remain on the prevalence of NCD comorbidity and NCD risk factors in AYLHIV and the best way to ensure holistic adolescent health and well-being. The main aim of this study was to investigate the prevalence of common NCDs and associated NCD risk factors as well as knowledge on health risks among AYLHIV accessing primary health care.

\section{Methods}

\section{Study Setting and Population}

This was a cross-sectional study of AYLHIV aged 15-24 years attending primary care health facilities in Cape Town. The study was conducted in Cape Town, the second biggest metropolitan city in South Africa with an estimated population of 4.2 million people [70]. In 2016, adolescents and youth aged 1524 years comprised $16.3 \%$ of the population in the Western Cape province, within which Cape Town is located [71]. Cape Town is a city undergoing rapid nutritional and epidemiological transition with increasing prevalence of NCDs and high rates of associated risk factors like hypertension, obesity and smoking. The 2012 South African National Health and Nutrition Examination Survey (SANHANES) revealed that over $50 \%$ of Western Cape respondents were overweight or obese ( $43 \%$ males and $62.4 \%$ females), a third were smokers, and two-thirds were physically unfit [21]. The Western Cape also has the highest rates of tobacco smoking and alcohol use nationally for both men and women [21].

There has been an increasing trend in the prevalence of NCDs in the province with a hypertension prevalence of $30 \%$ and diabetes prevalence of $11 \%$ [21]. Provincial HIV prevalence in women and men aged 15-49 years was estimated at $18 \%$ in 2016 [20]. However, HIV prevalence amongst antenatal care (ANC) public sector clients within Cape Town Metro was slightly higher at 21.6\% in 2015 [72], and as high as $34.3 \%$ in Khayelitsha sub-district in 2012 [73]. NCDs and HIV are ranked amongst the top causes of premature deaths in the City of Cape Town [74], with the top five causes of death in 2016 ranked as diabetes mellitus, HIV, ischaemic heart diseases, cerebrovascular diseases and TB [75]. NCDs alone accounted for approximately two-thirds of all deaths in the Western Cape in 2015 and 50\% of the premature mortality burden [76].

The City of Cape Town delivers primary health care through four legislated substructures: Khayelitsha / Eastern, Mitchells Plain / Klipfontein, Western / Southern and Northern / Tygerberg [74]. Recruitment and data collection took place at six public sector HIV clinics, with 1-3 clinics selected within each of the four sub-structures (Fig. 1). These facilities serve patients living in peri-urban, high-density, low-income townships, collectively known as the Cape Flats, in the south-eastern part of Cape Town consisting of approximately 583380 predominantly black African and 'Coloured' mixed-ancestry populations [77]. The area consists of a mix of formal and informal dwellings with $56 \%$ of the population residing in formal dwellings, high unemployment levels (29\%) [77], and approximately $63 \%$ of households falling within the low-income bracket (earning < 280 USD per month) [78].

\section{Study Design and Sampling}


Sample size was determined using prevalence estimates from SANHANES-2012 for the 15-24 years age group [21]. The confidence level was set at $95 \%$, with a $5 \%$ degree of precision, and an obesity prevalence of $5.6 \%$. The minimum required sample size was 82 and the final sample size determined to accommodate a non-response rate of $5 \%$ was 86 participants.

Prior to data collection, we conducted stakeholder engagement, liaising with facility managers and adolescent healthcare providers. Each facility has different schedules and models of HIV care for AYLHIV necessitating customised recruitment plans. We previously described the method used to estimate the number of AYLHIV accessing care at each facility (author). Facilities in the Eastern and Khayelitsha substructure had the biggest number of adolescents and youth receiving care. The AYLHIV population size was used as a guide for the proportion required for recruitment at each facility. Given the challenges of recruiting adolescents, in order to reach the required sample size, a convenience sampling approach was used recruiting all AYLHIV who showed an interest in participating. Participant recruitment commenced in March 2019 after ethical clearance (HREC ref no: 520/2017) and approval from Provincial and Local Government Departments of Health. Recruitment and study procedures were then conducted after gaining written informed consent and assent (for participants under 18 years) at six facilities until January 2020 (Fig. 1). A total of 176 adolescents and youth were recruited and invited to participate, of which 92 attended the follow up appointment and were successfully interviewed, yielding a response rate of $52 \%$.

\section{Figure 1: Participant Recruitment and enrolment at respective facilities Study Procedures}

\section{Informed Consent Process}

Informed consent and assent forms were developed in English and translated into two local languages; isiXhosa and Afrikaans. At the first encounter, participants were given information sheets and a detailed overview of the study procedures and invited to participate during routine clinical visits. Prior to enrolment, those aged less than 18 years received the informed consent and assent forms in order to gain parental or caregiver approval and a return date was scheduled on which the interview would take place (usually the date of their next clinic appointment). Teach-back questions on the nature of the study were incorporated into the assent form to ascertain adequate understanding. Those of legal age (18-24 years) were immediately enrolled after signing the consent forms and a convenient, future time was scheduled for their interview if they were unable to participate on the same day.

On the scheduled appointment date, participants first underwent a 30-minute physical examination before completing a partially assisted self-administered questionnaire using electronic forms on a handheld Android tablet. The questionnaire, which was administered in English, took approximately 90 minutes to complete and included questions on socio-demographics, self-reported health status, family history, household characteristics, mental health, self-reported physical activity and dietary practices, use of alcohol, tobacco and illicit substances, and nutritional knowledge. 


\section{Physical Examination}

Physical examinations were carried out according to the study protocol Standard Operating Procedures. Height and weight were measured using a sliding balance weight-and-height measuring scale with participants barefoot and wearing light clothing. Height was measured to the nearest $0.5 \mathrm{~cm}$ and weight to the nearest $0.1 \mathrm{~kg}$. Waist and hip circumference were measured using stretch-resistant measuring tape according to the WHO STEPS Protocol [79]. Readings were taken to the nearest $0.1 \mathrm{~cm}$. Two measurements were taken and recorded on the electronic form of which an average was computed during analysis. For weight, height and waist- and hip circumference, if the two readings differed by more than $100 \mathrm{~g}, 2 \mathrm{~cm}$ and $0.1 \mathrm{~cm}$ respectively, a third measurement was taken, and the two closest measurements were recorded.

Sitting blood pressure (BP) and pulse were measured using a Rossmax automatic blood pressure monitor (Rossmax (Shanghai) Incorporation Ltd). Two readings were taken at least two minutes apart and the average was computed. The procedures were repeated in instances where there was a significant difference between readings (more than two units) and the closest two readings were recorded.

Participants were asked whether they had a family history of diabetes or experienced any of the following symptoms over the past three months: frequent urination, increased thirst, unexplained weight loss of more than $1.5 \mathrm{~kg}$ in the last month, unexplained fatigue and blurry vision. Random blood glucose was measured in those with a family history or reported symptoms using a point-of-care (POC) glucometer with reactive test-strips (Glucocheck Evolve ${ }^{\circledR}$ Homemed Pty).

Respiratory symptom screening was conducted determining any difficulty breathing or shortness of breath, prolonged cough for more than two weeks with sputum, chest tightness, noisy breathing (wheezing or whistling in the chest) or a history of asthma. Respiratory volume was measured in those reporting respiratory symptoms without known asthma or chronic respiratory conditions using a handheld peak flow meter with disposable mouth pieces. Several repeated attempts were made in accordance with standard guidelines [80]. If the variation between attempts was greater than 20 litres/minute [80], a further two attempts were made. The two largest values were recorded and the average computed.

\section{Data collection tools and definitions of composite measures}

\section{Structural and household risk factors}

Adolescence is often categorized into three primary developmental stages: early adolescence (1014 years), middle adolescence (15-17 years), and late adolescence / young adulthood (18-24 years) [81]. For the purposes of this study, we categorized participants into four age groups in line with these stages and further sub-divided the oldest age group as follows:15-17, 18-19, 20-21 and 22-24 years. 
Questions on socioeconomic status were derived from the 2011 South African Census Questionnaire's subset of variables used to measure multidimensional poverty [82]. The Youth Multidimensional Poverty Index (YMPI) is a multidimensional individual-level measure of poverty comprised of 11 weighted indicators in five dimensions: educational attainment, general health and functioning, living environment, household assets and employment, using the method by Alkire and Santos [83]. Each of the indicators is associated with a deprivation cut-off that defines whether a young person is deprived in that area [84]. The cut-offs for the educational attainment dimension are designed to coincide with key stages in the South African schooling system, allowing for a degree of delay in an individual's schooling career [84]. A deprivation score was calculated for each dimension and an overall composite score was derived from the weighted indicators. An individual is identified as being multidimensionally poor - MPI poor- if they are deprived in a third or more of the weighted indicators, with a composite score $\geq 33.3 \%$ [83]. The extent of poverty is measured by the percentage of deprivations experienced [85].

Food insecurity was measured using the Household Food Insecurity Access Scale (HFIAS) score. The HFIAS score is a continuous measure of the degree of food insecurity experienced in a household in the past month [86]. The participant is expected to answer for all members of the household and not just themselves. The higher the score, the more food insecurity the household experienced. Participants were categorized as living in food secure, mildly-, moderately-, or severely food insecure households depending on the severity and frequency of food insecurity experiences according to the HFIAS protocol [87].

\section{Behaviour and Knowledge}

Physical activity was assessed using the International Physical Activity Questionnaire (IPAQ) short form using the last seven days self-administered format [88] and graphic images of different kinds of vigorous and moderate-intensity forms of physical activity as an aid. The IPAQ has been validated in youth and adults in South Africa [89]. We used the Ainsworth et al. scoring algorithms to derive an average metabolic equivalent of task (MET) intensity level score for each type of physical activity: vigorous, moderate, walking and cycling [90]. Physical activity levels were further categorized according to the IPAQ scoring protocol into low, moderate and high [91]. Insufficient physical activity was defined as a score below 600 MET minutes/week according to the World Health Organization (WHO) recommendations [92]. The presence or absence of sedentary behaviour was dichotomized as spending three or more hours per day watching television, playing computer games, talking with friends or other sitting activities according to the Global School-based Student Health Survey criteria [93].

Dietary intake was assessed using a 23-item food frequency questionnaire (FFQ) adapted from the Health Behaviour in School-aged Children Survey [94]. Participants reported their 'usual' consumption frequency of 23 different food groups, with response categories ranging from 'never' to 'more than once a day'. The FFQ has been found to have moderate reliability and acceptable validity for assessing the consumption of most food groups among adolescents [95]. Dietary intake was summarized into weekly consumption frequencies: 1 , never; 2 , less than once a week; 3, once a week; 4, 2-4 days a week; 5, 5-6 days a week; 6 , once a day, every day; and 7 , every day, more than once. We estimated the proportion who 
ate fresh fruits and vegetables daily (once or more than once a day in the previous week) and frequently (on 4 or more days in the previous week) and the proportion of respondents who reported daily consumption of sugar-sweetened beverages (SSB), deep-fried foods, fast foods, salty snacks, and processed meats. Skipping breakfast was defined as eating breakfast on 0-2 days/week; semi-skipping, 3-4 days/week and not skipping, 5-7 days/week.

Tobacco, alcohol use and substance use were assessed using questions from the 2011 South African Youth Risk Behaviour Survey [38]. Heavy episodic or binge drinking was defined as drinking five or more drinks in succession on one or more days in the preceding month. Risky drinking was defined as binge drinking or underage drinking (any alcohol consumption below the legal age of 18 years).

Nutritional knowledge was assessed using a revised form of the General Nutrition Knowledge Questionnaire (GNKQ-R) [96]. The GNKQ-R has demonstrated internal consistency and is a valid and reliable measure of nutrition knowledge among young people [96]. A nutritional knowledge score was generated by totalling correct answers in four nutrition domains. The maximum possible score was 88 ; 18 for questions on "dietary recommendations", 36 for questions on food groups, 13 for "healthy food choices" and 21 for "associations between diet and disease". No norms exist to determine an adequate nutrition knowledge score [97]. Hence we computed the average nutrition knowledge score and compared the mean scores for each of the nutrition domains by gender and age groups comparable to a previous study of South African adults [98]. The percentage of respondents who answered the questions correctly in each domain was calculated.

\section{Comorbidities (pre-existing diagnoses or presence of symptoms)}

Respiratory disease was defined as self-reported pre-existing diagnosis of asthma, tuberculosis, bronchitis, or other lung disease. Experiencing any of the following symptoms in the preceding three months was characterized as presence of respiratory symptoms: prolonged cough with sputum for more than two weeks, chest tightness, shortness of breath, difficulty breathing; or having an abnormal peak flow reading. Similarly, diabetes was defined either as i) a pre-existing self-reported diagnosis; ii) a random blood glucose reading of $>7 \mathrm{mmol} / \mathrm{l}$ and having a family history of diabetes; or iii) a random blood glucose reading of $>7 \mathrm{mmol} / \mathrm{l}$ and experiencing any of the following diabetes-related symptoms over the past three months: frequent urination, increased thirst, unexplained weight loss of more than $1.5 \mathrm{~kg}$ in the last month, unexplained fatigue, blurry vision.

Depression and psychological distress were defined using symptom screening questions from the 10item Centre for Epidemiological Studies Short Depression Scale (CESD-10) $[99,100]$ and the Kessler Psychological Distress Scale (K10) [101]. Both tools have been validated in South African HIV-positive populations $[102,103]$ and in adolescents and young adults [104]. The CESD-10 scale assesses depressive symptoms in the past week, while the K10 scale assesses symptoms of distress during the previous 30 days. Depression was defined as a binary indicator using a cut-off score of 10 or more on the CESD-10 scale [99] and the likelihood of psychological distress was categorized according to the K10 
score: $\mathrm{K} 10<20$, mentally well; K10 20-24, likely to have mild psychological distress; K10 25-29, likely to have moderate psychological distress; K10 30-50, likely to have severe psychological distress [105].

\section{Measured clinical signs}

Overweight and obesity were categorized using standard BMI categories and cut-offs for central obesity. The criterion used to identify overweight and obesity in children and adolescents using growth charts corresponds to the criteria used for adults thus we opted for the latter for ease of comparability [106]. The abdominal obesity cut-off point for a high waist circumference (WC) was $\geq 102 \mathrm{~cm}$ in males and $\geq$ $88 \mathrm{~cm}$ in females and a waist-hip ratio (WHR) $>0.85$ for females and $>0.90$ for males [79]. Waist-to-hip ratio was calculated by dividing the waist circumference by the hip circumference in centimeters rounded to two decimal places. A threshold value of 0.5 for the waist-to-height ratio (WHtR) was used as a measure of central obesity, calculated by dividing the waist circumference by the height in $\mathrm{cm}$ [107]. Blood pressure (BP) was categorized according to the South African Hypertension practice guidelines [108]: Normal (systolic BP, SBP $<130 \mathrm{mmHg}$ and diastolic BP, DBP $<85 \mathrm{mmHg}$ ); elevated blood pressure (SBP $130-139 \mathrm{mmHg}$ or DBP $85-89 \mathrm{mmHg}$ ) and hypertension (SBP $\geq 140 \mathrm{mmHg}$ or DBP $\geq 90 \mathrm{mmHg}$ ) or a self-reported pre-existing diagnosis of hypertension.

\section{Data Management and Statistical Analysis}

Data from the physical examination and self-administered interviews were captured into an electronic form on an Android device using a unique anonymous study ID for each participant. At the end of each day, data was synced to a password-protected server only accessible to the study team. Data was then imported into Stata version 14.0 (StataCorp, College Station, Texas, USA) for cleaning and statistical analyses.

Graphical data exploration and Shapiro-Wilk's tests were used to test for normality of variables. All data were analysed and reported by sex and age group. Demographic and socioeconomic variables are described using summary statistics (frequencies, percentages, median and interquartile range (IQR)). Characteristics between sexes and age groups were compared using Pearson's $\chi 2$ and Fisher's exact tests for categorical measures. The Wilcoxon-Mann-Whitney test was used to compare medians between groups and the Kruskal-Wallis test was used for comparing continuous measures in more than two groups. Point prevalence estimates for comorbidities and symptoms were described and their exact binomial $95 \%$ confidence intervals were computed. All tests of significance were two-tailed and performed at the $5 \%$ significance level $(p<0.05)$.

\section{Ethical Considerations}

This study was approved by the University of Cape Town Faculty of Health Sciences Human Research Ethics Committee (HREC ref no: 520/2017). Approval was also granted by the Western Cape and City of Cape Town Departments of Health to access facilities for participant enrolment. Parents or legal guardians gave permission for their children to participate in the study, and participants provided 
informed assent (or consent if 18 years or older) for participation in the study. Participants received reimbursement for transport costs.

\section{Results}

\section{Socio-demographic characteristics, structural and household risk factors}

A total of 92 participants completed study procedures, $76 \%$ of whom were female. The median age was 20.5 years (IQR 18.9-22.9) and the majority of our respondents (60\%) were in the young adulthood stage between 20-24 years old (See Table 1). Over half (58\%) were enrolled in school/college/university or other tertiary education or training (median age 20.3 (IQR 18.8-22.7) years). The majority lived with a biological parent or a relative, and $2 \%$ reported living with non-family members in foster care or children's homes. More than a quarter of female respondents reported ever being pregnant (28\%) compared to $5 \%$ of male participants reporting ever impregnating someone. Of those ever pregnant, the majority $(70 \%)$ reported having one child. 
Table 1

Participant Socio-demographic, Structural and Household Characteristics by Gender

\begin{tabular}{|c|c|c|c|c|}
\hline Variable & $\begin{array}{l}\text { Description Median (IQR) or } n \\
(\%)(p<0.05)^{1}\end{array}$ & $\begin{array}{l}\text { Male: } n \\
=22 \\
(24 \%)\end{array}$ & $\begin{array}{l}\text { Female: } n \\
=70(76 \%)\end{array}$ & $\begin{array}{l}\text { Total } n \\
=92\end{array}$ \\
\hline Age (years) & & $\begin{array}{l}20.7 \\
(18.9- \\
21.6)\end{array}$ & $\begin{array}{l}20.4 \\
(19.0- \\
23.0)\end{array}$ & $\begin{array}{l}20.5 \\
(18.9- \\
22.9)\end{array}$ \\
\hline \multirow[t]{4}{*}{$\begin{array}{l}\text { Adolescent stage by age } \\
\text { group }\end{array}$} & $\begin{array}{l}\text { middle adolescence: } 15- \\
17 \text { years }\end{array}$ & $3(14 \%)$ & $10(15 \%)$ & $\begin{array}{l}13 \\
(14 \%)\end{array}$ \\
\hline & late adolescence: $18-19$ years & $5(23 \%)$ & $18(26 \%)$ & $\begin{array}{l}23 \\
(26 \%)\end{array}$ \\
\hline & young adulthood: $20-21$ years & $9(41 \%)$ & $16(24 \%)$ & $\begin{array}{l}25 \\
(28 \%)\end{array}$ \\
\hline & young adulthood: $22-24$ years & $5(23 \%)$ & $24(35 \%)$ & $\begin{array}{l}29 \\
(32 \%)\end{array}$ \\
\hline \multirow[t]{5}{*}{$\begin{array}{l}\text { Family structure- whom } \\
\text { they live with }\end{array}$} & Biological parents & $10(45 \%)$ & $35(50 \%)$ & $\begin{array}{l}45 \\
(49 \%)\end{array}$ \\
\hline & Grandparents & $4(18 \%)$ & $8(11 \%)$ & $\begin{array}{l}12 \\
(13 \%)\end{array}$ \\
\hline & Relative (aunt or uncle) & $6(27 \%)$ & $23(33 \%)$ & $\begin{array}{l}29 \\
(32 \%)\end{array}$ \\
\hline & Siblings & $15(68 \%)$ & $33(47 \%)$ & $\begin{array}{l}48 \\
(52 \%)\end{array}$ \\
\hline & $\begin{array}{l}\text { Non-family (foster care/ } \\
\text { children's home) }\end{array}$ & 0 & $2(3 \%)$ & $2(2 \%)$ \\
\hline \multicolumn{2}{|c|}{ Ever pregnant/ impregnated someone $(p=0.023)$} & $1(5 \%)$ & $19(28 \%)$ & $\begin{array}{l}20 \\
(22 \%)\end{array}$ \\
\hline \multirow[t]{3}{*}{ Parity $(n=20)$} & 0 children & 0 & $5(26 \%)$ & $5(25 \%)$ \\
\hline & 1 child & $1(100 \%)$ & $13(68 \%)$ & $\begin{array}{l}14 \\
(70 \%)\end{array}$ \\
\hline & 2 children & 0 & $1(5 \%)$ & $1(5 \%)$ \\
\hline \multirow[t]{2}{*}{$\begin{array}{l}\text { Current occupation/ } \\
\text { employment status }\end{array}$} & $\begin{array}{l}\text { In school/college/university/ } \\
\text { other tertiary education }\end{array}$ & $7(32 \%)$ & 37 (54\%) & $\begin{array}{l}44 \\
(48 \%)\end{array}$ \\
\hline & In training & $3(14 \%)$ & $6(9 \%)$ & $9(10 \%)$ \\
\hline
\end{tabular}

$P$-value shown if significant at $5 \%$ level: $p<0.05 ;^{2}$ Youth MPI poor: those with composite score > $33.3 \%{ }^{3}$ Individual living in a household that does not own more than two of: radio, TV, landline, mobile phone, bike, motorbike or refrigerator AND does not own a motor car or truck 


\begin{tabular}{|c|c|c|c|c|}
\hline \multirow[t]{3}{*}{ Variable } & $\begin{array}{l}\text { Description Median (IQR) or } n \\
(\%)(p<0.05)^{1}\end{array}$ & $\begin{array}{l}\text { Male: } n \\
=22 \\
(24 \%)\end{array}$ & $\begin{array}{l}\text { Female: } n \\
=70(76 \%)\end{array}$ & $\begin{array}{l}\text { Total } \mathrm{n} \\
=92\end{array}$ \\
\hline & Employed & $5(23 \%)$ & $8(11 \%)$ & $\begin{array}{l}13 \\
(14 \%)\end{array}$ \\
\hline & $\begin{array}{l}\text { Not in education, employment or } \\
\text { training (NEET) }\end{array}$ & $7(32 \%)$ & $18(26 \%)$ & $\begin{array}{l}25 \\
(27 \%)\end{array}$ \\
\hline \multicolumn{2}{|c|}{ Ever repeated a grade at school } & $14(64 \%)$ & $33(47 \%)$ & $\begin{array}{l}47 \\
(52 \%)\end{array}$ \\
\hline \multirow[t]{3}{*}{$\begin{array}{l}\text { Days absent from school } \\
\text { or work in past month }\end{array}$} & 0 days & $13(59 \%)$ & $34(49 \%)$ & $\begin{array}{l}47 \\
(52 \%)\end{array}$ \\
\hline & $1-2$ days & $7(32 \%)$ & $26(38 \%)$ & $\begin{array}{l}33 \\
(36 \%)\end{array}$ \\
\hline & 3 or more days & $2(9 \%)$ & $9(13 \%)$ & $\begin{array}{l}11 \\
(12 \%)\end{array}$ \\
\hline \multicolumn{2}{|c|}{ Youth multidimensionally poor ${ }^{2}(n=88)$} & $9(41 \%)$ & $30(45 \%)$ & $\begin{array}{l}39 \\
(44 \%)\end{array}$ \\
\hline \multicolumn{2}{|l|}{ Educational attainment } & $4(18 \%)$ & $16(24 \%)$ & $\begin{array}{l}20 \\
(22 \%)\end{array}$ \\
\hline & $\begin{array}{l}\text { Aged } 17-20 \& \text { completed less } \\
\text { than nine years of schooling }\end{array}$ & $1(5 \%)$ & $2(3 \%)$ & $3(3 \%)$ \\
\hline & $\begin{array}{l}\text { Aged } 21-24 \& \text { completed less } \\
\text { than matric or equivalent }\end{array}$ & $3(14 \%)$ & $14(21 \%)$ & $\begin{array}{l}17 \\
(19 \%)\end{array}$ \\
\hline \multicolumn{2}{|c|}{ General health and functioning } & $16(73 \%)$ & $46(67 \%)$ & $\begin{array}{l}62 \\
(68 \%)\end{array}$ \\
\hline & Difficulty hearing & $3(14 \%)$ & $13(19 \%)$ & $\begin{array}{l}16 \\
(18 \%)\end{array}$ \\
\hline & Difficulty seeing & $7(33 \%)$ & $26(39 \%)$ & $\begin{array}{l}33 \\
(38 \%)\end{array}$ \\
\hline & Difficulty moving around & $2(10 \%)$ & $15(22 \%)$ & $\begin{array}{l}17 \\
(20 \%)\end{array}$ \\
\hline & Difficulty concentrating & $12(57 \%)$ & $32(48 \%)$ & $\begin{array}{l}44 \\
(50 \%)\end{array}$ \\
\hline & Difficulty with self-care & 0 & $14(21 \%)$ & $\begin{array}{l}14 \\
(16 \%)\end{array}$ \\
\hline
\end{tabular}

P-value shown if significant at $5 \%$ level: $p<0.05 ;^{2}$ Youth MPI poor: those with composite score > $33.3 \% ;^{3}$ Individual living in a household that does not own more than two of: radio, TV, landline, mobile phone, bike, motorbike or refrigerator AND does not own a motor car or truck 


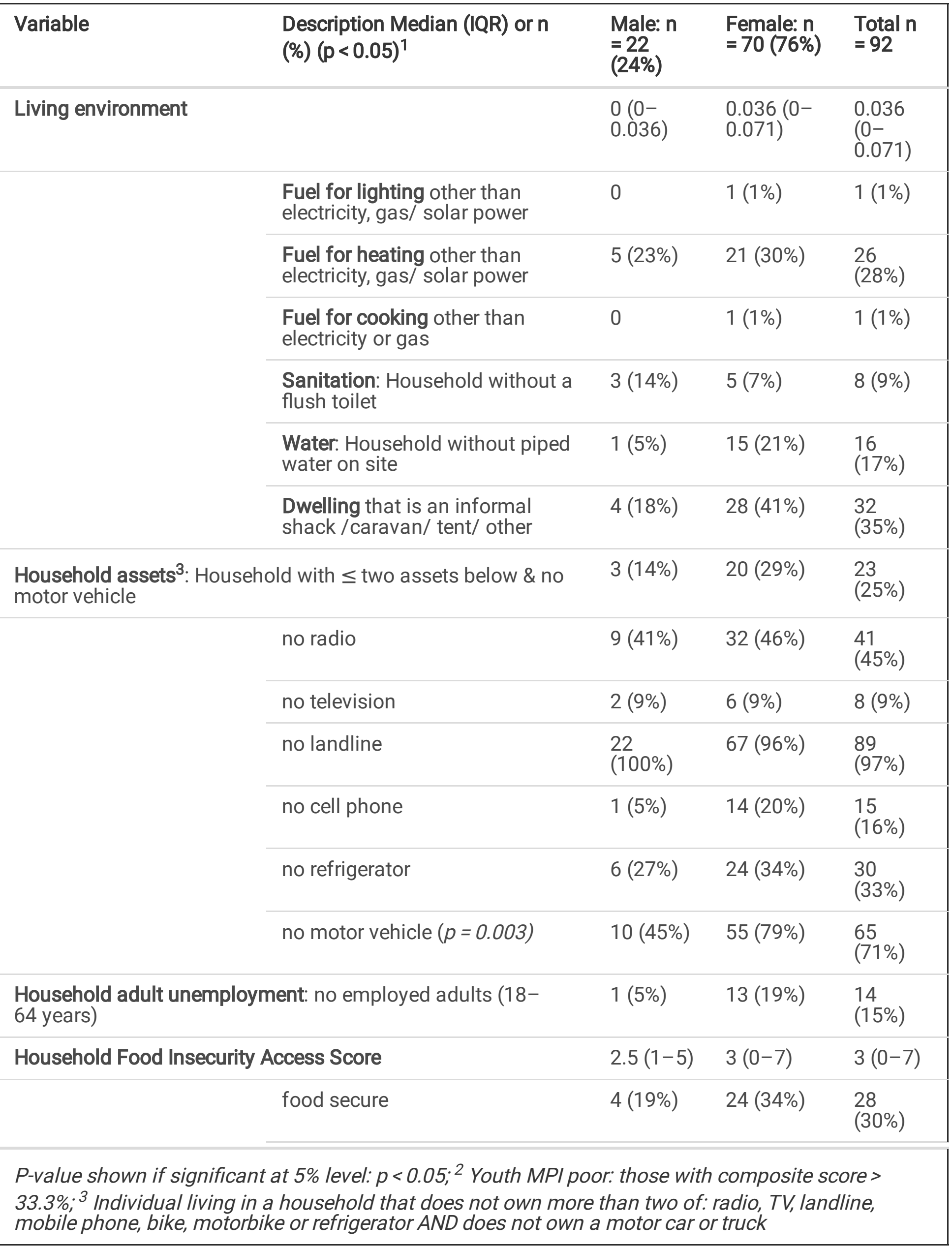




\begin{tabular}{|c|c|c|c|c|}
\hline Variable & $\begin{array}{l}\text { Description Median (IQR) or } n \\
(\%)(p<0.05)^{1}\end{array}$ & $\begin{array}{l}\text { Male: } n \\
=22 \\
(24 \%)\end{array}$ & $\begin{array}{l}\text { Female: } n \\
=70(76 \%)\end{array}$ & $\begin{array}{l}\text { Total } n \\
=92\end{array}$ \\
\hline & mildly food insecure & $7(32 \%)$ & $11(16 \%)$ & $\begin{array}{l}18 \\
(20 \%)\end{array}$ \\
\hline & moderately food insecure & $4(19 \%)$ & $7(10 \%)$ & $\begin{array}{l}11 \\
(12 \%)\end{array}$ \\
\hline & severely food insecure & $7(32 \%)$ & $28(40 \%)$ & $\begin{array}{l}35 \\
(38 \%)\end{array}$ \\
\hline \multicolumn{5}{|c|}{$\begin{array}{l}\text { P-value shown if significant at } 5 \% \text { level: } p<0.055^{2} \text { Youth MPI poor: those with composite score }> \\
33.3 \%{ }^{3} \text { Individual living in a household that does not own more than two of: radio, TV, landline, } \\
\text { mobile phone, bike, motorbike or refrigerator AND does not own a motor car or truck }\end{array}$} \\
\hline
\end{tabular}

\section{Multidimensional Poverty}

Overall, $44 \%$ of participants can be considered multi-dimensionally poor as they were deprived in a third or more of the five dimensions of MPI indicators as shown in Table 1. More than two-thirds (68\%) were deprived in the general health and functioning dimension on account of experiencing some difficulty with either hearing, sight, movement, concentration or with self-care. Fifty-nine percent were living-environment deprived with more than one-third living in informal housing, a quarter living in asset-deprived households, $28 \%$ living in households that do not use electricity, gas or solar power for heating and $17 \%$ living in households without piped water available on site. Almost a quarter $(22 \%)$ of our respondents were deprived in the educational attainment dimension, comprising 3\% of those aged 17-20 years who had completed less than nine years of schooling, and 19\% of those aged 21-24 years who did not have a high school degree (or the equivalent of 12 years of schooling). Overall, 39\% were economically deprived: 27\% were neither in education, employment or training (NEET) (median age 20.8 (IQR 19.8-23.3 years)), while $15 \%$ were living in households with no employed adults of working age and $3 \%$ were deprived in both economic indicators.

\section{Food insecurity}

Seventy percent of participants were living in food insecure households with $38 \%$ considered as severely food insecure: either having to cut back on meal size, number of meals or going a whole day and night without eating due to a lack of resources or receiving relief food in the last 30 days.

\section{Behaviour and Knowledge}

\section{Physical activity}

Overall, a third of respondents had insufficient levels of weekly physical activity, $41 \%$ had moderate levels and $27 \%$ had high levels of physical activity [91]. A greater proportion of males had high levels of physical activity compared to females (44\% versus $22 \%$ ). The total median MET-minutes of physical 
activity a week (total of all activities including active transport) was higher for males (2504.25 minutes) compared to females (1173 minutes), but this was not statistically significant (Table 2). The youngest age group reported the highest rates of vigorous-intensity physical activity (Table 3 ). Over two-thirds of all participants reported using active transport in the preceding week (mostly walking to school/work for at least ten minutes continuously). Almost half of respondents (49\%) spent more than three hours sedentary during a typical day, with no difference in sedentary behaviour by gender. 
Table 2

Physical Activity, Sedentary and Dietary Behaviour and Nutritional Knowledge of AYLHIV by Gender

\begin{tabular}{|c|c|c|c|c|}
\hline $\begin{array}{l}\text { Variable: Median (IQR) } \\
\text { or } n(\%)\end{array}$ & Description & $\begin{array}{l}\text { Male: } n= \\
19(22 \%)\end{array}$ & $\begin{array}{l}\text { Female: } n \\
=67(78 \%)\end{array}$ & $\begin{array}{l}\text { Total: } n= \\
86\end{array}$ \\
\hline \multirow[t]{3}{*}{$\begin{array}{l}\text { Vigorous-intensity } \\
\text { Physical activity }\end{array}$} & $\begin{array}{l}\text { Any vigorous-intensity PA for } \geq \\
10 \text { minutes }\end{array}$ & 11 (58\%) & $26(39 \%)$ & $37(43 \%)$ \\
\hline & $\begin{array}{l}\text { Time spent doing vigorous- } \\
\text { intensity PA per day in minutes }\end{array}$ & $\begin{array}{l}75(30- \\
240)\end{array}$ & $\begin{array}{l}60(30- \\
120)\end{array}$ & $\begin{array}{l}60(30- \\
120)\end{array}$ \\
\hline & $\begin{array}{l}\text { Vigorous intensity activity MET- } \\
\text { minutes/week }\end{array}$ & $\begin{array}{l}2400 \\
(1200- \\
4320)\end{array}$ & $\begin{array}{l}1680 \\
(480- \\
3840)\end{array}$ & $\begin{array}{l}1920 \\
(720- \\
4320)\end{array}$ \\
\hline \multirow[t]{3}{*}{$\begin{array}{l}\text { Moderate-intensity } \\
\text { Physical activity }\end{array}$} & $\begin{array}{l}\text { Any moderate-intensity PA for } \geq \\
10 \text { minutes }\end{array}$ & $15(79 \%)$ & $53(79 \%)$ & $68(79 \%)$ \\
\hline & $\begin{array}{l}\text { Time spent doing moderate- } \\
\text { intensity PA per day in mins }\end{array}$ & $\begin{array}{l}75(30- \\
120)\end{array}$ & $\begin{array}{l}60(30- \\
90)\end{array}$ & $\begin{array}{l}60(30- \\
90)\end{array}$ \\
\hline & $\begin{array}{l}\text { Moderate intensity activity MET- } \\
\text { minutes/week }\end{array}$ & $\begin{array}{l}1440 \\
(480- \\
1680)\end{array}$ & $\begin{array}{l}720(480- \\
1200)\end{array}$ & $\begin{array}{l}720 \\
(480- \\
1440)\end{array}$ \\
\hline \multirow[t]{3}{*}{ Active Travel } & $\begin{array}{l}\text { Walking or cycling for } \geq 10 \\
\text { minutes continuously for travel }\end{array}$ & $16(84 \%)$ & $45(67 \%)$ & $61(71 \%)$ \\
\hline & $\begin{array}{l}\text { Time spent walking or cycling on } \\
\text { a typical day in minutes }\end{array}$ & $\begin{array}{l}40(30- \\
60)\end{array}$ & $\begin{array}{l}30(30- \\
60)\end{array}$ & $\begin{array}{l}35(30- \\
60)\end{array}$ \\
\hline & Walking MET-minutes/week & $\begin{array}{l}610.5 \\
(396- \\
1386)\end{array}$ & $\begin{array}{l}495 \\
(247.5- \\
990)\end{array}$ & $\begin{array}{l}528 \\
(297- \\
990)\end{array}$ \\
\hline \multicolumn{2}{|c|}{ Total physical activity MET-minutes/week } & $\begin{array}{l}2504.25 \\
(690- \\
7146)\end{array}$ & $\begin{array}{l}1173 \\
(495- \\
2826)\end{array}$ & $\begin{array}{l}1215 \\
(495- \\
3348)\end{array}$ \\
\hline \multicolumn{2}{|c|}{$\begin{array}{l}\text { Insufficient physical activity (achieved < } 600 \text { MET-minutes } \\
\text { per week) }\end{array}$} & $4(21 \%)$ & $23(34 \%)$ & $27(31 \%)$ \\
\hline \multicolumn{2}{|c|}{ High physical activity ( $\geq 3000$ MET minutes per week) } & $8(42 \%)$ & $15(22 \%)$ & $23(27 \%)$ \\
\hline \multicolumn{2}{|c|}{ Sedentary behaviour ( $\geq 3$ hours of sedentary time per day) } & $10(53 \%)$ & $32(48 \%)$ & $42(49 \%)$ \\
\hline \multicolumn{5}{|c|}{ Dietary Behaviour $(n=82)$} \\
\hline \multirow{2}{*}{$\begin{array}{l}\text { Fruits consumption } \\
(p=0.028)\end{array}$} & Never & 0 & $2(3 \%)$ & $2(2 \%)$ \\
\hline & $\begin{array}{l}\text { Once a week / less than once a } \\
\text { week }\end{array}$ & $4(21 \%)$ & $27(43 \%)$ & $31(28 \%)$ \\
\hline
\end{tabular}

MET = Metabolic equivalent of task; $n=$ number; $\%=$ percentage; $P A=$ physical activity;

p-value shown if significant at $5 \%$ level: $p<0.05$. 


\begin{tabular}{|c|c|c|c|c|}
\hline \multirow[t]{4}{*}{$\begin{array}{l}\text { Variable: Median (IQR) } \\
\text { or } n(\%)\end{array}$} & Description & $\begin{array}{l}\text { Male: } n= \\
19(22 \%)\end{array}$ & $\begin{array}{l}\text { Female: } n \\
=67(78 \%)\end{array}$ & $\begin{array}{l}\text { Total: } \mathrm{n}= \\
86\end{array}$ \\
\hline & 2-4 times a week & $5(26 \%)$ & $15(24 \%)$ & $20(24 \%)$ \\
\hline & Frequently (5-6 times a week) & $4(21 \%)$ & $2(3 \%)$ & $6(7 \%)$ \\
\hline & Daily or more than once daily & $6(32 \%)$ & $17(27 \%)$ & $23(2 \%)$ \\
\hline \multirow{5}{*}{$\begin{array}{l}\text { Vegetables } \\
\text { consumption }\end{array}$} & Never & 0 & $4(6 \%)$ & $4(5 \%)$ \\
\hline & $\begin{array}{l}\text { Once a week / less than once a } \\
\text { week }\end{array}$ & $3(17 \%)$ & $9(14 \%)$ & $12(15 \%)$ \\
\hline & 2-4 times a week & $1(6 \%)$ & $9(14 \%)$ & $10(12 \%)$ \\
\hline & Frequently (5-6 times a week) & $6(33 \%)$ & $7(11 \%)$ & $13(16 \%)$ \\
\hline & Daily or more than once daily & $8(44 \%)$ & $34(54 \%)$ & $42(52 \%)$ \\
\hline \multirow{5}{*}{$\begin{array}{l}\text { Wholegrain bread or } \\
\text { cereal Consumption } \\
(n=68)\end{array}$} & Never & $1(5 \%)$ & $4(6 \%)$ & $5(6 \%)$ \\
\hline & $\begin{array}{l}\text { Once a week / less than once a } \\
\text { week }\end{array}$ & $5(26 \%)$ & $16(25 \%)$ & $21(26 \%)$ \\
\hline & 2-4 times a week & $2(11 \%)$ & $6(10 \%)$ & $8(10 \%)$ \\
\hline & Frequently (5- 6 times a week) & $3(16 \%)$ & $4(6 \%)$ & $7(9 \%)$ \\
\hline & Daily or more than once daily & $5(26 \%)$ & $22(35 \%)$ & $27(33 \%)$ \\
\hline \multicolumn{2}{|c|}{ Daily consumption of sugar-sweetened beverages } & $5(26 \%)$ & $20(30 \%)$ & $25(29 \%)$ \\
\hline \multicolumn{2}{|c|}{ Daily consumption of deep-fried foods } & $3(16 \%)$ & $15(22 \%)$ & $18(21 \%)$ \\
\hline \multicolumn{2}{|c|}{ Daily consumption of fast foods } & $1(5 \%)$ & $12(18 \%)$ & $13(15 \%)$ \\
\hline \multicolumn{2}{|c|}{ Daily consumption of sweets \& cakes } & $2(11 \%)$ & $25(37 \%)$ & $27(31 \%)$ \\
\hline \multicolumn{2}{|c|}{ Ate a meal prepared outside the home in the past week } & $10(59 \%)$ & $45(73 \%)$ & $55(67 \%)$ \\
\hline & $\begin{array}{l}\text { Meals eaten outside the home in } \\
\text { past week: }(n=55)\end{array}$ & $2(1-10)$ & $2(2-3)$ & $2(2-4)$ \\
\hline \multicolumn{2}{|c|}{ Breakfast consumption: number of days in the past week } & $5(3-6)$ & $5(3-7)$ & $5(3-7)$ \\
\hline \multirow[t]{2}{*}{$\begin{array}{l}\text { Breakfast } \\
\text { consumption }\end{array}$} & $\begin{array}{l}\text { Skippers: ate breakfast 0-2 } \\
\text { days/week }\end{array}$ & $5(23 \%)$ & $13(20 \%)$ & $18(21 \%)$ \\
\hline & $\begin{array}{l}\text { Semi-skippers: ate breakfast 3-4 } \\
\text { days/week }\end{array}$ & $5(23 \%)$ & $13(20 \%)$ & $18(21 \%)$ \\
\hline
\end{tabular}

MET = Metabolic equivalent of task; $n=$ number $; \%=$ percentage; $P A=$ physical activity; p-value shown if significant at 5\% level: $p<0.05$. 


\begin{tabular}{|c|c|c|c|c|}
\hline $\begin{array}{l}\text { Variable: Median (IQR) } \\
\text { or } n(\%)\end{array}$ & Description & $\begin{array}{l}\text { Male: } n= \\
19(22 \%)\end{array}$ & $\begin{array}{l}\text { Female: } n \\
=67(78 \%)\end{array}$ & $\begin{array}{l}\text { Total: } n= \\
86\end{array}$ \\
\hline & $\begin{array}{l}\text { Non-skippers: ate breakfast 5-7 } \\
\text { days/week }\end{array}$ & $12(54 \%)$ & $39(60 \%)$ & $51(58 \%)$ \\
\hline \multicolumn{2}{|c|}{$\begin{array}{l}\text { Overall General Nutrition Knowledge score percentage \% } \\
\text { (score /88) }(95 \% \mathrm{Cl})\end{array}$} & $\begin{array}{l}40.3 \% \\
(34.5- \\
46.1)\end{array}$ & $\begin{array}{l}36.5 \% \\
(34.0- \\
38.9)\end{array}$ & $\begin{array}{l}37.3 \% \\
(35.1- \\
39.6)\end{array}$ \\
\hline & $\begin{array}{l}\text { 1. Dietary recommendations } \\
\text { (score/18) }\end{array}$ & $\begin{array}{l}44.2 \% \\
(35.3- \\
53.0)\end{array}$ & $\begin{array}{l}42.5 \% \\
(38.7- \\
46.3)\end{array}$ & $\begin{array}{l}42.9 \% \\
(39.4- \\
46.4)\end{array}$ \\
\hline & 2. Food Groups (score/36) & $\begin{array}{l}40.4 \\
(35.5- \\
45.4)\end{array}$ & $\begin{array}{l}37.1 \\
(34.1- \\
40.1)\end{array}$ & $\begin{array}{l}37.9 \\
(35.3- \\
40.4)\end{array}$ \\
\hline & $\begin{array}{l}\text { 3. Healthy food choices } \\
\text { (score/13) }\end{array}$ & $\begin{array}{l}36.8 \\
(27.3- \\
46.2)\end{array}$ & $\begin{array}{l}30.5 \\
(26.0- \\
35.1)\end{array}$ & $\begin{array}{l}31.9 \\
(27.9- \\
36.0)\end{array}$ \\
\hline & $\begin{array}{l}\text { 4. Diet, disease and weight } \\
\text { management (score/21) }\end{array}$ & $\begin{array}{l}36.8 \\
(28.2- \\
45.3)\end{array}$ & $\begin{array}{l}33.9 \\
(30.6- \\
37.1)\end{array}$ & $\begin{array}{l}34.5 \\
(31.4- \\
37.6)\end{array}$ \\
\hline \multicolumn{5}{|c|}{ MET = Metabolic equivalent of task; $n=$ number; $\%=$ percentage; $P A=$ physical activity; } \\
\hline \multicolumn{5}{|c|}{ p-value shown if significant at $5 \%$ level: $p<0.05$. } \\
\hline
\end{tabular}


Table 3

Physical Activity, Sedentary and Dietary Behaviour and Nutritional Knowledge by Age Group

\begin{tabular}{|c|c|c|c|c|c|c|}
\hline \multirow[t]{2}{*}{$\begin{array}{l}\text { Variable: Median } \\
\text { (IQR) or } n(\%)\end{array}$} & \multirow[t]{2}{*}{ Description } & $\begin{array}{l}15-17 \\
\text { years: }\end{array}$ & $\begin{array}{l}\text { 18-19 } \\
\text { years: }\end{array}$ & \multirow{2}{*}{$\begin{array}{l}20- \\
21 \text { years: } \\
n=23 \\
(27 \%)\end{array}$} & \multirow{2}{*}{$\begin{array}{l}22-24 \\
\text { years: } n \\
=29 \\
(34 \%)\end{array}$} & \multirow[t]{2}{*}{$\begin{array}{l}\text { Total: } \\
\mathrm{n}=86\end{array}$} \\
\hline & & $\begin{array}{l}n=11 \\
(13 \%)\end{array}$ & $\begin{array}{l}n=23 \\
(27 \%)\end{array}$ & & & \\
\hline \multirow[t]{3}{*}{$\begin{array}{l}\text { Vigorous intensity } \\
\text { Physical Activity }\end{array}$} & $\begin{array}{l}\text { Prevalence of PA for } \\
\geq 10 \mathrm{~min}\end{array}$ & $\begin{array}{l}8 \\
(80 \%)\end{array}$ & $\begin{array}{l}9 \\
(39 \%)\end{array}$ & $11(50 \%)$ & $9(31 \%)$ & $\begin{array}{l}37 \\
(43 \%)\end{array}$ \\
\hline & $\begin{array}{l}\text { Time spent per day in } \\
\text { minutes }\end{array}$ & $\begin{array}{l}120 \\
(30- \\
240)\end{array}$ & $\begin{array}{l}90 \\
(30- \\
120)\end{array}$ & $\begin{array}{l}105(30- \\
180)\end{array}$ & $\begin{array}{l}60(30- \\
60)\end{array}$ & $\begin{array}{l}60 \\
(30- \\
120)\end{array}$ \\
\hline & MET-minutes/week & $\begin{array}{l}4800 \\
(720- \\
9600)\end{array}$ & $\begin{array}{l}2160 \\
(1200- \\
2400)\end{array}$ & $\begin{array}{l}2520 \\
(960- \\
4320)\end{array}$ & $\begin{array}{l}1440 \\
(480- \\
1920)\end{array}$ & $\begin{array}{l}1920 \\
(720- \\
4320)\end{array}$ \\
\hline \multirow[t]{3}{*}{$\begin{array}{l}\text { Moderate-intensity } \\
\text { Physical Activity }\end{array}$} & $\begin{array}{l}\text { Prevalence of PA for } \\
\geq 10 \text { minutes }\end{array}$ & $\begin{array}{l}5 \\
(50 \%)\end{array}$ & $\begin{array}{l}17 \\
(74 \%)\end{array}$ & 19 (86\%) & $25(86 \%)$ & $\begin{array}{l}68 \\
(79 \%)\end{array}$ \\
\hline & $\begin{array}{l}\text { Time spent per day in } \\
\text { mins }\end{array}$ & $\begin{array}{l}60 \\
(30- \\
60)\end{array}$ & $\begin{array}{l}60 \\
(40- \\
120)\end{array}$ & $\begin{array}{l}30(30- \\
120)\end{array}$ & $\begin{array}{l}30(30- \\
60)\end{array}$ & $\begin{array}{l}60 \\
(30- \\
90)\end{array}$ \\
\hline & MET-minutes/week & $\begin{array}{l}720 \\
(360- \\
720)\end{array}$ & $\begin{array}{l}1440 \\
(640- \\
1680)\end{array}$ & $\begin{array}{l}600 \\
(420- \\
1680)\end{array}$ & $\begin{array}{l}600 \\
(480- \\
840)\end{array}$ & $\begin{array}{l}720 \\
(480- \\
1440)\end{array}$ \\
\hline \multirow{3}{*}{$\begin{array}{l}\text { Active Travel } \\
\text { Walking or cycling }\end{array}$} & $\begin{array}{l}\text { Walking or cycling } \\
\text { for } \geq 10 \text { minutes }\end{array}$ & $\begin{array}{l}7 \\
(70 \%)\end{array}$ & $\begin{array}{l}19 \\
(83 \%)\end{array}$ & $14(64 \%)$ & $20(69 \%)$ & $\begin{array}{l}60 \\
(71 \%)\end{array}$ \\
\hline & $\begin{array}{l}\text { Time spent daily in } \\
\text { mins }\end{array}$ & $\begin{array}{l}30 \\
(30- \\
120)\end{array}$ & $\begin{array}{l}30 \\
(30- \\
60)\end{array}$ & $\begin{array}{l}60(30- \\
60)\end{array}$ & $\begin{array}{l}30(30- \\
60)\end{array}$ & $\begin{array}{l}35 \\
(30- \\
60)\end{array}$ \\
\hline & $\begin{array}{l}\text { Walking MET- } \\
\text { minutes/week }\end{array}$ & $\begin{array}{l}495 \\
(495- \\
495)\end{array}$ & $\begin{array}{l}693 \\
(396- \\
1386)\end{array}$ & $\begin{array}{l}742.5 \\
(495- \\
1188)\end{array}$ & $\begin{array}{l}495 \\
(198- \\
693)\end{array}$ & $\begin{array}{l}528 \\
(297- \\
990)\end{array}$ \\
\hline \multicolumn{2}{|c|}{ Total physical activity MET-minutes/week } & $\begin{array}{l}984 \\
(280- \\
5295)\end{array}$ & $\begin{array}{l}2160 \\
(800- \\
3756)\end{array}$ & $\begin{array}{l}1638.75 \\
(420- \\
6624)\end{array}$ & $\begin{array}{l}876 \\
(508.5- \\
1878)\end{array}$ & $\begin{array}{l}1215 \\
(495- \\
3348)\end{array}$ \\
\hline \multicolumn{2}{|c|}{$\begin{array}{l}\text { Insufficient physical activity (< } 600 \text { MET-mins } \\
\text { per week) }\end{array}$} & $\begin{array}{l}5 \\
(45 \%)\end{array}$ & $\begin{array}{l}5 \\
(22 \%)\end{array}$ & $7(30 \%)$ & $10(34 \%)$ & $\begin{array}{l}27 \\
(31 \%)\end{array}$ \\
\hline \multicolumn{2}{|c|}{$\begin{array}{l}\text { High physical activity ( } \geq 3000 \text { MET mins per } \\
\text { week) }\end{array}$} & $\begin{array}{l}3 \\
(27 \%)\end{array}$ & $\begin{array}{l}8 \\
(35 \%)\end{array}$ & $9(39 \%)$ & $3(10 \%)$ & $\begin{array}{l}23 \\
(27 \%)\end{array}$ \\
\hline
\end{tabular}

$n=$ number $; \%$ = percentage; mins = minutes; $P A=$ physical activity; $M E T=$ Metabolic equivalent of task;

p-value from Kruskal Wallis test shown if significant at $5 \%$ level: $p<0.05$. 


\begin{tabular}{|c|c|c|c|c|c|c|}
\hline $\begin{array}{l}\text { Variable: Median } \\
\text { (IQR) or } n(\%)\end{array}$ & Description & $\begin{array}{l}15-17 \\
\text { years: } \\
n=11 \\
(13 \%)\end{array}$ & $\begin{array}{l}18-19 \\
\text { years: } \\
n=23 \\
(27 \%)\end{array}$ & $\begin{array}{l}20- \\
21 \text { years: } \\
n=23 \\
(27 \%)\end{array}$ & $\begin{array}{l}22-24 \\
\text { years: } n \\
=29 \\
(34 \%)\end{array}$ & $\begin{array}{l}\text { Total: } \\
\mathrm{n}=86\end{array}$ \\
\hline \multicolumn{2}{|c|}{$\begin{array}{l}\text { Sedentary behaviour ( } \geq 3 \text { hours of sedentary } \\
\text { time per day) }\end{array}$} & $\begin{array}{l}7 \\
(70 \%)\end{array}$ & $\begin{array}{l}9 \\
(39 \%)\end{array}$ & $12(54 \%)$ & $14(49 \%)$ & $\begin{array}{l}42 \\
(49 \%)\end{array}$ \\
\hline \multicolumn{7}{|c|}{ Dietary Behaviour $(n=82)$} \\
\hline \multirow[t]{5}{*}{ Fruits consumption } & Never & 0 & 0 & 0 & $2(7 \%)$ & $2(2 \%)$ \\
\hline & $\begin{array}{l}\text { Once a week / less } \\
\text { than once a week }\end{array}$ & $\begin{array}{l}5 \\
(50 \%)\end{array}$ & $\stackrel{9}{(39 \%)}$ & $10(45 \%)$ & $7(26 \%)$ & $\begin{array}{l}31 \\
(38 \%)\end{array}$ \\
\hline & 2-4 times a week & $\begin{array}{l}2 \\
(20 \%)\end{array}$ & $\begin{array}{l}5 \\
(22 \%)\end{array}$ & $4(18 \%)$ & $9(33 \%)$ & $\begin{array}{l}20 \\
(24 \%)\end{array}$ \\
\hline & $\begin{array}{l}\text { Frequently (5-6 } \\
\text { times a week) }\end{array}$ & $\begin{array}{l}2 \\
(20 \%)\end{array}$ & 0 & $3(14 \%)$ & $1(4 \%)$ & $6(7 \%)$ \\
\hline & $\begin{array}{l}\text { Daily or more than } \\
\text { once daily }\end{array}$ & $\begin{array}{l}1 \\
(10 \%)\end{array}$ & $\begin{array}{l}9 \\
(39 \%)\end{array}$ & $5(23 \%)$ & $8(30 \%)$ & $\begin{array}{l}23 \\
(28 \%)\end{array}$ \\
\hline \multirow{5}{*}{$\begin{array}{l}\text { Vegetables } \\
\text { consumption }\end{array}$} & Never & 0 & $1(4 \%)$ & $1(5 \%)$ & $2(7 \%)$ & $4(5 \%)$ \\
\hline & $\begin{array}{l}\text { Once a week / less } \\
\text { than once a week }\end{array}$ & $\begin{array}{l}2 \\
(20 \%)\end{array}$ & $\begin{array}{l}5 \\
(22 \%)\end{array}$ & $3(14 \%)$ & $2(7 \%)$ & $\begin{array}{l}12 \\
(15 \%)\end{array}$ \\
\hline & 2-4 times a week & $\begin{array}{l}2 \\
(20 \%)\end{array}$ & $1(4 \%)$ & 0 & $7(265)$ & $\begin{array}{l}10 \\
(12 \%)\end{array}$ \\
\hline & $\begin{array}{l}\text { Frequently }(5-6 \\
\text { times a week) }\end{array}$ & $\begin{array}{l}2 \\
(20 \%)\end{array}$ & $\begin{array}{l}4 \\
(17 \%)\end{array}$ & $6(29 \%)$ & $1(4 \%)$ & $\begin{array}{l}13 \\
(16 \%)\end{array}$ \\
\hline & $\begin{array}{l}\text { Daily or more than } \\
\text { once daily }\end{array}$ & $\begin{array}{l}4 \\
(40 \%)\end{array}$ & $\begin{array}{l}12 \\
(52 \%)\end{array}$ & $11(52 \%)$ & $15(56 \%)$ & $\begin{array}{l}42 \\
(52 \%)\end{array}$ \\
\hline \multirow{5}{*}{$\begin{array}{l}\text { Whole-grain bread } \\
\text { or cereal } \\
\text { consumption }(n= \\
68)\end{array}$} & Never & 0 & $1(4 \%)$ & $1(5 \%)$ & $3(11 \%)$ & $5(6 \%)$ \\
\hline & $\begin{array}{l}\text { Once a week / less } \\
\text { than once a week }\end{array}$ & $\begin{array}{l}5 \\
(50 \%)\end{array}$ & $\begin{array}{l}7 \\
(30 \%)\end{array}$ & $5(23 \%)$ & $4(15 \%)$ & $\begin{array}{l}21 \\
(26 \%)\end{array}$ \\
\hline & 2-4 times a week & $\begin{array}{l}2 \\
(20 \%)\end{array}$ & $1(4 \%)$ & $1(5 \%)$ & $4(15 \%)$ & $8)$ \\
\hline & $\begin{array}{l}\text { Frequently }(5-6 \\
\text { times a week) }\end{array}$ & $\begin{array}{l}1 \\
(10 \%)\end{array}$ & $\begin{array}{l}4 \\
(17 \%)\end{array}$ & $1(5 \%)$ & $1(4 \%)$ & $7(9 \%)$ \\
\hline & $\begin{array}{l}\text { Daily or more than } \\
\text { once daily }\end{array}$ & $\begin{array}{l}1 \\
(10 \%)\end{array}$ & $\stackrel{9}{(39 \%)}$ & $7(32 \%)$ & $10(37 \%)$ & $\begin{array}{l}27 \\
(33 \%)\end{array}$ \\
\hline
\end{tabular}

$n=$ number $; \%=$ percentage $;$ mins = minutes; $P A=$ physical activity; $M E T=$ Metabolic equivalent of task;

p-value from Kruskal Wallis test shown if significant at $5 \%$ level: $p<0.05$. 


\begin{tabular}{|c|c|c|c|c|c|c|}
\hline $\begin{array}{l}\text { Variable: Median } \\
\text { (IQR) or } n(\%)\end{array}$ & Description & $\begin{array}{l}15-17 \\
\text { years: } \\
n=11 \\
(13 \%)\end{array}$ & $\begin{array}{l}18-19 \\
\text { years: } \\
n=23 \\
(27 \%)\end{array}$ & $\begin{array}{l}20- \\
21 \text { years: } \\
n=23 \\
(27 \%)\end{array}$ & $\begin{array}{l}22-24 \\
\text { years: } n \\
=29 \\
(34 \%)\end{array}$ & $\begin{array}{l}\text { Total: } \\
\mathrm{n}=86\end{array}$ \\
\hline \multicolumn{2}{|c|}{$\begin{array}{l}\text { Daily consumption of sugar-sweetened } \\
\text { beverages }\end{array}$} & $\begin{array}{l}3 \\
(27 \%)\end{array}$ & $\begin{array}{l}6 \\
(26 \%)\end{array}$ & $9(39 \%)$ & $7(24 \%)$ & $\begin{array}{l}25 \\
(29 \%)\end{array}$ \\
\hline \multicolumn{2}{|c|}{$\begin{array}{l}\text { Daily consumption of deep-fried foods ( } p= \\
0.031 \text { ) }\end{array}$} & $1(9 \%)$ & $\begin{array}{l}8 \\
(35 \%)\end{array}$ & $5(22 \%)$ & $4(14 \%)$ & $\begin{array}{l}18 \\
(21 \%)\end{array}$ \\
\hline \multicolumn{2}{|c|}{ Daily consumption of fast foods } & $\begin{array}{l}2 \\
(18 \%)\end{array}$ & $\begin{array}{l}6 \\
(26 \%)\end{array}$ & $2(9 \%)$ & $3(10 \%)$ & $\begin{array}{l}13 \\
(15 \%)\end{array}$ \\
\hline \multicolumn{2}{|c|}{ Daily consumption of sweets \& cakes } & $\begin{array}{l}4 \\
(36 \%)\end{array}$ & $\begin{array}{l}9 \\
(39 \%)\end{array}$ & $6(26 \%)$ & $8(28 \%)$ & $\begin{array}{l}27 \\
(31 \%)\end{array}$ \\
\hline \multicolumn{7}{|c|}{ Dietary Behaviour $(n=82)$} \\
\hline \multicolumn{2}{|c|}{$\begin{array}{l}\text { Ate meal prepared outside the home in past } \\
\text { week }\end{array}$} & $\begin{array}{l}5 \\
(50 \%)\end{array}$ & $\begin{array}{l}14 \\
(64 \%)\end{array}$ & $15(68 \%)$ & $21(78 \%)$ & $\begin{array}{l}55 \\
(67 \%)\end{array}$ \\
\hline & $\begin{array}{l}\text { Meals eaten outside } \\
\text { the home in past } \\
\text { week: }(n=55)\end{array}$ & $\begin{array}{l}2(1- \\
3)\end{array}$ & $\begin{array}{l}2(2- \\
4)\end{array}$ & $2(2-4)$ & $2(2-3)$ & $\begin{array}{l}2(2- \\
4)\end{array}$ \\
\hline \multirow[t]{3}{*}{$\begin{array}{l}\text { Breakfast } \\
\text { consumption }\end{array}$} & $\begin{array}{l}\text { Skippers: ate } \\
\text { breakfast 0-2 } \\
\text { days/week }\end{array}$ & $\begin{array}{l}2 \\
(20 \%)\end{array}$ & $\begin{array}{l}7 \\
(30 \%)\end{array}$ & $3(14 \%)$ & $7(26 \%)$ & $\begin{array}{l}19 \\
(23 \%)\end{array}$ \\
\hline & $\begin{array}{l}\text { Semi-skippers: ate } \\
\text { breakfast 3-4 } \\
\text { days/week }\end{array}$ & $\begin{array}{l}1 \\
(10 \%)\end{array}$ & $\begin{array}{l}4 \\
(17 \%)\end{array}$ & $6(27 \%)$ & $3(11 \%)$ & $\begin{array}{l}14 \\
(17 \%)\end{array}$ \\
\hline & $\begin{array}{l}\text { Non-skippers: ate } \\
\text { breakfast 5-7 } \\
\text { days/week }\end{array}$ & $\begin{array}{l}7 \\
(70 \%)\end{array}$ & $\begin{array}{l}12 \\
(52 \%)\end{array}$ & $13(59 \%)$ & $17(63 \%)$ & $\begin{array}{l}49 \\
(60 \%)\end{array}$ \\
\hline \multicolumn{2}{|c|}{$\begin{array}{l}\text { Overall General Nutrition Knowledge score } \\
\text { percentage } \\
(\text { score } / 88) \text { mean }(95 \% \mathrm{Cl})\end{array}$} & $\begin{array}{l}35.6 \\
(28.4- \\
42.9)\end{array}$ & $\begin{array}{l}38.1 \\
(34.1- \\
42.1)\end{array}$ & $\begin{array}{l}34.3 \\
(28.9- \\
39.6)\end{array}$ & $\begin{array}{l}39.6 \\
(35.7- \\
43.4)\end{array}$ & $\begin{array}{l}37.3 \\
(35.1- \\
39.6)\end{array}$ \\
\hline & $\begin{array}{l}\text { 1. Dietary } \\
\text { recommendations } \\
\text { (score/18) }\end{array}$ & $\begin{array}{l}39.9 \\
(27.8- \\
52.0)\end{array}$ & $\begin{array}{l}46.1 \\
(39.8- \\
52.4)\end{array}$ & $\begin{array}{l}40.2 \\
(32.3- \\
48.1)\end{array}$ & $\begin{array}{l}43.4 \\
(37.5- \\
49.3)\end{array}$ & $\begin{array}{l}42.9 \\
(39.4- \\
46.4)\end{array}$ \\
\hline & $\begin{array}{l}\text { 2. Food Groups } \\
\text { (score/36) }\end{array}$ & $\begin{array}{l}37.6 \\
(31.6- \\
43.7)\end{array}$ & $\begin{array}{l}37.4 \\
(32.3- \\
42.5)\end{array}$ & $\begin{array}{l}35.0 \\
(29.7- \\
40.3)\end{array}$ & $\begin{array}{l}40.4 \\
(35.5- \\
45.4)\end{array}$ & $\begin{array}{l}37.9 \\
(35.3- \\
40.4)\end{array}$ \\
\hline
\end{tabular}

$n=$ number $; \%$ = percentage; mins = minutes; $P A=$ physical activity; $M E T=$ Metabolic equivalent of task;

p-value from Kruskal Wallis test shown if significant at $5 \%$ level: $p<0.05$. 


\begin{tabular}{|c|c|c|c|c|c|c|}
\hline \multirow[t]{3}{*}{$\begin{array}{l}\text { Variable: Median } \\
\text { (IQR) or } \mathrm{n}(\%)\end{array}$} & Description & $\begin{array}{l}15-17 \\
\text { years: } \\
n=11 \\
(13 \%)\end{array}$ & $\begin{array}{l}18-19 \\
\text { years: } \\
n=23 \\
(27 \%)\end{array}$ & $\begin{array}{l}20- \\
21 \text { years: } \\
n=23 \\
(27 \%)\end{array}$ & $\begin{array}{l}22-24 \\
\text { years: } n \\
=29 \\
(34 \%)\end{array}$ & $\begin{array}{l}\text { Total: } \\
\mathrm{n}=86\end{array}$ \\
\hline & $\begin{array}{l}\text { 3. Healthy Food } \\
\text { choices (score/13) }\end{array}$ & $\begin{array}{l}33.6 \\
(21.5- \\
45.6)\end{array}$ & $\begin{array}{l}35.1 \\
(28.4- \\
41.8)\end{array}$ & $\begin{array}{l}27.7 \\
(17.8- \\
37.6)\end{array}$ & $\begin{array}{l}31.6 \\
(24.2- \\
39.0)\end{array}$ & $\begin{array}{l}31.9 \\
(27.9- \\
36.0)\end{array}$ \\
\hline & $\begin{array}{l}\text { 4. Diet, disease } \\
\text { relationship } \\
\text { (score/21) }\end{array}$ & $\begin{array}{l}29.9 \\
(20.6- \\
39.2)\end{array}$ & $\begin{array}{l}34.4 \\
(29.4- \\
39.3)\end{array}$ & $\begin{array}{l}30.2 \\
(23.3- \\
37.1)\end{array}$ & $\begin{array}{l}39.7 \\
(34.0- \\
45.3)\end{array}$ & $\begin{array}{l}34.5 \\
(31.4- \\
37.6)\end{array}$ \\
\hline \multicolumn{7}{|c|}{$\begin{array}{l}n=\text { number; } \%=\text { percentage; } \text { mins = minutes; } P A=\text { physical activity; } M E T=\text { Metabolic equivalent of } \\
\text { task; }\end{array}$} \\
\hline \multicolumn{7}{|c|}{ p-value from Kruskal Wallis test shown if significant at $5 \%$ level: $p<0.05$. } \\
\hline
\end{tabular}

\section{Dietary intake}

Overall, less than a third of respondents ate fruits and wholegrains daily (Table 2). Significantly more males ate fruit frequently compared to females (53\% versus $28 \%$ ). More than half ate vegetables daily (either dark green, orange or other vegetables). Younger adolescents had the lowest daily consumption of fruits, vegetables and wholegrains compared to older age groups. A third of respondents had a high dietary intake of sugar; with 29\% reporting drinking SSB daily (including regular soft drinks, energy drinks and sports drinks) and 31\% eating sweets and cakes daily. SSB consumption was similar across gender and age groups. One in five participants ate deep-fried foods daily and $15 \%$ ate fast-foods daily. Older adolescents aged 18-19 years had the highest daily consumption of deep-fried (35\%) and fast foods (26\%) (See Table 3).

Meals eaten outside the home

Two-thirds of respondents ate at least one meal that was prepared outside the home in the previous week. Those who ate food prepared outside the home, ate a median of two take-away or sit-down meals in the past week (IQR 2-4 meals). Males had a larger variability in meals consumed that were prepared outside the home compared to females; median 2 meals (IQR 1-10 meals) versus (IQR 2-3 meals) respectively.

\section{Breakfast}

Over half of participants (58\%) reported eating breakfast on at least five days in the previous week, $21 \%$ ate breakfast on 3-4 days in the week and $21 \%$ reported skipping breakfast or eating breakfast on less than two days in the previous week. Breakfast skipping did not differ significantly by gender or age.

\section{Nutrition Knowledge}


Overall, the mean general nutrition knowledge score achieved by adolescents was 33 out of a total of 88 points (37.5\%). Knowledge of dietary recommendations was the highest-scoring domain with an average of 7.9 points out of $18(44 \%)$ while knowledge of healthy food choices was the lowest-scoring domain (average of 4.2 out of 13 points; (32\%)). Participants scored an average of 13.45 points out of 36 for knowledge of food groups (37\%); and 7.3/21 points for knowledge of diet-disease relationships (35\%). There were no significant differences in nutrition knowledge amongst adolescents by sex and age group (See Fig. 2).

\section{Tobacco use and exposure}

Significantly more males (58\%) than females (30\%) reported ever smoking cigarettes (Table 4). The median age when participants first smoked cigarettes was 16 years (IQR 15-17 years). Overall, 30\% had smoked cigarettes in the past month: $9 \%$ reported smoking daily while $21 \%$ smoked less often than daily (37\% males and $16 \%$ females). The oldest age group (22-24 years) reported the highest rates of ever smoking (41\%) while the prevalence of daily smoking was highest for those aged $18-19$ years $(13 \%)$. In the youngest age group of 15-17-year olds, $45 \%$ reported smoking cigarettes (less often than daily) in the past month (see Fig. 3). Overall, $21 \%$ reported using tobacco products other than cigarettes (including water or hookah pipes and vaping) during the past month, with $6 \%$ reporting using these products on six or more days in the past month. Those in the youngest age group reported the most use of tobacco products other than cigarettes in the past month; $45 \%$ compared to less than $30 \%$ in the other age groups (Table 5). Almost half the participants (45\%) reported being exposed to secondary smoke from people who smoked in their presence in the past week. More than half $(15 / 26)$ of current smokers also drank alcohol in the preceding month. 
Table 4

Tobacco Use and Exposure, Alcohol and Substance use by Gender

\begin{tabular}{|c|c|c|c|}
\hline able: Median (IQR) or n (\%) & Description & $\begin{array}{l}\text { Male: } n= \\
19(22 \%)\end{array}$ & $\begin{array}{l}\text { Female: } n \\
=67(78 \%)\end{array}$ \\
\hline
\end{tabular}

Tobacco and Alcohol Use

Ever smoked cigarettes in lifetime $(p=0.025)$

$11(58 \%) \quad 20(30 \%) \quad 31$

$(36 \%)$

Age when first smoked cigarettes

$\begin{array}{lll}15(13- & 16(15- & 16 \\ 16) & 17) & (15- \\ & & 17)\end{array}$

Days smoked during the past 30 days

0 days

$10(53 \%) \quad 50(75 \%)$

60

$(70 \%)$

1 or 2 days

$3(16 \%) \quad 8(12 \%)$

11

3 to 5 days

$4(21 \%)$

$2(3 \%)$

$6(7 \%)$

20 to 29 days

0

$1(1 \%)$

$1(1 \%)$

Daily: all 30 days

$2(11 \%)$

$6(9 \%)$

$8(9 \%)$

Days used any tobacco products other

than cigarettes, during past month

0 days

$13(68 \%)$

$55(82 \%)$

$(p=0.055)$

1 or 2 days

$3(16 \%)$

$8(12 \%)$

11

3 to 5 days

$2(11 \%)$

0

$2(2 \%)$

6 or more days

$1(5 \%)$

$4(6 \%)$

$5(6 \%)$

People smoked around you during past 7 days

$10(53 \%) \quad 29(43 \%)$

39

Days people smoked in their presence during the past 7 days

\begin{tabular}{|llll}
\hline 0 days & $4(21 \%)$ & $20(30 \%)$ & $\begin{array}{l}24 \\
(28 \%)\end{array}$ \\
\hline 1 or 2 days & $4(21 \%)$ & $25(37 \%)$ & $\begin{array}{l}29 \\
(34 \%)\end{array}$ \\
\hline 3 or 4 days & $6(32 \%)$ & $8(12 \%)$ & $\begin{array}{l}14 \\
(16 \%)\end{array}$ \\
\hline 5 or 6 days & $2(10 \%)$ & $5(7 \%)$ & $7(8 \%)$
\end{tabular}

${ }^{1}$ Inhalants = glue, aerosols, paint thinners, petrol or benzene;

${ }^{2}$ OTC $=$ over-the-counter;

p-value shown if significant at $10 \%$ level: $p<0.10$. 
Variable: Median (IQR) or $\mathrm{n}(\%)$

Ever drunk alcohol $(n=85)$
All 7 days
Description

\begin{tabular}{llll} 
& $13(68 \%)$ & $45(67 \%)$ & $\begin{array}{l}58 \\
(67 \%)\end{array}$ \\
$\begin{array}{llll}\text { Age at alcohol } \\
\text { initiation in years }\end{array}$ & $\begin{array}{l}16(14- \\
19)\end{array}$ & $\begin{array}{l}17(15- \\
18)\end{array}$ & $\begin{array}{l}16.5 \\
(15- \\
18)\end{array}$ \\
\hline $\begin{array}{l}\text { Drunk alcohol in } \\
\text { the past 30 days }\end{array}$ & $11(58 \%)$ & $24(36 \%)$ & $\begin{array}{l}35 \\
(41 \%)\end{array}$ \\
\hline $\begin{array}{l}\text { Binge drinking in } \\
\text { past 30 days }\end{array}$ & $7(37 \%)$ & $14(21 \%)$ & $\begin{array}{l}21 \\
(24 \%)\end{array}$
\end{tabular}

Illegal and Other Drug Use

Ever used cannabis (dagga) $(p=0.087)$

$8(42 \%)$

$15(22 \%)$

23

(27\%)

Age at first

cannabis use

$16(13-$

19)

$16(15-$

18)

16

(14.5-

18.5)

Cannabis use in the past year

$6(32 \%) \quad 13(19 \%)$

19

Frequency of dagga use in past year

Almost every day

$2(11 \%)$

$3(4 \%)$

5 (6\%)

Once a week or more

$1(5 \%)$

$3(4 \%)$

$4(5 \%)$

About once a month

$1(5 \%)$

$5(7 \%)$

$6(7 \%)$

Only once or twice

$2(11 \%) \quad 2(3 \%)$

$4(5 \%)$

Never

$13(68 \%)$

$54(81 \%)$

67

(78\%)

Ever taken cocaine, heroin, mandrax, club drugs or methamphetamine

$1(5 \%)$

$18(95 \%) \quad 62(93 \%)$

80

Lifetime use of cocaine, heroin, mandrax, 0 times club drugs or methamphetamine

$5(7 \%)$

$6(7 \%)$

$(93 \%)$

${ }^{1}$ Inhalants = glue, aerosols, paint thinners, petrol or benzene;

${ }^{2}$ OTC = over-the-counter;

p-value shown if significant at $10 \%$ level: $p<0.10$. 


\begin{tabular}{|c|c|c|c|c|}
\hline \multirow[t]{4}{*}{ Variable: Median (IQR) or $\mathrm{n}(\%)$} & Description & $\begin{array}{l}\text { Male: } n= \\
19(22 \%)\end{array}$ & $\begin{array}{l}\text { Female: } n \\
=67(78 \%)\end{array}$ & $\begin{array}{l}\text { Total: } \\
\mathrm{n}=86\end{array}$ \\
\hline & 1 or 2 times & 0 & $2(3 \%)$ & $2(2 \%)$ \\
\hline & 3 to 9 times & 0 & $1(1 \%)$ & $1(1 \%)$ \\
\hline & 20 or more times & $1(5 \%)$ & $2(3 \%)$ & $3(3 \%)$ \\
\hline \multicolumn{2}{|l|}{ Ever used inhalants ${ }^{1}$ "to get high" } & $4(21 \%)$ & $5(7 \%)$ & $\begin{array}{l}9 \\
(10 \%)\end{array}$ \\
\hline \multirow[t]{3}{*}{ Lifetime use of inhalants to get high } & 0 times & $16(84 \%)$ & $64(96 \%)$ & $\begin{array}{l}80 \\
(93 \%)\end{array}$ \\
\hline & 1 or 2 times & $2(11 \%)$ & $3(4 \%)$ & $5(6 \%)$ \\
\hline & 3 to 9 times & $1(5 \%)$ & 0 & $1(1 \%)$ \\
\hline \multicolumn{2}{|c|}{ Ever used $\mathrm{OTC}^{2}$ or prescription drugs "to get high" } & $5(26 \%)$ & $8(12 \%)$ & $\begin{array}{l}13 \\
(15 \%)\end{array}$ \\
\hline \multirow[t]{3}{*}{ Lifetime use of OTC to "to get high" } & 0 times & $15(79 \%)$ & $61(91 \%$ & $\begin{array}{l}76 \\
(88 \%)\end{array}$ \\
\hline & 1 or 2 times & $3(16 \%)$ & $6(9 \%)$ & $\begin{array}{l}9 \\
(10 \%)\end{array}$ \\
\hline & 3 to 9 times & $1(5 \%)$ & 0 & $1(1 \%)$ \\
\hline \multicolumn{2}{|c|}{$\begin{array}{l}\text { Ever used any illegal drugs or substance "to get high" ( } p= \\
0.088)\end{array}$} & $10(53 \%)$ & $21(31 \%)$ & $\begin{array}{l}31 \\
(36 \%)\end{array}$ \\
\hline \multicolumn{5}{|c|}{${ }^{1}$ Inhalants = glue, aerosols, paint thinners, petrol or benzene; } \\
\hline \multicolumn{5}{|l|}{${ }^{2}$ OTC $=$ over-the-counter; } \\
\hline \multicolumn{5}{|c|}{$p$-value shown if significant at $10 \%$ level: $p<0.10$. } \\
\hline
\end{tabular}


Table 5

Tobacco Use and Exposure, Alcohol and Substance use by Age Group

\begin{tabular}{|c|c|c|c|c|c|c|}
\hline Variable: Median (IQR) or $n(\%)$ & Description & $\begin{array}{l}15-17 \\
\text { years: } \\
n=11 \\
(13 \%)\end{array}$ & $\begin{array}{l}18- \\
19 \\
\text { years: } \\
n=23 \\
(27 \%)\end{array}$ & $\begin{array}{l}20-21 \\
\text { years: } n \\
=23 \\
(27 \%)\end{array}$ & $\begin{array}{l}22- \\
24 \\
\text { years: } \\
\mathrm{n}=29 \\
(34 \%)\end{array}$ & $\begin{array}{l}\text { Total: } \\
\mathrm{n}=86\end{array}$ \\
\hline
\end{tabular}

Tobacco and Alcohol Use

Ever smoked cigarettes in lifetime

$\begin{array}{lllll}5 & 7 & 7(30 \%) & 12 & 31 \\ (45 \%) & (30 \%) & & (41 \%) & (36 \%) \\ 16 & 15.5 & 15(14- & 17 & 16 \\ (13- & (13- & 16) & (16- & (15- \\ 16) & 16) & & 20) & 17)\end{array}$

Days smoked during the past $30 \quad 0$ days days

Age when first smoked cigarettes $(p=0.0686)$

0 days $\quad 6$

6

1 or 2 days

5

3 to 5 days
20 to 29
days

16

(70\%)

$17(74 \%) \quad 21$

$(72 \%)$

60

$(70 \%)$

$20 \%$

All 30 days 0

Days used any tobacco products 0 days other than cigarettes, during past month

0 days

1 or 2 days

$\begin{array}{ll}6 & 19 \\ (55 \%) & (83 \%)\end{array}$

$1(4 \%)$

$\begin{array}{ll}3 & 11 \\ (10 \%) & (13 \%)\end{array}$

1

$14 \%$

$3(13 \%) \quad 2 \quad(7 \%)$

$6(7 \%)$

0

\begin{tabular}{|c|c|c|}
\hline $\begin{array}{l}3 \\
(13 \%)\end{array}$ & $2(9 \%)$ & $\begin{array}{l}3 \\
(10 \%)\end{array}$ \\
\hline
\end{tabular}

$\begin{array}{llllll}3 \text { to } 5 \text { days } & 0 & 0 & 2(9 \%) & 0 & 2(2 \%) \\ \begin{array}{l}\begin{array}{l}6 \text { or more } \\ \text { days }\end{array} \\ \text { days }\end{array} & \begin{array}{l}1 \\ (4 \%)\end{array} & 2(9 \%) & \begin{array}{l}1 \\ (3 \%)\end{array} & 5(6 \%)\end{array}$

$\begin{array}{llllll}\text { People smoked around you during past } 7 \text { days } & 5 & 11 & 10(43 \%) & 13 & 39 \\ & (45 \%) & (48 \%) & & (45 \%) & (45 \%)\end{array}$

${ }^{1} P$-value associated with Kruskal-Wallis equality-of-populations rank test;

${ }^{2}$ Fisher's exact $p$-value;

${ }^{3}$ Inhalants = glue, aerosols, paint thinners, petrol or benzene;

${ }^{4}$ OTC $=$ over-the-counter. 


\begin{tabular}{|c|c|c|c|c|c|c|}
\hline Variable: Median (IQR) or $\mathrm{n}(\%)$ & Description & $\begin{array}{l}15-17 \\
\text { years: } \\
n=11 \\
(13 \%)\end{array}$ & $\begin{array}{l}18- \\
19 \\
\text { years: } \\
n=23 \\
(27 \%)\end{array}$ & $\begin{array}{l}20-21 \\
\text { years: } n \\
=23 \\
(27 \%)\end{array}$ & $\begin{array}{l}22- \\
24 \\
\text { years: } \\
n=29 \\
(34 \%)\end{array}$ & $\begin{array}{l}\text { Total: } \\
\mathrm{n}=86\end{array}$ \\
\hline \multirow[t]{5}{*}{$\begin{array}{l}\text { Days people smoked in their } \\
\text { presence during the past } 7 \text { days }\end{array}$} & 0 days & $1(9 \%)$ & $\begin{array}{l}7 \\
(30 \%)\end{array}$ & $5(22 \%)$ & $\begin{array}{l}11 \\
(38 \%)\end{array}$ & $\begin{array}{l}24 \\
(28 \%)\end{array}$ \\
\hline & 1 or 2 days & $\begin{array}{l}5 \\
(45 \%)\end{array}$ & $\begin{array}{l}9 \\
(39 \%)\end{array}$ & $8(35 \%)$ & $\begin{array}{l}7 \\
(24 \%)\end{array}$ & $\begin{array}{l}29 \\
(34 \%)\end{array}$ \\
\hline & 3 or 4 days & $\begin{array}{l}2 \\
(18 \%)\end{array}$ & 0 & $5(22 \%)$ & $\begin{array}{l}7 \\
(24 \%)\end{array}$ & $\begin{array}{l}14 \\
(16 \%)\end{array}$ \\
\hline & 5 or 6 days & $\begin{array}{l}2 \\
(18 \%)\end{array}$ & $\begin{array}{l}1 \\
(4 \%)\end{array}$ & $3(13 \%)$ & $13 \%)$ & $7(8 \%)$ \\
\hline & All 7 days & $1(9 \%)$ & $\begin{array}{l}6 \\
(26 \%)\end{array}$ & $2(9 \%)$ & $\begin{array}{l}3 \\
(10 \%)\end{array}$ & $\begin{array}{l}12 \\
(14 \%)\end{array}$ \\
\hline \multicolumn{2}{|l|}{ Ever drunk alcohol } & $\begin{array}{l}9 \\
(82 \%)\end{array}$ & $\begin{array}{l}14 \\
(61 \%)\end{array}$ & $14(61 \%)$ & $\begin{array}{l}21 \\
(75 \%)\end{array}$ & $\begin{array}{l}58 \\
(68 \%)\end{array}$ \\
\hline \multicolumn{2}{|c|}{ Age at alcohol initiation in years $(p=0.0009)$} & $\begin{array}{l}14 \\
(12- \\
14)\end{array}$ & $\begin{array}{l}16.5 \\
(15- \\
18)\end{array}$ & $\begin{array}{l}15.5(15- \\
18)\end{array}$ & $\begin{array}{l}17 \\
(16- \\
19)\end{array}$ & $\begin{array}{l}16.5 \\
(15- \\
18)\end{array}$ \\
\hline \multicolumn{2}{|l|}{ Drunk alcohol in the past 30 days } & $\begin{array}{l}5 \\
(45 \%)\end{array}$ & $\begin{array}{l}10 \\
(43 \%)\end{array}$ & $7(30 \%)$ & $\begin{array}{l}13 \\
(45 \%)\end{array}$ & $\begin{array}{l}35 \\
(41 \%)\end{array}$ \\
\hline \multicolumn{2}{|c|}{ Binge drinking in past 30 days $\left({ }^{2} p=0.076\right)$} & $\begin{array}{l}6 \\
(55 \%)\end{array}$ & $\begin{array}{l}6 \\
(26 \%)\end{array}$ & $4(17 \%)$ & $\begin{array}{l}5 \\
(17 \%)\end{array}$ & $\begin{array}{l}21 \\
(24 \%)\end{array}$ \\
\hline \multicolumn{7}{|l|}{ Illegal and Other Drug Use } \\
\hline \multicolumn{2}{|l|}{ Ever used cannabis (dagga) } & $\begin{array}{l}3 \\
(27 \%)\end{array}$ & $\begin{array}{l}7 \\
(30 \%)\end{array}$ & $5(22 \%)$ & $\begin{array}{l}8 \\
(28 \%)\end{array}$ & $\begin{array}{l}23 \\
(27 \%)\end{array}$ \\
\hline & $\begin{array}{l}\text { Age at first } \\
\text { cannabis } \\
\text { use }\end{array}$ & $\begin{array}{l}15 \\
(13- \\
16)\end{array}$ & $\begin{array}{l}16.5 \\
(14- \\
18)\end{array}$ & $\begin{array}{l}16(15- \\
16)\end{array}$ & $\begin{array}{l}18.5 \\
(16- \\
21)\end{array}$ & $\begin{array}{l}16 \\
(14.5- \\
18.5)\end{array}$ \\
\hline & $\begin{array}{l}\text { Cannabis } \\
\text { use in the } \\
\text { past year }\end{array}$ & $\begin{array}{l}3 \\
(27 \%)\end{array}$ & $\begin{array}{l}5 \\
(22 \%)\end{array}$ & $4(17 \%)$ & $\begin{array}{l}7 \\
(24 \%)\end{array}$ & $\begin{array}{l}19 \\
(22 \%)\end{array}$ \\
\hline
\end{tabular}

${ }^{1} P$-value associated with Kruskal-Wallis equality-of-populations rank test;

${ }^{2}$ Fisher's exact $p$-value;

${ }^{3}$ Inhalants = glue, aerosols, paint thinners, petrol or benzene;

${ }^{4}$ OTC $=$ over-the-counter. 


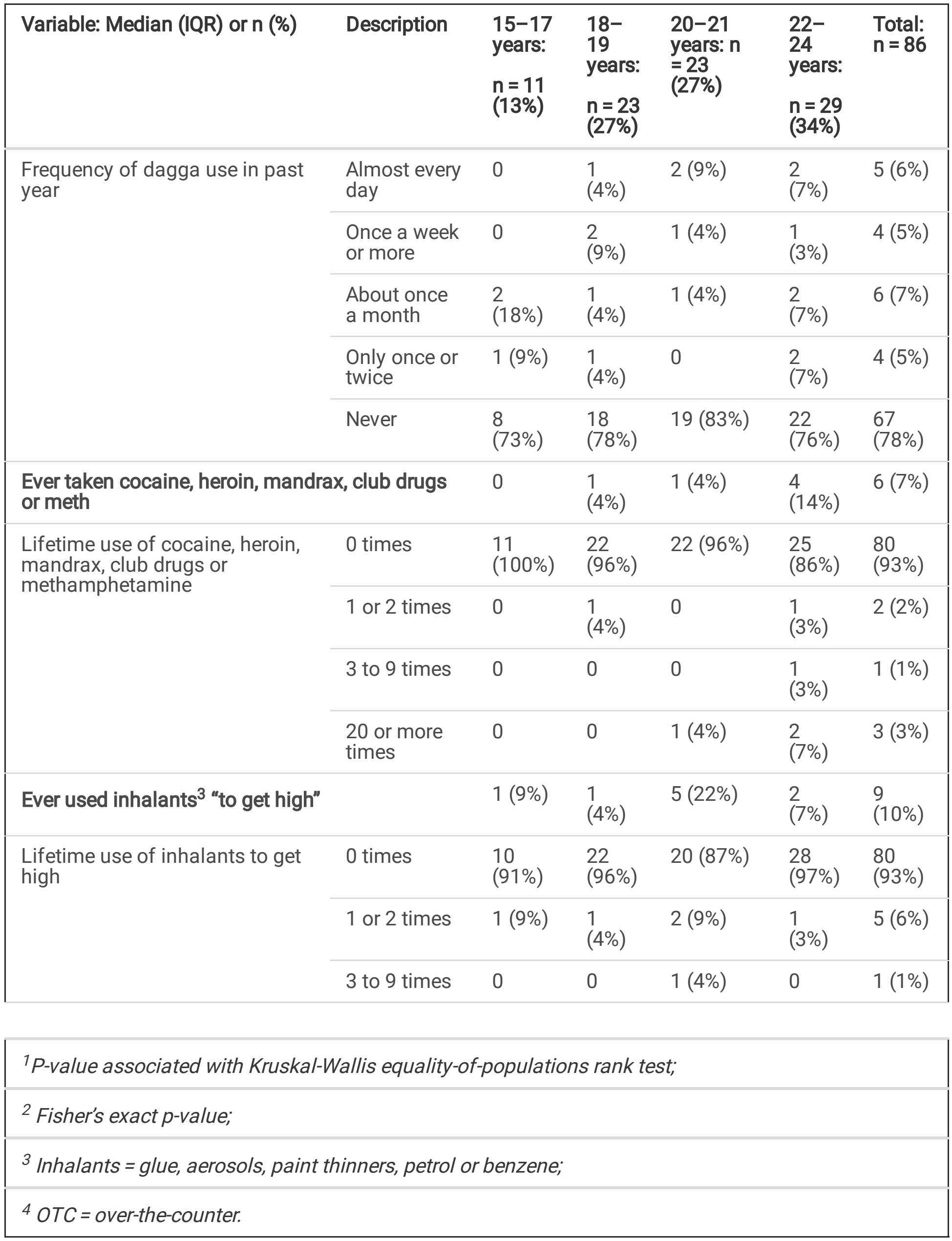




\begin{tabular}{|c|c|c|c|c|c|c|}
\hline Variable: Median (IQR) or $n(\%)$ & Description & $\begin{array}{l}15-17 \\
\text { years: } \\
\\
n=11 \\
(13 \%)\end{array}$ & $\begin{array}{l}18- \\
19 \\
\text { years: } \\
n=23 \\
(27 \%)\end{array}$ & $\begin{array}{l}20-21 \\
\text { years: } n \\
=23 \\
(27 \%)\end{array}$ & $\begin{array}{l}22- \\
24 \\
\text { years: } \\
n=29 \\
(34 \%)\end{array}$ & $\begin{array}{l}\text { Total: } \\
\mathrm{n}=\mathbf{8 6}\end{array}$ \\
\hline \multicolumn{2}{|c|}{$\begin{array}{l}\text { Ever used } \mathrm{OTC}^{4} \text { or prescription drugs "to get } \\
\text { high" }\end{array}$} & $\begin{array}{l}2 \\
(18 \%)\end{array}$ & $\begin{array}{l}4 \\
(17 \%)\end{array}$ & $5(22 \%)$ & 2 & $\begin{array}{l}13 \\
(15 \%)\end{array}$ \\
\hline \multirow[t]{3}{*}{ Lifetime use of OTC to get high } & 0 times & $\begin{array}{l}9 \\
(82 \%)\end{array}$ & $\begin{array}{l}20 \\
(87 \%)\end{array}$ & $20(87 \%)$ & $\begin{array}{l}27 \\
(93 \%)\end{array}$ & $\begin{array}{l}76 \\
(88 \%)\end{array}$ \\
\hline & 1 or 2 times & $\begin{array}{l}2 \\
(18 \%)\end{array}$ & $\begin{array}{l}3 \\
(13 \%)\end{array}$ & $2(9 \%)$ & 2 & $\begin{array}{l}9 \\
(10 \%)\end{array}$ \\
\hline & 3 to 9 times & 0 & 0 & $1(4 \%)$ & 0 & $1(1 \%)$ \\
\hline \multicolumn{2}{|c|}{$\begin{array}{l}\text { Ever used any illegal drugs or substance "to get } \\
\text { high" }\end{array}$} & $\begin{array}{l}4 \\
(36 \%)\end{array}$ & $\stackrel{9}{(39 \%)}$ & $8(35 \%)$ & $\begin{array}{l}10 \\
(34 \%)\end{array}$ & $\begin{array}{l}31 \\
(36 \%)\end{array}$ \\
\hline \multicolumn{7}{|c|}{${ }^{1} P$-value associated with Kruskal-Wallis equality-of-populations rank test; } \\
\hline \multicolumn{7}{|l|}{${ }^{2}$ Fisher's exact p-value; } \\
\hline \multicolumn{7}{|c|}{${ }^{3}$ Inhalants = glue, aerosols, paint thinners, petrol or benzene; } \\
\hline \multicolumn{7}{|l|}{${ }^{4}$ OTC $=$ over-the-counter. } \\
\hline
\end{tabular}

\section{Alcohol use}

Over two-thirds of participants reported ever drinking alcohol (68\%). Overall, the median age of first alcohol use was 16.5 years (IQR 15-18 years) with no significant difference by gender. However, age of first alcohol use was significantly earlier in the youngest age group with $82 \%$ of $15-17$-year olds reporting ever drinking alcohol and a median age of first alcohol use of 14 (IQR 12-14) years (See distribution in Table 5). Overall, $41 \%$ had drunk alcohol in the past 30 days and $24 \%$ reported binge drinking in the past month. Those who reported binge drinking were significantly younger (median 18.8 (IQR 16.9-21.5) years) than those who did not binge drink (median 21.1 (IQR 19.5-23.4) years) $(p=0.0152)$. Current drinking and binge drinking did not differ by gender. The youngest age group (15-17 years) reported the highest rates of risky drinking: $45 \%$ had drunk alcohol in the past 30 days (underage drinking) and $55 \%$ reported binge drinking compared to less than $30 \%$ in older age groups as shown in Table 5.

\section{Substance use}

More than a quarter (27\%) reported ever using cannabis. The median age at first cannabis use was 16 (IQR 14.5-18.5) years and did not differ significantly by gender. The frequency of cannabis use in the past year ranged from never (78\%) to almost every day (6\%) (See Table 4). Participants aged 18-19 and 20-21 years reported the most frequent use of cannabis with $13 \%$ reporting daily or weekly cannabis use each in the past year. Six participants (7\%), all over 18 years old, reported ever taking "hard" drugs 
(cocaine, methamphetamine or mandrax); three of whom reported taking hard drugs 20 or more times in their lifetime. Nine participants (10\%) reported ever using inhalants such as glue, aerosols, paint thinners, petrol or benzene "to get high" and 15\% reported ever using over-the-counter or prescription drugs at least once or twice "to get high". Overall, more males (53\%) reported ever using any illegal drugs or substances "to get high" compared to females (31\%).

\section{Comorbidities and symptom screening Pre-existing diagnosis}

Overall, $35 \%$ reported a previous comorbidity diagnosis (95\% Cl: $25-45 \%)$. The most common preexisting diagnoses or previous comorbidities were TB (23\%; $95 \% \mathrm{Cl}$ : $15-33 \%)$ and depression/anxiety (8.7\%; $95 \% \mathrm{Cl} 3.8-16 \%)$, followed by asthma (4.3\%; $95 \% \mathrm{Cl} 1.2-11 \%)$ and high blood pressure $(3.2 \%$; $95 \%$ $\mathrm{Cl}: 0.68-9.2 \%)$. There were no significant differences in the proportions of male and female participants diagnosed with pre-existing conditions (Table 6). 
Table 6

Comorbidities, Pre-existing diagnoses, Measured clinical signs and symptoms by Gender

\section{Variable: median (IQR) or $\mathrm{n}(\%)$ or proportion [95\% Cl]}

Self-reported preexisting diagnosis: prop $[95 \% \mathrm{Cl}]^{2}$
Male: $\mathrm{n}=\mathbf{2 2}$

$0.45[0.24-$

$0.68]$

$0.32[0.14-$

$0.55]$

Tuberculosis

Depression or Anxiety

$0.14[0.029-$ $0.35]$

Asthma

0.045

$[0.0012-$

$0.23]$

High Blood Pressure

Diabetes
0 [0-0.15]

$0[0-0.15]$
Female: $\mathrm{n}=$ 70

$0.31[0.21-$

$0.44]$

$0.2[0.11-$

$0.31]$

0.071

[0.024-0.16]

0.043

[0.0089-

0.12]

\subsection{3}

[0.089-0.12]
Total $\mathbf{n}=92$

pvalue $^{1}$

0.228

$0.35[0.25-$

$0.45]$

0.229

$0.23[0.15-$

0.33 ]

0.332

0.087

[0.038-0.16]

0.946

0.043

[0.012-0.11]

0.327

0.033
$[0.0068-$
$0.092]$

$\begin{array}{ll}0.014 & 0.011 \\ {[0.00036-} & {[0.00028-} \\ 0.077] & 0.061]\end{array}$

0.576

Respiratory Symptoms over past 3

months $(n=89)$ : prop [95\% Cl]

$0.10[0.012-$

$0.30]$

$0.13[0.062-$

$0.24]$

$0.12[0.063-\quad 0.651$

$0.21]$

Diabetes Symptoms over past 3 months: prop [95\% Cl]

$0.29[0.11-$

$0.52]$

$0.24[0.14-$

$0.35]$

$0.25[0.16-$

$0.35]$

Symptoms Frequent urination

$1(5 \%)$

$3(4 \%)$

$4(4 \%)$

0.969

n (\%)

Increased thirst

$2(9 \%)$

$7(10 \%)$

$9(10 \%)$

0.900

Weight loss $>1.5 \mathrm{~kg}$ in past month

0

$6(9 \%)$

$6(7 \%)$

0.152

Unexplained fatigue

$5(23 \%)$

$9(13 \%)$

$13(15 \%)$

0.261

Blurry vision

$2(10 \%)$

$5(7 \%)$

$7(8 \%)$

0.747

Family history of diabetes: prop [95\% Cl]

$0.38[0.18-$

$0.24[0.14-$ $0.35]$

$0.27[0.18-$

0.189

Wilcoxon rank-sum test $p$-value for continuous variables and Pearson X2 test for categorical variables;

2 Proportion and Binomial exact 95\% Confidence interval;

${ }^{3}$ Blood glucose measured only if had one or more symptoms of diabetes and OR family history;

${ }^{4}$ Abdominal obesity: $W C>88 \mathrm{~cm}$ in females, $W C>102 \mathrm{~cm}$ in males. 


\begin{tabular}{|c|c|c|c|c|c|}
\hline \multicolumn{2}{|c|}{$\begin{array}{l}\text { Variable: median (IQR) or } \mathrm{n}(\%) \text { or } \\
\text { proportion [ } 95 \% \mathrm{Cl}]\end{array}$} & Male: $n=22$ & $\begin{array}{l}\text { Female: } \mathrm{n}= \\
70\end{array}$ & Total $\mathrm{n}=92$ & $\begin{array}{l}\text { p- } \\
\text { value }^{1}\end{array}$ \\
\hline \multicolumn{2}{|c|}{$\begin{array}{l}\text { Random Blood Glucose in } \mathrm{mmol} / \mathrm{l}(\mathrm{n}= \\
31)^{3} \text { median (IQR) }\end{array}$} & $\begin{array}{l}3.7(0.75- \\
4.95)\end{array}$ & $4.8(4.0-5.5)$ & $4.7(3.1-5.3)$ & 0.1672 \\
\hline \multicolumn{2}{|c|}{$\begin{array}{l}\text { CESD-10 Depression Score }(n=82) \\
\text { median (IQR) }\end{array}$} & $8(4-9)$ & $10(6-14)$ & $9(6-14)$ & 0.055 \\
\hline & $\begin{array}{l}\text { Significant depression } \\
\text { (CESD } \geq 10) \text { : prop }[95 \% \text { CI] }\end{array}$ & $\begin{array}{l}0.19[0.054- \\
0.42]\end{array}$ & $\begin{array}{l}0.51[0.38- \\
0.64]\end{array}$ & $\begin{array}{l}0.43[0.32- \\
0.54]\end{array}$ & 0.011 \\
\hline \multicolumn{2}{|c|}{$\begin{array}{l}\text { Kessler Psychological Distress Score } \\
(\mathrm{K} 10)(\mathrm{n}=85) \text { median (IQR) }\end{array}$} & $17(13-23)$ & $19(13-25)$ & $19(13-25)$ & 0.959 \\
\hline \multirow{4}{*}{$\begin{array}{l}\text { Categories } \\
\mathrm{n}(\%)\end{array}$} & Mentally well $($ K10 < 20) & $12(57 \%)$ & $35(55 \%)$ & 47 (55\%) & \multirow[t]{4}{*}{0.407} \\
\hline & Mild distress (K10 20-24) & $4(19 \%)$ & $10(16 \%)$ & $14(16 \%)$ & \\
\hline & $\begin{array}{l}\text { Moderate distress (K10 } \\
25-29)\end{array}$ & $2(10 \%)$ & $15(23 \%)$ & $17(20 \%)$ & \\
\hline & Severe distress $(K 10 \geq 30)$ & $3(14 \%)$ & $4(6 \%)$ & $7(8 \%)$ & \\
\hline \multicolumn{6}{|c|}{ Measured Clinical Signs } \\
\hline \multicolumn{2}{|c|}{$\mathrm{BMI}$ in $\mathrm{kg} / \mathrm{m}^{2}$; median (IQR) } & $\begin{array}{l}21.0(19.1- \\
22.6)\end{array}$ & $\begin{array}{l}23.3(20.2- \\
27.2)\end{array}$ & $\begin{array}{l}22.8(19.9- \\
26.2)\end{array}$ & 0.0102 \\
\hline \multirow{4}{*}{$\begin{array}{l}\text { Categories } \\
\mathrm{n}(\%)\end{array}$} & Underweight $(\mathrm{BMI}<18.5)$ & $5(23 \%)$ & $5(7 \%)$ & $10(11 \%)$ & \multirow[t]{4}{*}{0.032} \\
\hline & $\begin{array}{l}\text { Normal weight }(18.5 \leq \\
\mathrm{BMI}<25)\end{array}$ & $14(64 \%)$ & $33(49 \%)$ & $47(53 \%)$ & \\
\hline & $\begin{array}{l}\text { Overweight }(25 \leq \mathrm{BMI}< \\
30)\end{array}$ & $3(14 \%)$ & $19(28 \%)$ & $22(25 \%)$ & \\
\hline & Obese $(\mathrm{BMI} \geq 30)$ & 0 & $10(15 \%)$ & $10(11 \%)$ & \\
\hline \multicolumn{2}{|c|}{$\begin{array}{l}\text { Waist circumference (WC) in cm; median } \\
\text { (IQR) }\end{array}$} & $\begin{array}{l}74(71.5- \\
81.0)\end{array}$ & $\begin{array}{l}78.4(71.5- \\
88.0)\end{array}$ & $\begin{array}{l}76(71.5- \\
87)\end{array}$ & 0.2135 \\
\hline & Abdominal obesity ${ }^{4}$ & $1(5 \%)$ & $17(24 \%)$ & $18(20 \%)$ & 0.049 \\
\hline \multicolumn{2}{|c|}{ Hip circumference in cm; median (IQR) } & $87.5(84-94)$ & $\begin{array}{l}97.5(90.5- \\
108)\end{array}$ & $\begin{array}{l}95(86.75- \\
106.5)\end{array}$ & 0.0011 \\
\hline
\end{tabular}

Wilcoxon rank-sum test $p$-value for continuous variables and Pearson X2 test for categorical variables;

2 Proportion and Binomial exact 95\% Confidence interval;

${ }^{3}$ Blood glucose measured only if had one or more symptoms of diabetes and OR family history;

${ }^{4}$ Abdominal obesity: $W C>88 \mathrm{~cm}$ in females, $W C>102 \mathrm{~cm}$ in males. 


\begin{tabular}{|c|c|c|c|c|c|}
\hline \multicolumn{2}{|c|}{$\begin{array}{l}\text { Variable: median (IQR) or } \mathrm{n}(\%) \text { or } \\
\text { proportion }[95 \% \mathrm{Cl}]\end{array}$} & Male: $n=22$ & $\begin{array}{l}\text { Female: } n= \\
70\end{array}$ & Total $\mathbf{n}=92$ & $\begin{array}{l}\text { p- } \\
\text { value }^{1}\end{array}$ \\
\hline \multicolumn{2}{|c|}{ Waist-hip ratio (WHR) median (IQR) } & $\begin{array}{l}0.85(0.82- \\
0.87)\end{array}$ & $\begin{array}{l}0.82(0.77- \\
0.85)\end{array}$ & $\begin{array}{l}0.82(0.78- \\
0.87)\end{array}$ & 0.0265 \\
\hline \multicolumn{2}{|c|}{$\begin{array}{l}\text { Waist-to-height ratio (WHtR) median } \\
\text { (IQR) }\end{array}$} & $\begin{array}{l}0.43(0.42- \\
0.48)\end{array}$ & $\begin{array}{l}0.49(0.45- \\
0.56)\end{array}$ & $\begin{array}{l}0.48(0.44- \\
0.54)\end{array}$ & 0.0006 \\
\hline \multirow[t]{2}{*}{$\begin{array}{l}\text { Central } \\
\text { obesity }\end{array}$} & $\begin{array}{l}\text { WHR }>0.85 \text { in females } \\
\text { and }>0.95 \text { in males }\end{array}$ & 0 & $19(27 \%)$ & $19(21 \%)$ & 0.007 \\
\hline & WHtR $>0.5$ & $3(14 \%)$ & $31(44 \%)$ & $34(37 \%)$ & 0.020 \\
\hline \multirow{2}{*}{\multicolumn{2}{|c|}{$\begin{array}{l}\text { Blood Pressure in mmHg } \\
\text { (Systolic/ Diastolic Blood Pressure) } \\
\text { median (IQR) }\end{array}$}} & $119.5 / 74.75$ & $115 / 74$ & $117.5 / 74.25$ & 0.9635 \\
\hline & & $\begin{array}{l}(110 / 66.5- \\
131.5 / 80.5)\end{array}$ & $\begin{array}{l}(109.5 / 68.5- \\
124.5 / 78)\end{array}$ & $\begin{array}{l}(109.5 / 68- \\
125.5 / 79)\end{array}$ & 0.2097 \\
\hline \multirow{3}{*}{$\begin{array}{l}\text { Categories } \\
\mathrm{n}(\%)\end{array}$} & $\begin{array}{l}\text { Normal BP: SBP }<130 \& \\
\text { DBP }<85\end{array}$ & $13(59 \%)$ & $56(80 \%)$ & $69(75 \%)$ & \multirow[t]{3}{*}{0.056} \\
\hline & $\begin{array}{l}\text { Elevated BP: SBP } 130- \\
139 \text { or DBP } 85-89\end{array}$ & $6(27 \%)$ & $12(17 \%)$ & $18(20 \%)$ & \\
\hline & $\begin{array}{l}\text { Hypertension: SBP } 140- \\
\text { 159/ DBP } 90-99\end{array}$ & $3(14 \%)$ & $2(3 \%)$ & $5(5 \%)$ & \\
\hline \multicolumn{6}{|c|}{$\begin{array}{l}\text { Wilcoxon rank-sum test p-value for continuous variables and Pearson } X 2 \text { test for categorical } \\
\text { variables; }\end{array}$} \\
\hline \multicolumn{6}{|c|}{2 Proportion and Binomial exact 95\% Confidence interval; } \\
\hline \multicolumn{6}{|c|}{${ }^{3}$ Blood glucose measured only if had one or more symptoms of diabetes and OR family history; } \\
\hline \multicolumn{6}{|c|}{${ }^{4}$ Abdominal obesity: $W C>88 \mathrm{~cm}$ in females, $W C>102 \mathrm{~cm}$ in males. } \\
\hline
\end{tabular}

\section{Respiratory symptoms}

Eleven participants (12\%; $95 \% \mathrm{Cl}: 6.3-21 \%)$ reported experiencing one or more of the following respiratory symptoms over the past three months: shortness of breath (3\%), chest tightness (3\%), prolonged cough with sputum for more than two weeks $(7 \%)$, or difficulty breathing (3\%). Of those who reported difficulty breathing, 2/3 reported it to be worse at night, with wheezing or whistling in the chest or occurring during exercise. Peak flow measurement was done for five participants who reported respiratory symptoms but had no known asthma diagnosis. All the measurements were within the normal - green- peak flow zone.

\section{Diabetes}

Only one participant (who also reported diabetes symptoms) reported a previous diabetes diagnosis. A quarter of participants ( $95 \% \mathrm{Cl}: 16-35 \%$ ) reported experiencing one or more of the following diabetesrelated symptoms over the past three months: frequent urination (4\%), increased thirst (10\%), unexplained weight loss of more than $1.5 \mathrm{~kg}$ in the last month (7\%), unexplained fatigue (15\%) and blurry vision (8\%). 
Younger age groups (15-17 years and 18-19 years) reported more diabetes-related symptoms compared to older age groups (See Table 7). Overall, $27 \%$ reported a family history of diabetes ( $95 \% \mathrm{Cl}$ : $18-37 \%)$. Of those with reported symptoms or a reported family history of diabetes who had their blood glucose measured $(n=31)$, none had a measured random blood glucose of more than $7 \mathrm{mmol} / \mathrm{L}$. 
Table 7

Comorbidities, Pre-existing diagnoses, Measured clinical signs and symptoms by Age Group

\begin{tabular}{|c|c|c|c|c|c|c|}
\hline \multirow{2}{*}{\multicolumn{2}{|c|}{ Variable: median (IQR) or $\mathrm{n}(\%)$}} & $\begin{array}{l}15-17 \\
\text { years: }\end{array}$ & $\begin{array}{l}18-19 \\
\text { years: }\end{array}$ & $\begin{array}{l}20-21 \\
\text { years: }\end{array}$ & $\begin{array}{l}22-24 \\
\text { years: }\end{array}$ & $\begin{array}{l}\text { Total: } \mathrm{n} \\
=92\end{array}$ \\
\hline & & $\begin{array}{l}n=14 \\
(15 \%)\end{array}$ & $\begin{array}{l}n=23 \\
(25 \%)\end{array}$ & $\begin{array}{l}n=26 \\
(28 \%)\end{array}$ & $\begin{array}{l}n=29 \\
(32 \%)\end{array}$ & \\
\hline \multicolumn{2}{|c|}{$\begin{array}{l}\text { Self-reported past } \\
\text { diagnosis: } \mathbf{n}(\%)\end{array}$} & & $9(39 \%)$ & $9(35 \%)$ & $13(45 \%)$ & $32(35 \%)$ \\
\hline \multicolumn{2}{|r|}{ Tuberculosis } & $1(7 \%)$ & $5(22 \%)$ & $6(23 \%)$ & $9(31 \%)$ & $21(23 \%)$ \\
\hline \multicolumn{2}{|r|}{ Depression or Anxiety } & 0 & $4(17 \%)$ & $2(8 \%)$ & $2(7 \%)$ & $8(9 \%)$ \\
\hline \multicolumn{2}{|r|}{ Asthma } & 0 & $2(9 \%)$ & $2(8 \%)$ & 0 & $4(4 \%)$ \\
\hline \multicolumn{2}{|r|}{ High Blood Pressure } & 0 & 0 & $1(4 \%)$ & $2(7 \%)$ & $3(3 \%)$ \\
\hline \multicolumn{2}{|r|}{ Diabetes } & 0 & $1(11 \%)$ & 0 & 0 & $1(3 \%)$ \\
\hline \multicolumn{2}{|c|}{$\begin{array}{l}\text { Respiratory Symptoms over the past } 3 \\
\text { months }(n=89)\end{array}$} & $4(29 \%)$ & $4(17 \%)$ & $2(8 \%)$ & $1(3 \%)$ & $11(12 \%)$ \\
\hline \multicolumn{2}{|c|}{$\begin{array}{l}\text { Diabetes Symptoms over past } 3 \\
\text { months }(p=0.047)\end{array}$} & $6(46 \%)$ & $8(35 \%)$ & $4(16 \%)$ & $4(14 \%)$ & $23(25 \%)$ \\
\hline \multirow{5}{*}{$\begin{array}{l}\text { Symptoms } \\
\mathrm{n}(\%)\end{array}$} & $\begin{array}{l}\text { Frequent urination }(p= \\
0.026)\end{array}$ & $2(15 \%)$ & $2(9 \%)$ & 0 & 0 & $4(4 \%)$ \\
\hline & $\begin{array}{l}\text { Increased thirst }(p= \\
0.011)\end{array}$ & $4(31 \%)$ & $3(13 \%)$ & $2(8 \%)$ & 0 & $9(10 \%)$ \\
\hline & $\begin{array}{l}\text { Weight loss }>1.5 \mathrm{~kg} \text { in } \\
\text { past month }\end{array}$ & $1(8 \%)$ & $2(9 \%)$ & $1(4 \%)$ & $2(7 \%)$ & $6(7 \%)$ \\
\hline & Unexplained fatigue & $4(31 \%)$ & $5(22 \%)$ & $4(16 \%)$ & $1(3 \%)$ & $14(16 \%)$ \\
\hline & Blurry vision & $1(8 \%)$ & $3(14 \%)$ & $1(4 \%)$ & $2(7 \%)$ & $7(8 \%)$ \\
\hline \multicolumn{2}{|c|}{ Family history of diabetes } & $6(46 \%)$ & $7(30 \%)$ & $4(16 \%)$ & $7(24 \%)$ & $24(27 \%)$ \\
\hline \multicolumn{2}{|c|}{$\begin{array}{l}\text { Random Blood Glucose in } \mathrm{mmol} / \mathrm{I}(\mathrm{n}= \\
31)^{3} \text { median (IQR) }\end{array}$} & $\begin{array}{l}3.65(1- \\
4.9)\end{array}$ & $\begin{array}{l}4.4 \\
(2.75- \\
5.5)\end{array}$ & $\begin{array}{l}5(2.4- \\
5.45)\end{array}$ & $\begin{array}{l}5.2(4.7- \\
5.5)\end{array}$ & $\begin{array}{l}4.7(3.1- \\
5.3)\end{array}$ \\
\hline \multicolumn{2}{|c|}{$\begin{array}{l}\text { CESD-10 Depression Score }(\mathrm{n}=81) \\
\text { median (IQR) }\end{array}$} & $9(6-12)$ & $\begin{array}{l}10.5 \\
(7.5-14)\end{array}$ & $\begin{array}{l}9(7.5- \\
13.5)\end{array}$ & $\begin{array}{l}8.5(6- \\
12)\end{array}$ & $9(6-14)$ \\
\hline
\end{tabular}

Binomial exact 95\% Confidence interval;

2 P-value derived from Fisher's exact text;

${ }^{3}$ Blood glucose measured only if had one or more symptoms of diabetes and OR family history;

${ }^{4}$ Abdominal obesity: $W C>88 \mathrm{~cm}$ female, $W C>102 \mathrm{~cm}$ in male. 


\begin{tabular}{|c|c|c|c|c|c|c|}
\hline \multicolumn{2}{|c|}{ Variable: median (IQR) or $\mathrm{n}(\%)$} & \multirow{2}{*}{$\begin{array}{l}15-17 \\
\text { years: } \\
n=14 \\
(15 \%)\end{array}$} & \multirow{2}{*}{$\begin{array}{l}18-19 \\
\text { years: } \\
n=23 \\
(25 \%)\end{array}$} & \multirow{2}{*}{$\begin{array}{l}20-21 \\
\text { years: } \\
n=26 \\
(28 \%)\end{array}$} & \multirow{2}{*}{$\begin{array}{l}22-24 \\
\text { years: } \\
n=29 \\
(32 \%)\end{array}$} & \multirow[t]{2}{*}{$\begin{array}{l}\text { Total: } \mathbf{n} \\
=92\end{array}$} \\
\hline & & & & & & \\
\hline & $\begin{array}{l}\text { Significant depression } \\
(C E S D \geq 10)\end{array}$ & $5(38 \%)$ & $12(60 \%)$ & $8(33 \%)$ & $10(42 \%)$ & $35(43 \%)$ \\
\hline $\begin{array}{l}\text { Kessler Dist } \\
\text { median (IQF }\end{array}$ & ss Score $(K 10)(n=85)$ & $\begin{array}{l}15(14- \\
24)\end{array}$ & $\begin{array}{l}19(15- \\
25)\end{array}$ & $\begin{array}{l}18(12- \\
25)\end{array}$ & $\begin{array}{l}18.5 \\
(13-25)\end{array}$ & $\begin{array}{l}19(13- \\
25)\end{array}$ \\
\hline \multirow{4}{*}{$\begin{array}{l}\text { Categories } \\
\mathrm{n}(\%)\end{array}$} & Mentally well $(\mathrm{K} 10<20)$ & $7(54 \%)$ & $12(55 \%)$ & $13(54 \%)$ & $15(58 \%)$ & $47(55 \%)$ \\
\hline & $\begin{array}{l}\text { Mild distress (K10 20- } \\
\text { 24) }\end{array}$ & $3(23 \%)$ & $3(14 \%)$ & $5(21 \%)$ & $3(12 \%)$ & $14(16 \%)$ \\
\hline & $\begin{array}{l}\text { Moderate distress (K10 } \\
25-29)\end{array}$ & $1(8 \%)$ & $5(23 \%)$ & $4(17 \%)$ & $7(27 \%)$ & $17(20 \%)$ \\
\hline & $\begin{array}{l}\text { Severe distress }(\mathrm{K} 10 \geq \\
30)\end{array}$ & $2(15 \%)$ & $2(9 \%)$ & $2(8 \%)$ & $1(4 \%)$ & $7(8 \%)$ \\
\hline \multicolumn{7}{|c|}{ Measured Clinical Signs } \\
\hline \multicolumn{2}{|c|}{ BMI in $\mathrm{kg} / \mathrm{m}^{2}(\mathrm{n}=87)$; median (IQR) } & $\begin{array}{l}20.8 \\
(19.5- \\
24.1)\end{array}$ & $\begin{array}{l}23.2 \\
(19.3- \\
26.4)\end{array}$ & $\begin{array}{l}22.1 \\
(20.6- \\
25.9)\end{array}$ & $\begin{array}{l}24.2 \\
(20.2- \\
27.1)\end{array}$ & $\begin{array}{l}22.8 \\
(19.9- \\
26.2)\end{array}$ \\
\hline \multirow{4}{*}{$\begin{array}{l}\text { Categories } \\
\mathrm{n}(\%)\end{array}$} & $\begin{array}{l}\text { Underweight }(\mathrm{BMI}< \\
\text { 18.5) }\end{array}$ & $2(14 \%)$ & $4(19 \%)$ & $1(4 \%)$ & $3(10 \%)$ & $10(11 \%)$ \\
\hline & $\begin{array}{l}\text { Normal weight }(18.5 \leq \\
\mathrm{BMI}<25)\end{array}$ & $9(64 \%)$ & $8(38 \%)$ & $15(60 \%)$ & $15(52 \%)$ & $47(53 \%)$ \\
\hline & $\begin{array}{l}\text { Overweight }(25 \leq \mathrm{BMI}< \\
30)\end{array}$ & $1(7 \%)$ & $7(33 \%)$ & $6(24 \%)$ & $8(28 \%)$ & $22(25 \%)$ \\
\hline & Obese $(\mathrm{BMI} \geq 30)$ & $2(14 \%)$ & $2(10 \%)$ & $3(12 \%)$ & $3(10 \%)$ & $10(11 \%)$ \\
\hline \multicolumn{2}{|c|}{$\begin{array}{l}\text { Waist circumference (WC) in cm; } \\
\text { median (IQR) }\end{array}$} & $\begin{array}{l}74.5 \\
(71-76)\end{array}$ & $\begin{array}{l}74.6 \\
(67.5- \\
88)\end{array}$ & $\begin{array}{l}78.8 \\
(73-85)\end{array}$ & $\begin{array}{l}79(71- \\
88)\end{array}$ & $\begin{array}{l}76 \\
(71.5- \\
87)\end{array}$ \\
\hline & Abdominal obesity ${ }^{4}$ & $2(15 \%)$ & $5(23 \%)$ & $4(12 \%)$ & $7(24 \%)$ & $17(19 \%)$ \\
\hline
\end{tabular}

Binomial exact 95\% Confidence interval;

2 P-value derived from Fisher's exact text;

${ }^{3}$ Blood glucose measured only if had one or more symptoms of diabetes and OR family history;

${ }^{4}$ Abdominal obesity: $W C>88 \mathrm{~cm}$ female, $W C>102 \mathrm{~cm}$ in male. 


\begin{tabular}{|c|c|c|c|c|c|c|}
\hline \multirow{2}{*}{\multicolumn{2}{|c|}{ Variable: median (IQR) or $\mathrm{n}(\%)$}} & $\begin{array}{l}\text { 15-17 } \\
\text { years: }\end{array}$ & $\begin{array}{l}\text { 18-19 } \\
\text { years: }\end{array}$ & $\begin{array}{l}20-21 \\
\text { years: }\end{array}$ & $\begin{array}{l}22-24 \\
\text { years: }\end{array}$ & $\begin{array}{l}\text { Total: } \mathbf{n} \\
=92\end{array}$ \\
\hline & & $\begin{array}{l}n=14 \\
(15 \%)\end{array}$ & $\begin{array}{l}n=23 \\
(25 \%)\end{array}$ & $\begin{array}{l}n=26 \\
(28 \%)\end{array}$ & $\begin{array}{l}n=29 \\
(32 \%)\end{array}$ & \\
\hline \multicolumn{2}{|c|}{ Hip circumference in cm; median (IQR) } & $\begin{array}{l}91 \\
(83.5- \\
104)\end{array}$ & $\begin{array}{l}90.5 \\
(84.8- \\
107)\end{array}$ & $\begin{array}{l}96.7 \\
(93- \\
107)\end{array}$ & $\begin{array}{l}95(90- \\
105)\end{array}$ & $\begin{array}{l}95 \\
(86.5- \\
106)\end{array}$ \\
\hline \multicolumn{2}{|c|}{ Waist-hip ratio (WHR); median (IQR) } & $\begin{array}{l}0.83 \\
(0.79- \\
0.87)\end{array}$ & $\begin{array}{l}0.83 \\
(0.77- \\
0.87)\end{array}$ & $\begin{array}{l}0.82 \\
(0.78- \\
0.85)\end{array}$ & $\begin{array}{l}0.82 \\
(0.79- \\
0.87)\end{array}$ & $\begin{array}{l}0.82 \\
(0.78- \\
0.87)\end{array}$ \\
\hline \multicolumn{2}{|c|}{$\begin{array}{l}\text { Waist-to-height ratio (WHtR); median } \\
\text { (IQR) }\end{array}$} & $\begin{array}{l}0.47 \\
(0.43- \\
0.48)\end{array}$ & $\begin{array}{l}0.45 \\
(0.43- \\
0.53)\end{array}$ & $\begin{array}{l}0.48 \\
(0.44- \\
0.52)\end{array}$ & $\begin{array}{l}0.49 \\
(0.44- \\
0.57)\end{array}$ & $\begin{array}{l}0.48 \\
(0.44- \\
0.54)\end{array}$ \\
\hline \multirow[t]{2}{*}{$\begin{array}{l}\text { Central } \\
\text { obesity }\end{array}$} & $\begin{array}{l}\text { WHR }>0.85 \text { in female } \\
\text { and }>0.95 \text { in male }\end{array}$ & $3(23 \%)$ & $5(23 \%)$ & $3(8 \%)$ & $8(28 \%)$ & $18(20 \%)$ \\
\hline & WHtR $>0.5$ & $2(14 \%)$ & $9(39 \%)$ & $11(42 \%)$ & $12(41 \%)$ & $34(37 \%)$ \\
\hline \multirow{2}{*}{\multicolumn{2}{|c|}{$\begin{array}{l}\text { Systolic Blood Pressure in mmHg; } \\
\text { median (IQR) }\end{array}$}} & 117.5 & 118 & 118 & 117 & 117.5 \\
\hline & & $\begin{array}{l}(112- \\
125.5)\end{array}$ & $\begin{array}{l}(108.5- \\
124.5)\end{array}$ & $\begin{array}{l}(111- \\
131)\end{array}$ & $\begin{array}{l}(107.5- \\
123.5)\end{array}$ & $\begin{array}{l}(109.5- \\
125.5)\end{array}$ \\
\hline \multicolumn{2}{|c|}{$\begin{array}{l}\text { Diastolic Blood Pressure in mmHg; } \\
\text { median (IQR) }\end{array}$} & $\begin{array}{l}73.5 \\
(66.5- \\
77)\end{array}$ & $\begin{array}{l}75(67- \\
76.5)\end{array}$ & $\begin{array}{l}76(71- \\
80)\end{array}$ & $\begin{array}{l}74 \\
(68.5- \\
80)\end{array}$ & $\begin{array}{l}74.25 \\
(68- \\
79.5)\end{array}$ \\
\hline \multirow{3}{*}{$\begin{array}{l}\text { Categories } \\
\mathrm{n}(\%)\end{array}$} & $\begin{array}{l}\text { Normal BP: } \mathrm{SBP}<130 \& \\
\mathrm{DBP}<85\end{array}$ & $11(79 \%)$ & $18(78 \%)$ & $18(69 \%)$ & $22(76 \%)$ & $69(75 \%)$ \\
\hline & $\begin{array}{l}\text { Elevated BP: SBP } 130- \\
139 \text { or DBP } 85-89\end{array}$ & $3(21 \%)$ & $4(17 \%)$ & $6(23 \%)$ & $5(17 \%)$ & $18(20 \%)$ \\
\hline & $\begin{array}{l}\text { Hypertension: SBP } \\
\text { 140-159/ DBP } 90-99\end{array}$ & 0 & $1(4 \%)$ & $2(8 \%)$ & $2(7 \%)$ & $5(5 \%)$ \\
\hline \multicolumn{7}{|c|}{ Binomial exact 95\% Confidence interval; } \\
\hline \multicolumn{7}{|c|}{2 P-value derived from Fisher's exact text; } \\
\hline \multicolumn{7}{|c|}{${ }^{3}$ Blood glucose measured only if had one or more symptoms of diabetes and OR family history; } \\
\hline \multicolumn{7}{|c|}{${ }^{4}$ Abdominal obesity: $W C>88 \mathrm{~cm}$ female, $W C>102 \mathrm{~cm}$ in male. } \\
\hline
\end{tabular}

\section{Mental health screening}

According to the CESD-10 depression scale, $43 \%$ of participants were classified as experiencing significant depression in the past week (95\% Cl: 32-54\%). CESD-10 scores differed significantly by gender with female participants more likely to report depressive symptoms compared to males as shown in Table 6 and Fig. 4 (51\% versus 19\%, $p=0.011$ ). Of those with significant depression, $6 / 35(17 \%)$ 
reported a previous diagnosis of depression or anxiety. There were no significant differences in depression scores by age. Almost half the participants reported some level of psychological distress over the past month (45\%; $95 \% \mathrm{Cl}: 34-56 \%)$ with $8 \%$ indicating symptoms of severe psychological distress on the K10 scale (See Fig. 5). Only $11 \%$ of those with mild, moderate or severe psychological distress reported previously being diagnosed with depression or anxiety. The prevalence of psychological distress symptoms was comparable across age groups (See Table 7).

\section{Measured Clinical Signs}

\section{Overweight/obesity}

Overall, $36 \%$ of participants were either overweight $(25 \%)$ or obese $(11 \%)$, with significant differences by gender; $14 \%$ males and $43 \%$ of females (See Fig. 7). The median BMI for males (21.0; IQR 19.1$22.6 \mathrm{~kg} / \mathrm{m}^{2}$ ) was significantly lower than for females (23.3; IQR $20.2-27.2 \mathrm{~kg} / \mathrm{m}^{2}$ ). A greater proportion of participants aged 18-19 years were overweight or obese (43\%) compared to the other age groups (23\% in 15-17-year olds; $33 \%$ in 20-21-year olds and 38\% in 22-24-year olds) (See Table 7).

\section{Abdominal obesity}

There was a markedly significant difference in the waist-hip ratio (WHR) of males and females. A total of $27 \%$ of females and no males had central obesity as shown in Table 6 . Using the WHtR, $44 \%$ of females had central obesity compared to $14 \%$ of males. Waist circumference increased with age, with those aged 22-24 years having the highest waist circumference of $79 \mathrm{~cm}$ (IQR 71-88), but WHR did not differ by age. It is important to note that $12(26 \%)$ participants with normal BMI had abnormal WHR and/or WHtR and could be classified as having central obesity.

\section{Measured Blood Pressure}

Overall, $75 \%$ of participants had a normal blood pressure, $20 \%$ had an elevated blood pressure and $5 \%$ had hypertension. Figure 6 shows that the prevalence of elevated blood pressure and hypertension was higher in males compared to females ( $41 \%$ and $20 \%$ respectively, $p=0.0367)$. Systolic and diastolic blood pressure showed an increasing trend with age (Table 7). The median age of those with elevated blood pressure or hypertension was 21.2 (IQR 18.8-22.7) years, similar to those with normal blood pressure (median 20.2, IQR: 19.0-22.8 years). Of those with elevated blood pressure or hypertension, 11 (35\%) were overweight or obese and $2(6 \%)$ reported a previous diagnosis of hypertension (data not shown in tables).

\section{Discussion}

This study describes the prevalence of NCDs and NCD risk factors among South African AYLHIV in an urban setting. Previous studies in sub-Saharan Africa have described NCD comorbidities in adults living with HIV [44, 109-112]. Besides mental health [113-121] and lung diseases [122-126], few studies have assessed the prevalence of NCDs and particularly NCD risk factors in AYLHIV in SSA other than as incidental findings [127-134]. Risk behaviour research on AYLHIV in sub-Saharan Africa has 
predominantly focused on sexual risk behaviour [135]. We therefore set out to investigate NCD risk factors given the emerging NCD epidemic in SSA occurring against a background of a high HIV burden and increased comorbidity risk in PLHIV.

We highlight several key findings. Almost half of our participants faced multiple deprivations of poverty, significant symptoms of depression and psychological distress, and multiple risk factors for NCDs. More than a third were overweight or obese, a third had insufficient levels of weekly physical activity and the majority did not meet dietary guidelines for fruit and vegetable intake. There was low nutritional knowledge, particularly on healthy food choices and diet-disease relationships. Tobacco use and exposure and harmful use of alcohol were highly prevalent with male participants engaging in more substance use than females. An alarming pattern of early initiation of high-risk behaviours including underage and binge drinking, smoking and experimentation with cannabis emerged in the youngest age group. A detailed interpretation of these findings, comparisons to the general population and previous findings in PLHIV as well as implications for integrated prevention are discussed below.

\section{$76 \%$ of participants were female}

More than two-thirds of our respondents were female which is consistent with national laboratory data for adolescents in HIV care [136]. This reflects the gendered nature of the HIV epidemic in South Africa, where almost a quarter of all new HIV infections occur in young women aged 15-24 years [137], and much higher rates of health care-seeking among young women compared to young men $[42,138]$.

\section{4\% of AYLHIV were multidimensionally poor}

This is higher than national estimates which indicate that $33,4 \%$ of young people aged 15-24 years are MPI poor [139]. Nationally $34 \%$ of young people aged 15-24 years were not in education, employment, or training (NEET) in 2019 [140]. An "idle" youth population, not only impacts on the social cohesion and safety of a community, but is also linked to uptake of risk behaviours [141]. Although the proportion of NEETs in this study was lower than the national average with $73 \%$ of respondents either in education or engaged in income-generating activities, they experienced other deprivations which may interact to exacerbate vulnerability to NCDs and poor mental health. The highest deprivation was in general health and functioning dimension; with $68 \%$ experiencing difficulties with general health and functioning, $35 \%$ lived in informal housing, and $38 \%$ reported experiencing severe food insecurity.

Previous studies in South Africa have demonstrated that HIV/AIDS-affected and infected youth face multiple deprivations of poverty [60,119], including poor educational outcomes [142], informal housing, lack of basic necessities like warm clothing, toiletries and school fees [143] and food insecurity [144]. Socioeconomic barriers such as poverty and food insecurity are commonly cited challenges for adolescents receiving HIV treatment and care in SSA [145]. This is concerning because socioeconomic factors like food insecurity impact adherence to ART and retention in HIV care [146], which has implications for viral suppression and chronic disease pathways $[64,147,148]$. These factors also have an impact on mental health and may increase susceptibility to alcohol and substance use [149-152]. 
Addressing this challenge requires a multi-sectoral approach for NCD prevention with appropriate social protection systems to meet the needs of the most vulnerable [143].

\section{$36 \%$ overweight or obese and $37 \%$ had abdominal obesity}

More than a third of our respondents were overweight or obese (36\%), with significantly more overweight and centrally obese females compared to males. Although our rates of overweight and obesity are slightly lower than prevalence rates for youth in the Western Cape (31.5\% overweight and $11.3 \%$ obese) [38], the rates for ALYHIV appear similar to obesity trends in the general population. A previous study in adult patients attending primary health care HIV-clinics in South Africa found that more than half of female patients were overweight or obese compared to $16 \%$ of male patients [153]. Obesity in PLHIV is well documented in high-income countries and is emerging as a major challenge in Africa [50] with numerous studies showing increased rates of obesity in PLHIV [153-158]. But few studies in Africa have reported on overweight and obesity levels in AYLHIV other than in the context of ART-associated dyslipidaemia $[129,159,160]$.

One study conducted among South African university students living with HIV (the majority aged 2025 years) found that $21 \%$ were overweight and $30 \%$ were obese [161]. Findings from the United States Adolescent Trials Network showed that more than $40 \%$ of behaviourally HIV-infected young women (1424 years) were overweight or obese [162] and approximately $36 \%$ of perinatally HIV-infected adolescents were overweight or obese [163]. Our results are consistent with these findings and confirm results from a folder review conducted in this same population that reported similar levels of overweight and obesity [66]. Overweight and obesity in AYLHIV may accelerate their lifetime risk of cardiovascular diseases, additional to the effects of HIV-infection and exposure to ART [162]. In our study, obesity co-occurred with hypertension $-35 \%$ of those with elevated blood pressure or hypertension were also overweight or obese. By screening for obesity, other related conditions which tend to cluster with obesity can also be detected. Closer monitoring of overweight and obesity profiles in young PLHIV in SSA is needed as they transition into adulthood, especially with prolonged exposure to ART regimens which are linked to obesity, altered glucose metabolism and dyslipidaemia [164].

In addition to BMI, we assessed central obesity using waist and hip circumference indicators. We found that $26 \%$ of our respondents with normal BMI had high WHR or WHtR, meeting criteria for central obesity. Another South African study in adults attending three HIV-clinics reported a high prevalence of central obesity, primarily in women - $45 \%$ (4\% in men) [161]. In contrast, in a study conducted in Brazil, only $2.5 \%$ of children and adolescents on ART were overweight or obese based on subscapular skinfold thickness and less than $1 \%$ had a high WC [165]. In that study, the authors highlight that they expected a higher prevalence of overweight and obesity, but this was likely influenced by parallel gains in height and weight observed in adolescents [165]. In a comparison of anthropometric measures for predicting cardiometabolic risk in HIV patients in Cameroon, Dimala et al found that markers of adiposity like WC, WHR and WHtR are better than BMI at identifying HIV/AIDS patients with increased cardiometabolic risk [166]. 
The WHO and the International Diabetes Federation (IDF) recommend monitoring changes in waist circumference in addition to measuring $\mathrm{BMI}$, as this can provide an estimate of increased abdominal fat even without a change in BMI, particularly in HIV-positive populations on ARV medication and in female patients who have a higher prevalence of obesity $[79,167]$. Waist circumference and WHtR have been shown to be better predictors of cardiovascular disease risk factors in children and adolescents than BMI [168]. Waist-to-height ratio has the additional merit of not being dependent on age, sex or ethnicity, as the standard cut-off value of 0.5 is indicative of an increased cardiometabolic risk universally [107]. These findings underline the importance of anthropometry beyond BMI, especially in females. Anthropometric measurements and calculations are non-invasive, low-cost and easy-to-use interventions that can be used in primary care to identify AYLHIV who are at increased cardiometabolic risk.

\section{High levels of depression and psychological distress}

Almost half our participants reported symptoms of psychological distress, compared to less than a quarter of young people nationally [21]. Only $11 \%$ of those with mild, moderate or severe psychological distress reported previously being diagnosed with a mental health condition. Mental health conditions are prevalent in AYLHIV in both high-income and resource-limited settings [169]. Our results are generally consistent with prevalence rates of depression among children and adolescents living with HIV from other African countries which ranged from 18.9\% in Malawi [67], 25\% in Rwanda [170], 27\% in Tanzania [171] and 51.2\% prevalence of psychological distress in Uganda [172]. These results are difficult to pool together due to non-uniformity in the methods used for mental health screening [169].

Significantly more female participants reported depressive symptoms compared to males (51\% compared to $19 \%$ ) which is in line with global statistics on depression [173]. After the age of 15 , girls and women are twice more likely to be depressed compared to boys and men [174]. Our findings show that only $17 \%$ of those identified as having significant depression via CESD-10 reported being previously diagnosed with anxiety or depression. In a previous retrospective review in the same population, mental health conditions were documented in less than $5 \%$ of folders reviewed [66]. These findings highlight that there is a missed opportunity for identifying youth with mental health problems before suboptimal ART adherence or other adverse HIV, NCD and mental health outcomes occur.

A recent study conducted amongst AYLHIV aged 9-19 years attending a primary care clinic in Johannesburg found that $8 \%$ screened positive for symptoms of depression which is much lower than our findings. However, $60 \%$ of those study participants were young adolescents aged $9-12$ years and almost all were perinatally-infected (92\%) [116]. Older adolescents in that study (aged 16-19) were more likely to screen positive for depression compared to younger adolescents, which is in line with our findings of higher depression scores in older adolescents and young adults. It has been documented that perinatally-infected adolescents may present with less psychological problems compared to behaviourally-infected adolescents $[119,175]$. But similar rates of mental health conditions have been reported in HIV-exposed but uninfected youth entering adolescence [176]. The use of inappropriate comparison groups in studies showing higher rates of mental health disorders in AYLHIV, makes it difficult to determine whether these impairments are in fact due to HIV-infection or other social 
confounding factors. In a recent study conducted in Soweto, South Africa, the authors found similar rates of mental illness in perinatally-infected and uninfected adolescents, suggesting that other contributing social factors prevalent in the community may override the effect of HIV, especially in the era of highly active antiretroviral treatment [177].

Mental health screening is crucial in HIV care due to multiple psychological vulnerabilities associated with living with HIV and high rates of suicide in PLHIV [178-180]. Recommendations calling for integration of mental health services into HIV care have been made for adults [181]. It is important that adolescents are not overlooked in this respect. A study conducted in Johannesburg found that a simple way of identifying youth struggling with mental health problems at primary care level is by asking them about their future aspirations. Those who do not feel like they have control of their future or do not have a dream for the future are more likely to have symptoms of depression, anxiety or PTSD requiring further support [114].

\section{Low nutritional knowledge, especially on healthy food choices}

Participants scored less than $40 \%$ on general nutrition knowledge questions and particularly had poor knowledge of healthy food choices and associations between diet and diseases. Only $10 \%$ recognised that eating thick-cut instead of thin or crinkle cut chips could help reduce the amount of fat in someone's diet and $22 \%$ correctly identified that eating less trans-fats can prevent heart disease. To our knowledge, this is the first study to assess nutrition knowledge in AYLHIV in Africa. A South African study in schoolgoing adolescents aged $15-18$ years found that $77.5 \%$ scored below average on diet and nutrition knowledge questions [182]. The poor knowledge on nutrition-related NCDs amongst AYLHIV in our study is concerning in a country undergoing nutritional transition [183]. Nutrition knowledge is strongly correlated with dietary intake and is needed for better dietary habits [97]. Although adolescents may lack autonomy in navigating their food environment, this life stage is characterised by increasing independence and as such they need to be informed about the importance of diet and how it can affect their current health status or future adult health [182]. Some practices like healthy cooking methods requiring steaming, roasting or baking can be adopted in their homes with cooperation of parents and caregivers [184]. With adequate knowledge, older adolescents in our study who ate more fast-food and more meals prepared outside the home, could be encouraged to make healthier food choices.

\section{High fast-food and SSB consumption, and low physical activity}

Almost three-quarters of AYLHIV did not eat fruit daily and almost half did not eat vegetables daily, falling below recommended dietary guidelines of eating at least five portions of fruit and vegetables daily in order to reduce the risk of NCDs [185]. Female participants ate less fruit and ate more fast-food, deepfried foods and foods with added sugar daily compared to males. Younger adolescents ate less fruits, vegetables and wholegrains compared to older age groups. 
More respondents, particularly females, consumed deep-fried foods and fast-foods daily compared to provincial estimates for youth in the Western Cape, while the proportion who consumed SSB daily was lower (29\% compared to $42 \%$ ) [20]. Our results are comparable to local estimates that one in five schoolgoing youth skip breakfast [38]. A recent study on fast food and carbonated soft drink consumption among adolescents aged 12-15 years in 44 LMICs (not including South Africa) found that $44 \%$ of adolescents consumed a carbonated soft drink at least once per day in the past month [186]. A metaanalysis on SSB intake found that individuals who consumed $1-2$ servings per day, had a $26 \%$ greater risk of developing type 2 diabetes, and a $20 \%$ greater risk of metabolic syndrome compared to those who did not consume SSB or had less than one serving/month [187]. In order to promote healthy diet, a multisectoral approach that promotes a healthy food environment is required. The South African government has made strides in promoting healthier food environments by implementing mandatory legislation for salt reduction in processed foods in June 2016 [188] and a tax on sugar-sweetened beverages in April 2018 [189]. These measures were introduced in efforts to reduce the prevalence of hypertension, obesity, NCDs and excess salt and sugar consumption [190]. More efforts are needed to translate these measures into action at a community and household level, especially amongst young girls who have higher prevalence of obesity which is likely to persist till adulthood without intervention.

Our results support findings of gender differences in physical activity levels among South African adolescents similar to global reports [191, 192]. Despite more than two-thirds of our respondents using active transport; either walking to and from school or work, fewer than one-third had sufficient levels of physical activity necessary to promote health and prevent chronic diseases. Almost half spent three or more hours per day of their leisure time sedentary. This is similar to physical inactivity levels reported in urban-based South African students [191]. Sedentary behaviour was higher than general population estimates which showed that $30 \%$ of youth watched TV or played computer games for over three hours per day [38] and higher than estimates from other LMICs which found that $27.0 \%$ of adolescents engaged in three or more hours of sedentary behaviour per day. Our results are consistent with those from a study in Brazil which found that $71 \%$ of ALHIV were sedentary with a higher proportion among girls [193]. Another study conducted in Botswana, found that youth living with HIV had significantly lower levels of daily PA compared to uninfected controls [129]. Similarly, a study in Brazil in 10-15-year-old perinatallyinfected adolescents and age-sex matched controls also found that participants living with HIV had lower physical activity scores compared to healthy peers [194]. Additional research is needed in this setting to explore the relationship between PA and HIV in adolescents. A study with an age-and sex-matched uninfected control group from the same community would help to elucidate whether this relationship exists in South Africa.

\section{Early initiation of high-risk behaviours (smoking, alcohol, drugs)}

The median age at smoking initiation of 16 years found in our study was similar to the national average of 15.8 years for youth aged $15-24$ years. However, our findings that $48 \%$ of male and $25 \%$ of female AYLHIV smoked daily or occasionally was much higher than national estimates for young people aged 
15-24 years reported in the 2016 Demographic and Health Survey (29\% of males and $5 \%$ of females [20]). Furthermore, almost half of those in the youngest age group reported at least occasional cigarette smoking in the past month and used more alternative tobacco products like water-pipes compared to older age groups. Young adolescents in South Africa increasingly use water-pipes (known as hookah pipes) which are often available without restriction $[195,196]$. Our results likely reflect trends in the Western Cape - the province with the highest prevalence of tobacco smoking in South Africa- where a quarter of school-going youth are current smokers [38] and the mean age of smoking initiation is significantly lower than the national average at 14.5 years [21]. There are only a few studies, most from high-income settings, that address the prevalence of smoking among AYLHIV [197-199]. These studies report higher rates of smoking among AYLHIV compared to the general population, particularly among those who were behaviourally-infected [200]. Our findings corroborate this and add to the limited literature from low and middle-income settings.

Smoking increases the vulnerability of PLHIV for adverse lung health and multiplies their risk of developing cardiovascular diseases compared to HIV-negative smokers [201-203]. Smoking often cooccurs with other health risk behaviours like alcohol consumption [204]. More than half (58\%) of current smokers in our study had also drunk alcohol in the preceding month. Our results also indicate higher rates of current alcohol consumption compared to national estimates for South African youth ( $41 \%$ versus 33\%) [38]. A few studies in SSA have reported higher occurrence of alcohol consumption in AYLHIV compared to HIV-negative adolescents [135]. Auvert et al reported that $29 \%$ of AYLHIV in a South African mining town drank alcohol at least once a month although this study was conducted almost 20 years ago [134]. Another study conducted in Zimbabwe reported that $5.6 \%$ of adolescent females living with HIV drank alcohol in past month, compared to $4.5 \%$ of HIV-negative females [133]. Several studies conducted in young people from other regions have reported increased alcohol and drug use especially among male AYLHIV [205]. Our results suggest that this may be the case, but further research is needed in South Africa and SSA to elucidate whether HIV infection is associated with increased alcohol consumption in young people living in settings like South Africa where heavy drinking is endemic [22].

Our respondents reported higher rates of binge drinking in the past month compared to estimates for the general population [20] (21\% of females and $37 \%$ of males in our study, compared to $5 \%$ and $21 \%$ nationally). Alarmingly, half of the underage respondents ( $<18$ years) were current drinkers and $55 \%$ of them engaged in recent binge drinking. Males reported significantly more use of illegal drugs or substances to-get-high compared to females. Previous studies on risk behaviour among AYLHIV in SSA report a high prevalence of alcohol and substance use behaviour, especially among males in late adolescence $[135,206]$. In a study conducted amongst young people aged $15-26$ years in the rural Eastern Cape province of South Africa, $4 \%$ of female AYLHIV reported problem alcohol drinking and $5 \%$ reported ever using drugs [131], whilst $31 \%$ of male AYLHIV reported problem drinking and $54 \%$ reported ever using drugs [132]. Alcohol and drug use did not differ from HIV-uninfected young people residing in the same setting [131]. 
The Western Cape has significant rates of stimulant use such as methamphetamine and cocaine compared to the rest of the country [207]. Approximately $5 \%$ of learners in the Western Cape have used methamphetamine within their lifetime [208]. Our findings may indicate a general underlying substance use problem in young people in this setting, not necessarily related to HIV, but which predisposes AYLHIV to more vulnerability. High rates of substance use and implications for brain development, particularly amongst males and younger adolescents, is concerning and warrants targeted intervention. Prevention and early intervention strategies aimed at harm reduction are needed that incorporate environmental factors beyond individual behaviour.

\section{Other NCD comorbidity}

Our blood pressure findings are consistent with findings from studies in general adolescents in urban South Africa which have reported hypertension prevalence rates ranging from 8-16\% [209] and elevated BP prevalence of 35\% [210]. Chatterton-Kirchmeier et al reported a significantly higher prevalence of elevated blood pressure in a cohort of HIV-infected, predominantly African-American adolescents and young adults in the US compared to healthy children [211]. Globally, studies involving HIV-infected adults have demonstrated higher hypertension prevalence than the general population [212] and hypertension has been found to be associated with ART $[213,214]$. One in five young adults (18-35 years) attending an HIV clinic in the same setting in Khayelitsha had comorbid hypertension [65]. There is no clear link in the literature between HIV infection and elevated BP in paediatric populations. Nevertheless, routine monitoring of blood pressure in HIV care, even in younger populations, is warranted in settings like South African with a high background prevalence of hypertension, in order to avert future disease. This is especially relevant in light of evidence that blood pressure trajectories in childhood and adolescence predict future elevated BP and cardiovascular risk in adulthood [209, 210, 215].

None of the $25 \%$ with self-reported diabetes symptoms in our study had an abnormal measured random blood glucose. In a cohort study of South African youth living with perinatally-acquired HIV, the authors found a high prevalence of insulin resistance but it did not differ from that in uninfected age-matched adolescents [128]. A systematic review and meta-analysis of African studies, recently reported that there was no statistically significant association between HIV infection or ART exposure and type 2 diabetes (T2DM) prevalence in adults [216]. This is in contrast with study findings from European and North American settings that have shown a higher prevalence of T2DM in HIV-infected adults particularly those on ART [217-219]. However, the cumulative incidence of T2DM in patients with HIV across Africa was higher than international incidence data for HIV-infected individuals. The International Diabetes Federation estimates that $60 \%$ of people with diabetes in Africa are undiagnosed [220] suggesting that T2DM might be a major, underdiagnosed public health problem in African populations in general due to the presence of traditional risk factors.

\section{Strengths and Limitations}

Our study adds to the limited evidence base on NCD prevalence and risk factors in AYLHIV in subSaharan Africa. To our knowledge, only four other studies in sub-Saharan Africa have investigated 
modifiable NCD risk factors besides alcohol and substance use in AYLHIV [127, 129, 161, 221]. While our study provides novel findings for the sub-Saharan African context, the findings should be interpreted within the following limitations. Firstly, participants were recruited using convenience sampling from healthcare facilities in a peri-urban setting. Second, there was an almost $50 \%$ non-response rate. The low response rate and lack of random sampling may limit the generalizability of our findings, however sampling from six different facilities across all substructures in the City of Cape Town, proportional to the total numbers of AYLHIV within each substructure) mitigated unmeasured facility-specific effects. Limited participation and low response rates are a major challenge in adolescent research due to the complexities of enrolment and consent procedures [222, 223]. Although we recruited younger adolescents, requiring parental consent may have led to participant bias as the majority enrolled were older adolescents and young adults (aged $\geq 18$ years) who could provide independent consent to participate. We therefore conducted an age-stratified analysis of participant characteristics.

Although we were not statistically powered to detect differences by gender or age groups, some clear differences emerged particularly gender differences in physical activity, obesity levels and mental health which have been previously documented. Striking differences in high-risk behaviours were also identified in the younger age group. Due to the cross-sectional design, we were unable to establish temporality and whether NCD risk factors and risky behaviours preceded an HIV diagnosis amongst behaviourallyinfected youth. Our study did not include a control group of HIV-uninfected adolescents, neither did we differentiate between perinatally and behaviourally-infected adolescents who may have very different risk profiles $[57,119]$. Nevertheless, since young people come from the same communities and access the same HIV services, irrespective of mode of transmission, interventions targeting risk factors generally may be more effective.

We used subjective recall methods of measuring physical activity which may be prone to over-reporting $[224,225]$, recall bias and cultural misinterpretation [226, 227]. However, self-report methods like the IPAQ have acceptable validity and are most widely used to measure physical activity in PLHIV [51]. The use of point-of-care random blood glucose testing may have underestimated diabetes risk, however, POC methods are better suited for community screening of diabetes, have high specificity (90\%) and provide reliable and immediate results $[228,229]$. Similarly, the mental health tools used are screening tools- a diagnosis of depression or anxiety was not confirmed using these tools. But they are appropriate for case-finding in primary care and have been validated in HIV-positive populations in South Africa [103, 230]. Despite using a self-administered questionnaire, there remains a possibility that social desirability or other reporting biases may affect reports of mental health and substance use, with potential for underreporting. To minimise this risk, we used tablet computers for data collection which have been found to reduce reporting bias on sensitive questions [231, 232]. Moreover, the reported rates were still significant and if underreported, warrant further attention. Despite these limitations, this study is an important contribution to the limited literature in sub-Saharan Africa.

\section{Conclusions}


This paper contributes to a key gap in the literature on NCD risk in AYLHIV in SSA. The findings highlight the existence of cardiometabolic risk factors (obesity, abdominal obesity, hypertension, physical inactivity, unhealthy diet), smoking, excessive drinking, and mental health problems in this vulnerable population, highlighting the need for NCD screening and integrated primary and secondary prevention.

NCDs and their ensuing burden of disability and premature mortality are costly to health systems and to wider societal development. Beyond primary care, the complex and interlinked social, economic and environmental factors that influence these behaviours highlight the importance of intersectoral action for disease prevention. It is therefore necessary to go beyond the healthcare sector to address the root causes and multiple deprivations that increase the risk of NCDs and ill-health, and to support equitable access to the necessary physical and social infrastructure required to make the healthy choice the easy choice. Upstream strategies that incorporate the basic living conditions and environments in which young people live are necessary to ensure well-being and interrupt disease pathways. More studies are needed to assess risk factors at a broader socio-ecological level and explore inter-relationships between NCD/HIV comorbidity and the environment in order to identify effective and sustainable risk-reduction interventions.

\section{Abbreviations}

AYLHIV

Adolescents and youth living with HIV;

$\mathrm{BMI}$

Body mass index;

BP

Blood pressure;

CESD-10

Centre for Epidemiological Studies Short Depression Scale;

CMNN

Communicable, Maternal, Neonatal, and Nutritional;

FFQ

Food frequency questionnaire;

GNKQ

General Nutrition Knowledge Questionnaire;

HFIAS

Household Food Insecurity Access Scale;

IPAQ

International Physical Activity Questionnaire;

K10

Kessler Psychological Distress Scale;

LMIC

Low- and middle-income countries; 
MET

Metabolic equivalent of task;

NCD

Non-communicable disease;

NEET

Neither in education, employment or training;

PA

Physical activity;

PLHIV

People living with HIV;

SSA

Sub-Saharan Africa;

SSB

Sugar-sweetened beverages;

SANHANES

South African National Health and Nutrition Examination Survey;

WC

Waist circumference;

WHR

Waist-hip ratio;

WHtR

Waist-to-height ratio;

YMPI

Youth Multidimensional Poverty Index;

YRBS

Youth Risk Behaviour Survey;

\section{References}

1. Naghavi M. Global, regional, and national burden of suicide mortality 1990 to 2016: systematic analysis for the Global Burden of Disease Study 2016. BMJ. 2019:194.

2. Vos T, Abajobir AA, Abate KH, et al. Global, regional, and national incidence, prevalence, and years lived with disability for 328 diseases and injuries for 195 countries, 1990-2016: a systematic analysis for the Global Burden of Disease Study 2016. Lancet. 2017;390(10100):1211-1259.

3. Naghavi M, Abajobir AA, Abbafati C, et al. Global, regional, and national age-sex specific mortality for 264 causes of death, 1980-2016: a systematic analysis for the Global Burden of Disease Study 2016. Lancet. 2017;390(10100):1151-1210.

4. World Health Organization. Noncommunicable diseases progress monitor 2020. Geneva: World Health Organization; 10 February 2020 2020. 978-92-4-000049-0. 
5. Reitsma MB, Fullman N, Ng M, et al. Smoking prevalence and attributable disease burden in 195 countries and territories, 1990-2015: a systematic analysis from the Global Burden of Disease Study 2015. Lancet. 2017;389(10082):1885-1906.

6. Forouzanfar MH, Afshin A, Alexander LT, et al. Global, regional, and national comparative risk assessment of 79 behavioural, environmental and occupational, and metabolic risks or clusters of risks, 1990-2015: a systematic analysis for the Global Burden of Disease Study 2015. Lancet. 2016;388(10053):1659-1724.

7. Degenhardt $L$, Charlson F, Ferrari $A$, et al. The global burden of disease attributable to alcohol and drug use in 195 countries and territories, 1990-2016: a systematic analysis for the Global Burden of Disease Study 2016. Lancet Psychiatry. 2018;5(12):987-1012.

8. Griswold MG, Fullman N, Hawley C, et al. Alcohol use and burden for 195 countries and territories, 1990-2016: a systematic analysis for the Global Burden of Disease Study 2016. Lancet. 2018;392(10152):1015-1035.

9. Lee IM, Shiroma EJ, Lobelo F, Puska P, Blair SN, Katzmarzyk PT. Effect of physical inactivity on major non-communicable diseases worldwide: an analysis of burden of disease and life expectancy. Lancet. 2012;380(9838):219-229.

10. Guthold R, Stevens GA, Riley LM, Bull FC. Worldwide trends in insufficient physical activity from 2001 to 2016: a pooled analysis of 358 population-based surveys with 1.9 million participants. Lancet Glob. Health. 2018;6(10):e1077-e1086.

11. Afshin A, Sur PJ, Fay KA, et al. Health effects of dietary risks in 195 countries, 1990-2017: a systematic analysis for the Global Burden of Disease Study 2017. Lancet. 2019;393(10184):19581972.

12. James SL, Abate $\mathrm{D}$, Abate $\mathrm{KH}$, et al. Global, regional, and national incidence, prevalence, and years lived with disability for 354 diseases and injuries for 195 countries and territories, 1990-2017: a systematic analysis for the Global Burden of Disease Study 2017. Lancet. 2018;392(10159):17891858.

13. Stein DJ, Benjet C, Gureje O, et al. Integrating mental health with other non-communicable diseases. BMJ. 2019:I295.

14. World Health Organization. Noncommunicable diseases. 2018; Media centre, Fact sheet Available at: https://www.who.int/news-room/fact-sheets/detail/noncommunicable-diseases. Accessed 21 April 2020, 2020.

15. Gouda HN, Charlson F, Sorsdahl K, et al. Burden of non-communicable diseases in sub-Saharan Africa, 1990-2017: results from the Global Burden of Disease Study 2017. Lancet Glob. Health. 2019;7(10):e1375-e1387.

16. Pillay-Van Wyk V, Msemburi W, Laubscher R, et al. Mortality trends and differentials in South Africa from 1997 to 2012: second National Burden of Disease Study. Lancet Glob. Health. 2016;4(9):e642e653. 
17. Remais JV, Zeng G, Li G, Tian L, Engelgau MM. Convergence of non-communicable and infectious diseases in low-and middle-income countries. Int J Epidemiol. 2013;42(1):221-227.

18. World Health Organization. Report on the status of major health risk factors for noncommunicable diseases: WHO African Region, 2015. 2016.

19. Kengne AP. Trends in obesity and diabetes across Africa from 1980 to 2014: an analysis of pooled population-based studies. Int J Epidemiol. Oct 1 2017;46(5):1421-1432.

20. National Department of Health (NDoH); Statistics South Africa (Stats SA); South African Medical Research Council (SAMRC); ICF. South Africa demographic and health survey 2016.

21. Shisana O. The South African National Health and Nutrition Examination Survey: SANHANES-1: HSRC press; 2013.

22. World Health Organization. Global status report on alcohol and health 2018: World Health Organization; 2019.

23. Bennett JE, Stevens GA, Mathers CD, et al. NCD Countdown 2030: worldwide trends in noncommunicable disease mortality and progress towards Sustainable Development Goal target 3.4. Lancet. 2018;392(10152):1072-1088.

24. Steinberg L, Morris AS. Adolescent development. J Cogn Educ Psychol. 2001;2(1):55-87.

25. World Health Organization. The second decade: improving adolescent health and development. Geneva: World Health Organization;2001.

26. World Health Organization. Orientation programme on adolescent health for health care providers. 2006.

27. Whitaker RC, Wright JA, Pepe MS, Seidel KD, Dietz WH. Predicting obesity in young adulthood from childhood and parental obesity. N. Engl. J. Med.1997;337(13):869-873.

28. Daniels SR. The consequences of childhood overweight and obesity. Future Child. 2006;16(1):47-67.

29. Freedman DS, Dietz WH, Srinivasan SR, Berenson GS. The relation of overweight to cardiovascular risk factors among children and adolescents: the Bogalusa Heart Study. Pediatrics. 1999;103(6):1175-1182.

30. Freedman DS, Khan LK, Dietz WH, Srinivasan SR, Berenson GSJP. Relationship of childhood obesity to coronary heart disease risk factors in adulthood: the Bogalusa Heart Study. 2001;108(3):712-718.

31. Reilly JJ, Methven E, McDowell ZC, et al. Health consequences of obesity. Health consequences of obesity. Arch. Dis. Child. 2003 Sep 1;88(9):748-52.

32. Kessler RC, Berglund P, Demler O, Jin R, Merikangas KR, Walters EE. Lifetime prevalence and age-ofonset distributions of DSM-IV disorders in the National Comorbidity Survey Replication. Arch Gen Psychiatry. 2005;62(6):593-602.

33. Sawyer SM, Afifi RA, Bearinger LH, et al. Adolescence: a foundation for future health. Lancet. Apr 28 2012;379(9826):1630-1640.

34. Azzopardi P. Adolescent health comes of age. Lancet. 2012;379(9826):1583-1584. 
35. Statistics South Africa. Statistical release P0302: mid-year population estimates. Available at: https://www.statssa.gov.za/publications/P0302/P03022016.pdf. Accessed June 04, 2020.

36. $\mathrm{Ng} \mathrm{M}$, Fleming $T$, Robinson $\mathrm{M}$, et al. Global, regional, and national prevalence of overweight and obesity in children and adults during 1980-2013: a systematic analysis for the Global Burden of Disease Study 2013. Lancet. 2014 Aug 30;384(9945):766-81.

37. Rossouw HA, Grant CC, Viljoen M. Overweight and obesity in children and adolescents: The South African problem. S. Afr. J. Sci. 2012;108(5-6):31-37.

38. Reddy S, James S, Sewpaul R, et al. Umthente uhlaba usamila: the 3rd South African national youth risk behaviour survey 2011. 2013.

39. Otwombe K, Dietrich J, Laher F, et al. Health-seeking behaviours by gender among adolescents in Soweto, South Africa. Glob Health Action. 2015;8(1):25670.

40. UNAIDS. 2016; Available at: http://aidsinfo.unaids.org/. Accessed 15 June 2017, 2017.

41. Sohn AH, Hazra R. The changing epidemiology of the global paediatric HIV epidemic: keeping track of perinatally HIV-infected adolescents. J Int AIDS Soc. 2013;16:18555.

42. Dellar RC, Dlamini S, Karim QA. Adolescent girls and young women: key populations for HIV epidemic control. J Int AIDS Soc. 2015;18(2(Suppl 1)):19408.

43. Battles HB, Wiener LS. From adolescence through young adulthood: psychosocial adjustment associated with long-term survival of HIV. J Adolesc Health. 2002;30(3):161-168.

44. Achwoka D, Waruru A, Chen TH, et al. Noncommunicable disease burden among HIV patients in care: a national retrospective longitudinal analysis of HIV-treatment outcomes in Kenya, 2003-2013. BMC Public Health. Apr 3 2019;19(1):372.

45. Miller TL, Borkowsky W, DiMeglio LA, et al. Metabolic abnormalities and viral replication are associated with biomarkers of vascular dysfunction in HIV-infected children. HIV Med. 2012;13(5):264-275.

46. Deeks SG. HIV infection, inflammation, immunosenescence, and aging. Annu. Rev. Med. 2011;62:141.

47. Agwu AL, Fairlie L. Antiretroviral treatment, management challenges and outcomes in perinatally HIVinfected adolescents. J Int AIDS Soc. 2013;16(1).

48. Eckard AR, Mora S. Bone health in HIV-infected children and adolescents. Curr Opin HIV AIDS. 2016;11(3):294-300.

49. Eckard AR, Fowler SL, Haston JC, Dixon TC. Complications of Treatment in Youth with HIV. Curr. HIV/AIDS Rep. 2016;13(4):1-8.

50. Deeks SG, Lewin SR, Havlir DV. The End of AIDS: HIV Infection as a Chronic Disease. Lancet. 10/23 2013;382(9903):1525-1533.

51. Vancampfort D, Mugisha J, De Hert M, et al. Global physical activity levels among people living with HIV: a systematic review and meta-analysis. Disabil. Rehabil. 2018;40(4):388-397. 
52. Reynolds NR. Cigarette smoking and HIV: more evidence for action. AIDS Educ Prev. Jun 2009;21(3 Suppl):106-121.

53. Petrosillo N, Cicalini S. Smoking and HIV: time for a change? BMC Med. 2013;11(1):16.

54. Scott-Sheldon LAJ, Walstrom P, Carey KB, Johnson BT, Carey MP. Alcohol Use and Sexual Risk Behaviors among Individuals Infected with HIV: A Systematic Review and Meta-Analysis 2012 to Early 2013. Curr. HIV/AIDS Rep. 2013;10(4):314-323.

55. Kalichman S, Mathews C, Banas E, Kalichman M. Alcohol-related intentional nonadherence to antiretroviral therapy among people living with HIV, Cape Town, South Africa. AIDS Care. 2019;31(8):951-957.

56. Kader R, Govender R, Seedat S, Koch JR, Parry C. Understanding the Impact of Hazardous and Harmful Use of Alcohol and/or Other Drugs on ARV Adherence and Disease Progression. PLoS One. 2015;10(5):e0125088.

57. Mellins CA, Malee KM. Understanding the mental health of youth living with perinatal HIV infection: lessons learned and current challenges. J Int AIDS Soc. 2013;16(1).

58. Betancourt TS, Rubin-Smith JE, Beardslee WR, Stulac SN, Fayida I, Safren S. Understanding locally, culturally, and contextually relevant mental health problems among Rwandan children and adolescents affected by HIV/AIDS. AIDS Care. 2011;23(4):401-412.

59. Langhaug LF, Pascoe SJ, Mavhu W, et al. High prevalence of affective disorders among adolescents living in Rural Zimbabwe. J Community Health. 2010;35(4):355-364.

60. Cluver L, Gardner F, Operario D. Poverty and psychological health among AIDS-orphaned children in Cape Town, South Africa. AIDS Care. 2009;21(6):732-741.

61. Murphy DA, Durako SJ, Moscicki A-B, et al. No change in health risk behaviors over time among HIV infected adolescents in care: role of psychological distress. J Adolesc Health. 2001;29(3):57-63.

62. Mellins CA, Elkington KS, Bauermeister JA, et al. Sexual and Drug Use Behavior in Perinatally HIVInfected Youth: Mental Health and Family Influences. J Am Acad Child Adolesc Psychiatry. 2009;48(8):810-819.

63. Anand P, Springer SA, Copenhaver MM, Altice FL. Neurocognitive Impairment and HIV Risk Factors: A Reciprocal Relationship. AIDS Behav. 2010;14(6):1213-1226.

64. Innes S, Patel K. Noncommunicable diseases in adolescents with perinatally acquired HIV-1 infection in high-income and low-income settings. Curr Opin HIV AIDS. May 2018;13(3):187-195.

65. Oni T, Youngblood E, Boulle A, McGrath N, Wilkinson RJ, Levitt NS. Patterns of HIV, TB, and noncommunicable disease multi-morbidity in peri-urban South Africa- a cross sectional study. BMC Infect. Dis. 2015;15:20.

66. Kamkuemah M, Gausi B, Oni T. Missed opportunities for NCD multimorbidity prevention in adolescents and youth living with HIV in urban South Africa. BMC Public Health. 2020;20(821).

67. Kim MH, Mazenga AC, Yu X, et al. Factors associated with depression among adolescents living with HIV in Malawi. BMC Psychiatry. Oct 26 2015;15:264. 
68. Senyonyi RM, Underwood LA, Suarez E, Musisi S, Grande TL. Cognitive behavioral therapy group intervention for HIV transmission risk behavior in perinatally infected adolescents. Health. 2012;04(12):1334-1345.

69. Mbalinda SN, Kiwanuka N, Kaye DK, Eriksson LE. Reproductive health and lifestyle factors associated with health-related quality of life among perinatally HIV-infected adolescents in Uganda. Health Qual. Life Outcomes. 2015;13(1).

70. Western Cape Government 2017 Socio-economic Profile: City of Cape Town. Department of Social Development, ed. Cape Town. 2017.

71. Western Cape Government. Annual Performance Plan 2016 - 2017. Health Department, ed. Cape Town. 2016.

72. Republic of South Africa National Department of Health. The 2015 national antenatal sentinel HIV \& syphilis survey, South Africa. 2017.

73. Republic of South Africa National Department of Health. The 2012 National Antenatal Sentinel HIV \& Herpes Simplex Type-2 Prevalence Survey in South Africa. 2013

74. Cape Metro Health District. CAPE METRO DISTRICT HEALTH PLAN 2018/19 - 2020/21. District Management Team, ed. Cape Town. 2018.

75. Statistics South Africa. Mortality and Causes of Death in South Africa, 2016: Findings from Death Notification: Statistics South Africa; 2018.

76. Western Cape Government. Annual Performance Plan 2019. Health Department, ed. Cape Town. 2019.

77. City of Cape Town. City of Cape Town - 2011 Census - Cape Flats Planning District. Cape Town. 2013.

78. City of Cape Town. 2016 Socio-economic Profile: City of Cape Town. 2016.

79. World Health Organization. Waist circumference and waist-hip ratio: report of a WHO expert consultation, Geneva, 8-11 December 2008. 2011.

80. Miller MR, Hankinson J, Brusasco V, et al. Standardisation of spirometry. Eur Respir J. 2005;26(2):319-338.

81. Klein H. Adolescence, youth, and young adulthood: Rethinking current conceptualizations of life stage. Youth Soc. 1990;21(4):446-471.

82. Statistics South Africa. Census Questionnaire. 2011; Available at: http://www.statssa.gov.za/? page_id=3852. Accessed 29 April, 2020.

83. Alkire S, Santos ME. Acute multidimensional poverty: A new index for developing countries. 2010.

84. Frame E, De Lannoy A, Leibbrandt M. Measuring multidimensional poverty among youth in South Africa at the sub-national level. 2016.

85. Alkire S, Conconi A, Pinilla-Roncancio M, Vaz A. How to Build a National Multidimensional Poverty Index (MPI): Using the MPI to inform the SDGs.2018 
86. Ballard T, Coates J, Swindale A, Deitchler M. Household hunger scale: indicator definition and measurement guide. Washington, DC: Food and Nutrition Technical Assistance II Project, FHI. 2011 Aug;360.

87. Coates J, Swindale A, Bilinsky P. Household Food Insecurity Access Scale (HFIAS) for measurement of food access: indicator guide: version 3. 2007.

88. Craig C, Marshall A, Sjostrom M, et al. International Physical Activity Questionnaire-Short Form. 2017.

89. Craig CL, Marshall AL, Sjöström M, et al. International physical activity questionnaire: 12-country reliability and validity. Med Sci Sports Exerc. 2003;35(8):1381-1395.

90. Ainsworth BE, Haskell WL, Whitt MC, et al. Compendium of physical activities: an update of activity codes and MET intensities. Med Sci Sports Exerc. 2000;32(9):S498-S516.

91. IPAQ Research Committee. Guidelines for the data processing and analysis of the International Physical Activity Questionnaire. 2005.

92. World Health Organization. WHO Global recommendations on physical activity for health. Geneva: World Health Organization; 2011. Geneva, Switzerland: World Health Organization; 2011.

93. Guthold R, Cowan MJ, Autenrieth CS, Kann L, Riley LM. Physical activity and sedentary behavior among schoolchildren: a 34-country comparison.

J Pediatr. 2010;157(1):43-49. e41.

94. Katzmarzyk PT, Barreira TV, Broyles ST, et al. The international study of childhood obesity, lifestyle and the environment (ISCOLE): design and methods. BMC Public Health. 2013;13(1):900.

95. Saloheimo T, González SA, Erkkola M, et al. The reliability and validity of a short food frequency questionnaire among 9-11-year olds: a multinational study on three middle-income and high-income countries. Int J Obes (Lond) 2015;5(S2):S22-S28.

96. Kliemann N, Wardle J, Johnson F, Croker H. Reliability and validity of a revised version of the General Nutrition Knowledge Questionnaire. Eur J Clin Nutr. 2016;70(10):1174-1180.

97. Wardle J, Parmenter K, Waller J. Nutrition knowledge and food intake. Appetite. 2000;34(3):269-275.

98. Karl Peltzer K-N. Nutrition knowledge among a sample of urban black and white South Africans. South Afr J Clin Nutr. 2004;17(1):24-31.

99. Andresen EM, Malmgren JA, Carter WB, Patrick DL. Screening for depression in well older adults: Evaluation of a short form of the CES-D. Am J Prev Med. 1994;10(2):77-84.

100. Radloff LS. The CES-D scale: A self-report depression scale for research in the general population. Appl Psychol Meas. 1977;1(3):385-401.

101. Kessler RC, Barker PR, Colpe LJ, et al. Screening for serious mental illness in the general population. Arch. Gen. Psychiatry. 2003;60(2):184-189.

102. Myer L, Smit J, Roux LL, Parker S, Stein DJ, Seedat S. Common mental disorders among HIV-infected individuals in South Africa: prevalence, predictors, and validation of brief psychiatric rating scales. AIDS patient care and STDs. 2008;22(2):147-158. 
103. Spies G, Kader K, Kidd M, et al. Validity of the K-10 in detecting DSM-IV-defined depression and anxiety disorders among HIV-infected individuals. AIDS Care. 2009;21(9):1163-1168.

104. Radloff LS. The use of the Center for Epidemiologic Studies Depression Scale in adolescents and young adults. $J$ Youth Adolesc. 1991;20(2):149-166.

105. Andrews G, Slade T. Interpreting scores on the Kessler psychological distress scale (K10). Aust N Z J Public Health. 2001;25(6):494-497.

106. Barlow SE. Expert Committee Recommendations Regarding the Prevention, Assessment, and Treatment of Child and Adolescent Overweight and Obesity: Summary Report. Pediatrics. 2007;120(Supplement 4):S164-S192.

107. Browning LM, Hsieh SD, Ashwell M. A systematic review of waist-to-height ratio as a screening tool for the prediction of cardiovascular disease and diabetes: 0.5 could be a suitable global boundary value. Nutr Res Rev. 2010;23(2):247-269.

108. Seedat Y, Rayner B, Veriava Y. South African hypertension practice guideline 2014. Cardiovasc J Afr. 2014;25(6):288.

109. Smit M, Olney J, Ford NP, et al. The growing burden of noncommunicable disease among persons living with HIV in Zimbabwe. AIDS. Mar 27 2018;32(6):773-782.

110. Meghji J, Nadeau G, Davis KJ, et al. Noncommunicable Lung Disease in Sub-Saharan Africa. A Community-based Cross-Sectional Study of Adults in Urban Malawi. Am J Respir Crit Care Med. Jul 1 2016;194(1):67-76.

111. Sobieszczyk ME, Werner L, Mlisana K, et al. Metabolic Syndrome After HIV Acquisition in South African Women. J Acquir Immune Defic Syndr. Dec 1 2016;73(4):438-445.

112. Patel $P$, Rose $C E$, Collins PY, et al. Noncommunicable diseases among HIV-infected persons in lowincome and middle-income countries: a systematic review and meta-analysis. AIDS (London, England). 2018;32(Suppl 1):S5.

113. Wright J, Lubben F, Mkandawire MB. Young Malawians on the interaction between mental health and HIV/AIDS. Afr J AIDS Res. Nov 2007;6(3):297-304.

114. Woollett $\mathrm{N}$, Cluver $\mathrm{L}$, Bandeira $\mathrm{M}$, Brahmbhatt $\mathrm{H}$. Identifying risks for mental health problems in HIV positive adolescents accessing HIV treatment in Johannesburg. $J$ Child Adolesc Ment Health. May 2017;29(1):11-26.

115. Willis N, Mavhu W, Wogrin C, Mutsinze A, Kagee A. Understanding the experience and manifestation of depression in adolescents living with HIV in Harare, Zimbabwe. PLoS One. 2018;13(1):e0190423.

116. West N, Schwartz S, Mudavanhu M, et al. Mental health in South African adolescents living with HIV. AIDS Care. Jan 2019;31(1):117-124.

117. Webster KD, de Bruyn MM, Zalwango SK, et al. Caregiver socioemotional health as a determinant of child well-being in school-aged and adolescent Ugandan children with and without perinatal HIV exposure. Trop Med Int Health. May 2019;24(5):608-619. 
118. Taylor Salisbury T, Kinyanda E, Levin J, et al. Clinical correlates and adverse outcomes of ADHD, disruptive behavior disorder and their co-occurrence among children and adolescents with HIV in Uganda. AIDS Care. Mar 19 2020:1-9.

119. Sherr L, Cluver LD, Toska E, He E. Differing psychological vulnerabilities among behaviourally and perinatally HIV infected adolescents in South Africa - implications for targeted health service provision. AIDS Care. Jun 2018;30(sup2):92-101.

120. Sam-Agudu NA, Pharr JR, Bruno T, et al. Adolescent Coordinated Transition (ACT) to improve health outcomes among young people living with HIV in Nigeria: study protocol for a randomized controlled trial. Trials. Dec 14 2017;18(1):595.

121. Ramaiya MK, Sullivan KA, K OD, et al. A Qualitative Exploration of the Mental Health and Psychosocial Contexts of HIV-Positive Adolescents in Tanzania. PLoS One. 2016;11(11):e0165936.

122. Desai SR, Nair A, Rylance J, et al. Human immunodeficiency virus-associated chronic lung disease in children and adolescents in Zimbabwe: chest radiographic and high-resolution computed tomographic findings. Clin Infect Dis. 2018 Jan 6;66(2):274-81.

123. Githinji LN, Gray DM, Hlengwa S, Myer L, Zar HJ. Lung Function in South African Adolescents Infected Perinatally with HIV and Treated Long-Term with Antiretroviral Therapy. Ann. Am. Thorac. Soc. 2017;14(5):722-729.

124. Rylance J, McHugh G, Metcalfe J, et al. Chronic lung disease in HIV-infected children established on antiretroviral therapy. AIDS. Nov 28 2016;30(18):2795-2803.

125. Mwalukomo T, Rylance SJ, Webb EL, et al. Clinical Characteristics and Lung Function in Older Children Vertically Infected With Human Immunodeficiency Virus in Malawi. J. Pediatric Infect. Dis. Soc. 2016;5(2):161-169.

126. Ferrand RA, Desai SR, Hopkins $C$, et al. Chronic lung disease in adolescents with delayed diagnosis of vertically acquired HIV infection. Clin Infect Dis. Jul 2012;55(1):145-152.

127. Ssewanyana D, Newton CR, van Baar A, et al. Beyond Their HIV Status: the Occurrence of Multiple Health Risk Behavior Among Adolescents from a Rural Setting of Sub-Saharan Africa. Int J Behav Med. Mar 252020.

128. Frigati LJ, Jao J, Mahtab S, et al. Insulin Resistance in South African Youth Living with Perinatally Acquired HIV Receiving Antiretroviral Therapy. AIDS Res. Hum. Retroviruses. 2019;35(1):56-62.

129. Malete L, Tladi DM, Etnier JL, Makhanda J, Anabwani GM. Examining psychosocial correlates of physical activity and sedentary behavior in youth with and without HIV. PLOS One. 2019;14(12):e0225890.

130. Gleason RL, Jr., Caulk AW, Seifu D, et al. Efavirenz and ritonavir-boosted lopinavir use exhibited elevated markers of atherosclerosis across age groups in people living with HIV in Ethiopia. $J$ Biomech. Sep 6 2016;49(13):2584-2592.

131. Jewkes R, Dunkle K, Nduna M, et al. Factors associated with HIV sero-status in young rural South African women: connections between intimate partner violence and HIV. Int J Epidemiol. 2006;35(6):1461-1468. 
132. Jewkes R, Dunkle K, Nduna M, et al. Factors associated with HIV sero-positivity in young, rural South African men. Int J Epidemiol. 2006;35(6):1455-1460.

133. Gavin L, Galavotti C, Dube H, et al. Factors associated with HIV infection in adolescent females in Zimbabwe. J Adolesc Health. 2006;39(4):596. e511-596. e518.

134. Auvert B, Ballard R, Campbell C, et al. HIV infection among youth in a South African mining town is associated with herpes simplex virus-2 seropositivity and sexual behaviour. AIDS. 2001;15(7):885898.

135. Ssewanyana D, Mwangala PN, Van Baar A, Newton CR, Abubakar A. Health risk behaviour among adolescents living with HIV in sub-Saharan Africa: a systematic review and meta-analysis. BioMed Res. Int. 2018;2018.

136. Maskew M, Bor J, MacLeod W, Carmona S, Sherman GG, Fox MP. Adolescent HIV treatment in South Africa's national HIV programme: a retrospective cohort study. Lancet HIV. 2019;6(11):e760-e768.

137. Shisana O, Rehle T, Simbayi LC, et al. South African national HIV prevalence, incidence and behaviour survey, 2012. 2014.

138. Shisana O, Rice K, Zungu N, Zuma K. Gender and poverty in South Africa in the era of HIV/AIDS: a quantitative study. J Womens Health (LarChmt). Jan 2010;19(1):39-46.

139. Statistics South Africa. Living Conditions of Households in South Africa 2014/2015. In: Africa SS, ed. Pretoria2017.

140. Statistics South Africa. Quarterly Labour Force Survey, Quarter 1: 2019: Statistics South Africa Pretoria; 2019.

141. Hall K, De Lannoy A. Children's access to education. ChildGauge. 2019.

142. Orkin M, Boyes ME, Cluver LD, Zhang Y. Pathways to poor educational outcomes for HIV/AIDSaffected youth in South Africa. AIDS Care. 2014;26(3):343-350.

143. Cluver LD, Orkin FM, Campeau L, et al. Improving lives by accelerating progress towards the UN Sustainable Development Goals for adolescents living with HIV: a prospective cohort study. Lancet Child Adolesc. Health. Apr 2019;3(4):245-254.

144. Young S, Wheeler AC, McCoy SI, Weiser SD. A review of the role of food insecurity in adherence to care and treatment among adult and pediatric populations living with HIV and AIDS. AIDS Behav. 2014;18(5):505-515.

145. Mark D, Armstrong A, Andrade C, et al. HIV treatment and care services for adolescents: a situational analysis of 218 facilities in 23 sub-Saharan African countries. J Int AIDS Soc. 2017;20(0):21591.

146. Harrison S, Li X. Toward an enhanced understanding of psychological resilience for HIV youth populations. AIDS Care. 2018;30(sup4):1-4.

147. Agu CE, Uchendu IK, Nsonwu AC, Okwuosa CN, Achukwu PU. Prevalence and associated risk factors of peripheral artery disease in virologically suppressed HIV-infected individuals on antiretroviral therapy in Kwara state, Nigeria: a cross sectional study. BMC Public Health. Aug 20 2019;19(1):1143. 
148. Barai N, Monroe A, Lesko C, et al. The Association Between Changes in Alcohol Use and Changes in Antiretroviral Therapy Adherence and Viral Suppression Among Women Living with HIV. AIDS Behav. Jul 2017;21(7):1836-1845.

149. Cagle A, McGrath C, Richardson BA, et al. Alcohol use and immune reconstitution among HIVinfected patients on antiretroviral therapy in Nairobi, Kenya. AIDS Care. Sep 2017;29(9):1192-1197.

150. Morojele NK, Kekwaletswe CT, Nkosi S. Associations between alcohol use, other psychosocial factors, structural factors and antiretroviral therapy (ART) adherence among South African ART recipients. AIDS Behav. Mar 2014;18(3):519-524.

151. Shacham E, Agbebi A, Stamm K, Overton ET. Alcohol consumption is associated with poor health in HIV clinic patient population: a behavioral surveillance study. AIDS Behav. Jan 2011;15(1):209-213.

152. Bajunirwe F, Tisch DJ, King CH, Arts EJ, Debanne SM, Sethi AK. Quality of life and social support among patients receiving antiretroviral therapy in Western Uganda. AIDS Care. Mar 2009;21(3):271279.

153. Huis In 'T Veld D, Pengpid S, Colebunders R, Peltzer K. Body Mass Index and Waist Circumference in Patients with HIV in South Africa and Associated Socio-demographic, Health Related and Psychosocial Factors. AIDS Behav. 2018;22(6):1972-1986.

154. Castro ADCO, Silveira EA, Falco MDO, Nery MW, Turchi MD. Overweight and abdominal obesity in adults living with HIV/AIDS. Revista da Associação Médica Brasileira. 2016;62(4):353-360.

155. Crum-Cianflone N, Tejidor R, Medina S, Barahona I, Ganesan A. Obesity among patients with HIV: the latest epidemic. AIDS Patient Care STDS. Dec 2008;22(12):925-930.

156. Kintu A, Liu E, Hertzmark E, et al. Incidence and Risk Factors for Overweight and Obesity after Initiation of Antiretroviral Therapy in Dar es Salaam, Tanzania. J Int Assoc Provid AIDS Care. Jan-Dec 2018;17:2325958218759759.

157. Thompson-Paul AM, Wei SC, Mattson CL, et al. Obesity Among HIV-Infected Adults Receiving Medical Care in the United States: Data From the Cross-Sectional Medical Monitoring Project and National Health and Nutrition Examination Survey. Medicine (Baltimore). Jul 2015;94(27):e1081.

158. Guehi C, Badjé A, Gabillard D, et al. High prevalence of being Overweight and Obese HIV-infected persons, before and after 24 months on early ART in the ANRS 12136 Temprano Trial. AIDS Res. Ther. 2016;13(1).

159. Okechukwu A, Lawson J, Dalilo M. Dyslipidemia in HIV Infected Children and Adolescents on Highly Active Antiretroviral Therapy in Abuja, Nigeria. J Adv Med Med Res. 2017:1-11.

160. Innes S, Abdullah KL, Haubrich R, Cotton MF, Browne SH. High prevalence of dyslipidemia and insulin resistance in HIV-infected pre-pubertal African children on antiretroviral therapy. Pediatr Infect Dis J. 2016;35(1):e1.

161. Steenkamp L, Truter I, Williams M, et al. Nutritional status and metabolic risk in HIV-infected university students: challenges in their monitoring and management. S Afr Fam Pract (2004). 2017;59(1):9-13. 
162. Mulligan K, Harris DR, Monte D, et al. Obesity and dyslipidemia in behaviorally HIV-infected young women: Adolescent Trials Network study 021. Clin Infect Dis. Jan 1 2010;50(1):106-114.

163. Arbeitman LE, O'Brien RC, Somarriba G, et al. Body mass index and waist circumference of HIVinfected youth in a Miami cohort: comparison to local and national cohorts. J Pediatr Gastroenterol Nutr. 2014 Oct;59(4):449.

164. Bakal DR, Coelho LE, Luz PM, et al. Obesity following ART initiation is common and influenced by both traditional and HIV-/ART-specific risk factors. J Antimicrob Chemother. Aug 1 2018;73(8):21772185.

165. Souza DT, Rondo PHC, Reis LC. The Nutritional Status of Children and Adolescents with HIV/AIDS on Antiretroviral Therapy. J.Trop.Pediatr. 2011;57(1):65-68.

166. Dimala CA, Ngu RC, Kadia BM, Tianyi FL, Choukem SP. Markers of adiposity in HIV/AIDS patients: Agreement between waist circumference, waist-to-hip ratio, waist-to-height ratio and body mass index. PLoS One. 2018;13(3):e0194653.

167. Zimmet P, Alberti KGM, Kaufman F, et al. The metabolic syndrome in children and adolescents ? an IDF consensus report. Pediatr Diabetes. 2007;8(5):299-306.

168. Savva S, Tornaritis M, Savva M, et al. Waist circumference and waist-to-height ratio are better predictors of cardiovascular disease risk factors in children than body mass index. Int $\mathrm{J}$ Obes (Lond). 2000;24(11):1453-1458.

169. Vreeman RC, McCoy BM, Lee S. Mental health challenges among adolescents living with HIV. J Int AIDS Soc. May 16 2017;20(Suppl 3):21497.

170. Binagwaho A, Fawzi MCS, Agbonyitor M, et al. Validating the Children's Depression Inventory in the context of Rwanda. BMC Pediatr. Feb 22 2016;16:29.

171. Lwidiko A, Kibusi SM, Nyundo A, Mpondo BCT. Association between HIV status and depressive symptoms among children and adolescents in the Southern Highlands Zone, Tanzania: A casecontrol study. PLoS One. 2018;13(2):e0193145.

172. Musisi S, Kinyanda E. Emotional and behavioural disorders in HIV seropositive adolescents in urban Uganda. East Afr Med J. 2009;86(1).

173. Petersen AC, Sarigiani PA, Kennedy RE. Adolescent depression: Why more girls? J Youth Adolesc. 1991;20(2):247-271.

174. Nolen-Hoeksema S, Girgus JS. The emergence of gender differences in depression during adolescence. Psychol Bull. 1994;115(3):424.

175. Marhefka SL, Lyon M, Koenig LJ, et al. Emotional and behavioral problems and mental health service utilization of youth living with HIV acquired perinatally or later in life. AIDS Care. Nov 2009;21(11):1447-1454.

176. Hazra R, Siberry GK, Mofenson LM. Growing up with HIV: children, adolescents, and young adults with perinatally acquired HIV infection*. Annu. Rev. Med. 2010;61:169-185. 
177. Buckley J, Otwombe K, Joyce C, et al. Mental Health of Adolescents in the Era of Antiretroviral Therapy: Is There a Difference Between HIV-Infected and Uninfected Youth in South Africa?. J Adolesc Health. Apr 52020.

178. Oladeji BD, Taiwo B, Mosuro O, et al. Suicidal Behavior and Associations with Quality of Life among HIV-Infected Patients in Ibadan, Nigeria. J Int Assoc Provid AIDS Care. Jul/Aug 2017;16(4):376-382.

179. Kinyanda E, Nakasujja N, Levin J, et al. Major depressive disorder and suicidality in early HIV infection and its association with risk factors and negative outcomes as seen in semi-urban and rural Uganda. J Affect Disord. Apr 1 2017;212:117-127.

180. Casale M, Boyes M, Pantelic M, Toska E, Cluver L. Suicidal thoughts and behaviour among South African adolescents living with HIV: Can social support buffer the impact of stigma? J Affect Disord. Feb 15 2019;245:82-90.

181. Petersen I, Hancock JH, Bhana A, Govender K, Mental Health Care MOPFI. Closing the treatment gap for depression co-morbid with HIV in South Africa: Voices of afflicted women. Health. 2013;05(03):557-566.

182. Letlape SV, Mokwena K, Oguntibeju O. Knowledge of students attending a high school in pretoria, South Africa, on diet, nutrition and exercise. West Indian Med J. 2010;59(6):633-640.

183. Bourne LT, Lambert EV, Steyn K. Where does the black population of South Africa stand on the nutrition transition? Public Health Nutr. Feb 2002;5(1a):157-162.

184. Choukem S-P, Tochie JN, Sibetcheu AT, Nansseu JR, Hamilton-Shield JP. Overweight/obesity and associated cardiovascular risk factors in sub-Saharan African children and adolescents: a scoping review. Int J Pediatr Endocrinol. 2020;2020:1-13.

185. World Health Organization. Healthy diet. World Health Organization. Regional Office for the Eastern Mediterranean;2019.

186. Ashdown-Franks G, Vancampfort D, Firth J, et al. Association of leisure-time sedentary behavior with fast food and carbonated soft drink consumption among 133,555 adolescents aged 12-15 years in 44 low- and middle-income countries. Int. J. Behav. Nutr. Phys. Act. 2019;16(1).

187. Malik VS, Popkin BM, Bray GA, Despres JP, Willett WC, Hu FB. Sugar-Sweetened Beverages and Risk of Metabolic Syndrome and Type 2 Diabetes: A meta-analysis. Diabetes Care. 2010;33(11):24772483.

188. National Assembly Republic of South Africa. Foodstuffs, Cosmetics and Disinfectants Act of 1972. Regulations: Reduction of sodium in certain foodstuffs and related matters. Africa GoS, ed. $R$. 533/2012: Government Gazette; 2012.

189. Arthur R. South Africa introduces sugar tax. Beverage Daily, Available at: www. beveragedaily. com/Article/2018/04/03/South-Africa-introduces-sugar-tax.2018 Accessed 30 January 2019.

190. Myers A, Fig D, Tugendhaft A, Mandle J, Myers J, Hofman K. Sugar and health in South Africa: Potential challenges to leveraging policy change. Glob Public Health. 2017;12(1):98-115.

191. Van Biljon A, McKune AJ, Dubose KD, Kolanisi U, Semple SJ. Physical activity levels in urban-based South African learners: A cross-sectional study of 7348 participants. S. Afr. Med. J. 2018;108(2):126. 
192. McVeigh J, Meiring R. Physical activity and sedentary behavior in an ethnically diverse group of South African school children. J Sports Sci Med. 2014;13(2):371.

193. Tanaka LF, Latorre Mdo R, Silva AM, Konstantyner TC, Peres SV, Marques HH. [High prevalence of physical inactivity among adolescents living with HIV/Aids]. Rev Paul Pediatr. Jul-Sep 2015;33(3):327-332.

194. Martins PC, Lima LRA, Teixeira DM, Carvalho AP, Petroski EL. PHYSICAL ACTIVITY AND BODY FAT IN ADOLESCENTS LIVING WITH HIV: A COMPARATIVE STUDY. Rev Paul Pediatr. Jan-Mar 2017;35(1):69-77.

195. Combrink A, Irwin N, Laudin G, Naidoo K, Plagerson S, Mathee A. High prevalence of hookah smoking among secondary school students in a disadvantaged community in Johannesburg. S. Afr. Med. J. 2010;100(5):297-299.

196. Kader Z, Roman NV, Crutzen R. Determinants of Adolescent Hookah Pipe Use: A Systematic Review. J Child Adolesc Subst Abuse. 2020:1-20.

197. Starks TJ, MacDonell KK, Pennar AL, Dinaj-Koci V, Millar BM, Naar S. Drug Use Among Adolescents and Young Adults with Unsuppressed HIV Who Use Alcohol: Identifying Patterns of Comorbid Drug Use and Associations with Mental Health. AIDS Behav. Apr 42020.

198. Alperen J, Brummel S, Tassiopoulos K, Mellins CA, Kacanek D, Smith R, Seage III GR, Moscicki AB. Prevalence of and risk factors for substance use among perinatally human immunodeficiency virusinfected and perinatally exposed but uninfected youth. J Adolesc Health. Mar 2014;54(3):341-349.

199. Sainz T, Diaz L, Navarro ML, et al. Cardiovascular biomarkers in vertically HIV-infected children without metabolic abnormalities. Atherosclerosis. Apr 2014;233(2):410-414.

200. Escota G, Onen N. HIV-infected adolescent, young adult and pregnant smokers: important targets for effective tobacco control programs. Int. J. Environ. Res. Public Health. Jun 2013;10(6):2471-2499.

201. Rossouw TM, Anderson R, Feldman C. Impact of HIV infection and smoking on lung immunity and related disorders. Eur Respir J. Dec 2015;46(6):1781-1795.

202. Triant VA, Lee H, Hadigan C, Grinspoon SK. Increased acute myocardial infarction rates and cardiovascular risk factors among patients with human immunodeficiency virus disease. J. Clin. Endocrinol. Metab. 2007;92(7):2506-2512.

203. Gutierrez J, Elkind MS, Marshall RS. Cardiovascular profile and events of US adults 20-49 years with HIV: results from the NHANES 1999-2008. AIDS Care. 2013;25(11):1385-1391.

204. Falk DE, Yi H-y, Hiller-Sturmhöfel S. An epidemiologic analysis of co-occurring alcohol and tobacco use and disorders: findings from the National Epidemiologic Survey on Alcohol and Related Conditions. Alcohol Research \& Health. 2006;29(3):162.

205. Mellins CA, Tassiopoulos K, Malee K, et al. Behavioral health risks in perinatally HIV-exposed youth: co-occurrence of sexual and drug use behavior, mental health problems, and nonadherence to antiretroviral treatment. AIDS Patient Care STDS. Jul 2011;25(7):413-422.

206. Toska E, Pantelic M, Meinck F, Keck K, Haghighat R, Cluver L. Sex in the shadow of HIV: A systematic review of prevalence, risk factors, and interventions to reduce sexual risk-taking among HIV-positive 
adolescents and youth in sub-Saharan Africa. PLOS ONE. 2017;12(6):e0178106.

207. Harker N, Kader R, Myers B, et al. Substance abuse trends in the Western Cape: A review of studies conducted since 2000. 2008.

208. Weybright EH, Caldwell LL, Wegner L, Smith E, Jacobs J. The state of methamphetamine ('tik') use among youth in the Western Cape, South Africa. S. Afr. Med. J. 2016;106(11):1125-1128.

209. Kagura J, Adair LS, Musa MG, Pettifor JM, Norris SA. Blood pressure tracking in urban black South African children: birth to twenty cohort. BMC pediatrics. 2015;15(1):78.

210. Naidoo S, Kagura J, Fabian J, Norris SA. Early Life Factors and Longitudinal Blood Pressure Trajectories Are Associated With Elevated Blood Pressure in Early Adulthood. Hypertension. 2019;73(2):301-309.

211. Chatterton-Kirchmeier S, Camacho-Gonzalez AF, McCracken CE, Chakraborty R, Batisky DL. Increased prevalence of elevated blood pressures in HIV-infected children, adolescents and young adults. The Pediatric infectious disease journal. Jun 2015;34(6):610-614.

212. Fahme SA, Bloomfield GS, Peck R. Hypertension in HIV-Infected Adults. Hypertension. 2018;72(1):4455.

213. Nduka C, Stranges S, Sarki A, Kimani P, Uthman O. Evidence of increased blood pressure and hypertension risk among people living with HIV on antiretroviral therapy: a systematic review with meta-analysis. J Hum Hypertens. 2016;30(6):355-362.

214. Pangmekeh PJ, Awolu MM, Gustave S, Gladys T, Cumber SN. Association between highly active antiretroviral therapy (HAART) and hypertension in persons living with HIV/AIDS at the Bamenda regional hospital, Cameroon. Pan Afr Med J. 2019;33:87.

215. Järvelin M-R, Sovio U, King V, et al. Early life factors and blood pressure at age 31 years in the 1966 northern Finland birth cohort. Hypertension. 2004;44(6):838-846.

216. Prioreschi A, Munthali RJ, Soepnel L, et al. Incidence and prevalence of type 2 diabetes mellitus with HIV infection in Africa: a systematic review and meta-analysis. BMJ open. Mar 29 2017;7(3):e013953.

217. Brown TT, Cole SR, Li X, et al. Antiretroviral therapy and the prevalence and incidence of diabetes mellitus in the multicenter AIDS cohort study. Archives of internal medicine. 2005;165(10):11791184.

218. De Wit S, Sabin CA, Weber R, et al. Incidence and risk factors for new-onset diabetes in HIV-infected patients the data collection on adverse events of anti-HIV drugs (D: A: D) study. Diabetes Care. 2008;31(6):1224-1229.

219. Tripathi A, Liese AD, Jerrell JM, et al. Incidence of diabetes mellitus in a population-based cohort of HIV-infected and non-HIV-infected persons: the impact of clinical and therapeutic factors over time. Diabet Med. Oct 2014;31(10):1185-1193.

220. International Diabetes Federation. IDF diabetes atlas ninth edition 2019. 2019. 
221. Jemmott JB, 3rd, Jemmott LS, O'Leary A, et al. Cognitive-behavioural health-promotion intervention increases fruit and vegetable consumption and physical activity among South African adolescents: a cluster-randomised controlled trial. Psychol Health. Feb 2011;26(2):167-185.

222. Woollett N, Peter J, Cluver L, Brahmbhatt $\mathrm{H}$. Enrolling HIV-positive adolescents in mental health research: A case study reflecting on legal and ethical complexities. S Afr Med J. Jul 28 2017;107(8):679-683.

223. Oliveras C, Cluver L, Bernays S, Armstrong A. Nothing About Us Without RIGHTS-Meaningful Engagement of Children and Youth: From Research Prioritization to Clinical Trials, Implementation Science, and Policy. J Acquir Immune Defic Syndr. Aug 15 2018;78 Suppl 1(1):S27-s31.

224. Lee PH, Macfarlane DJ, Lam T, Stewart SM. Validity of the international physical activity questionnaire short form (IPAQ-SF): A systematic review. Int. J. Behav. Nutr. Phys. Act. 2011;8(1):115.

225. Ekelund U, Sepp H, Brage S, et al. Criterion-related validity of the last 7-day, short form of the International Physical Activity Questionnaire in Swedish adults. Public Health Nutr. 2006;9(2):258265.

226. Steene-Johannessen J, Anderssen SA, Van der Ploeg HP, et al. Are self-report measures able to define individuals as physically active or inactive? Med Sci Sports Exerc. 2016;48(2):235.

227. Corder K, Van Sluijs EMF. Invited Commentary: Comparing Physical Activity Across Countries-Current Strengths and Weaknesses. Am. J. Epidemiol. 2010;171(10):1065-1068.

228. Engelgau MM, Narayan KM, Herman WH. Screening for type 2 diabetes. Diabetes Care. 2000;23(10):1563-1580.

229. Engelgau MM, Thompson TJ, Smith PJ, et al. Screening for diabetes mellitus in adults: the utility of random capillary blood glucose measurements. Diabetes Care. 1995;18(4):463-466.

230. Baron EC, Davies T, Lund C. Validation of the 10-item centre for epidemiological studies depression scale (CES-D-10) in Zulu, Xhosa and Afrikaans populations in South Africa. BMC Psychiatry. 2017;17(1):6.

231. Dupont A, Wheeler J, Herndon J, et al. Use of tablet personal computers for sensitive patient-reported information. J Support Oncol. 2009;7(3):91-97.

232. Jaspan HB, Flisher AJ, Myer L, et al. Brief report: Methods for collecting sexual behaviour information from South African adolescents-a comparison of paper versus personal digital assistant questionnaires. J Adolesc. 2007;30(2):353-359.

\section{Figures}




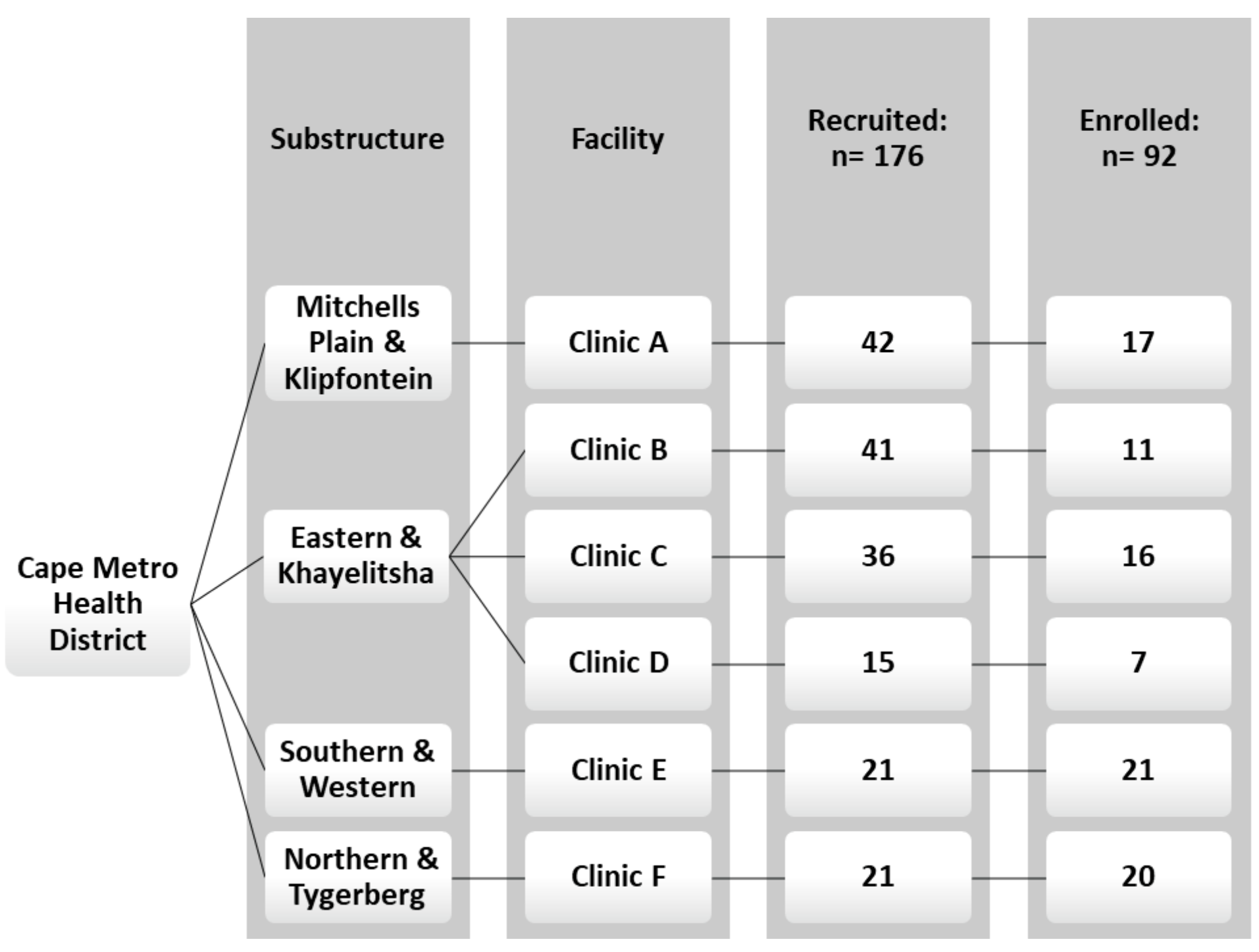

Figure 1

Participant Recruitment and enrolment at respective facilities 


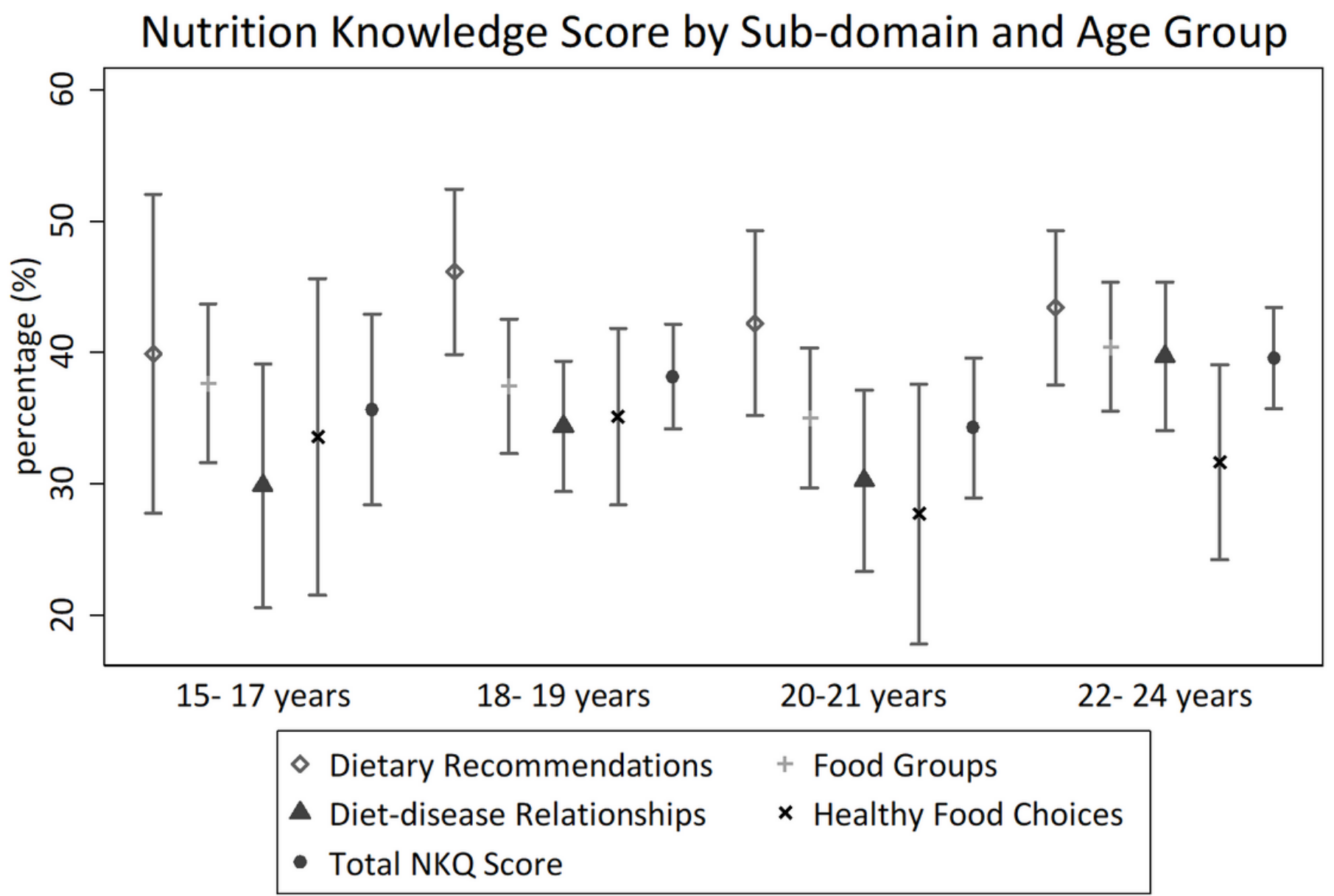

$95 \%$ confidence intervals

Figure 2

Nutrition Knowledge Score by Sub-domain and Age Group 
Risk Behaviours by Gender and Age

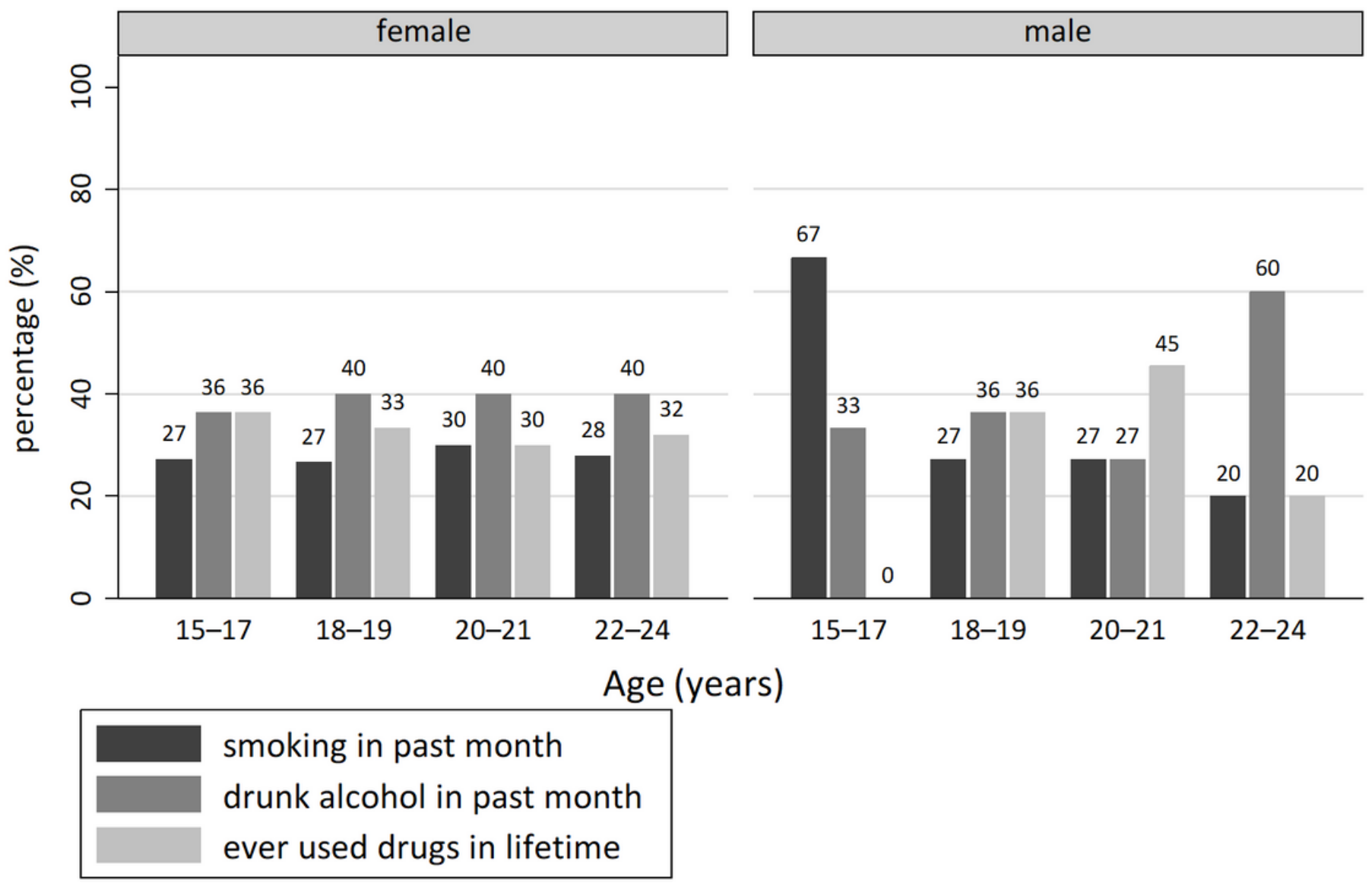

Figure 3

Current smoking, alcohol consumption, and lifetime substance use by Gender and Age Group 
CESD-10 Depression by Gender and Age

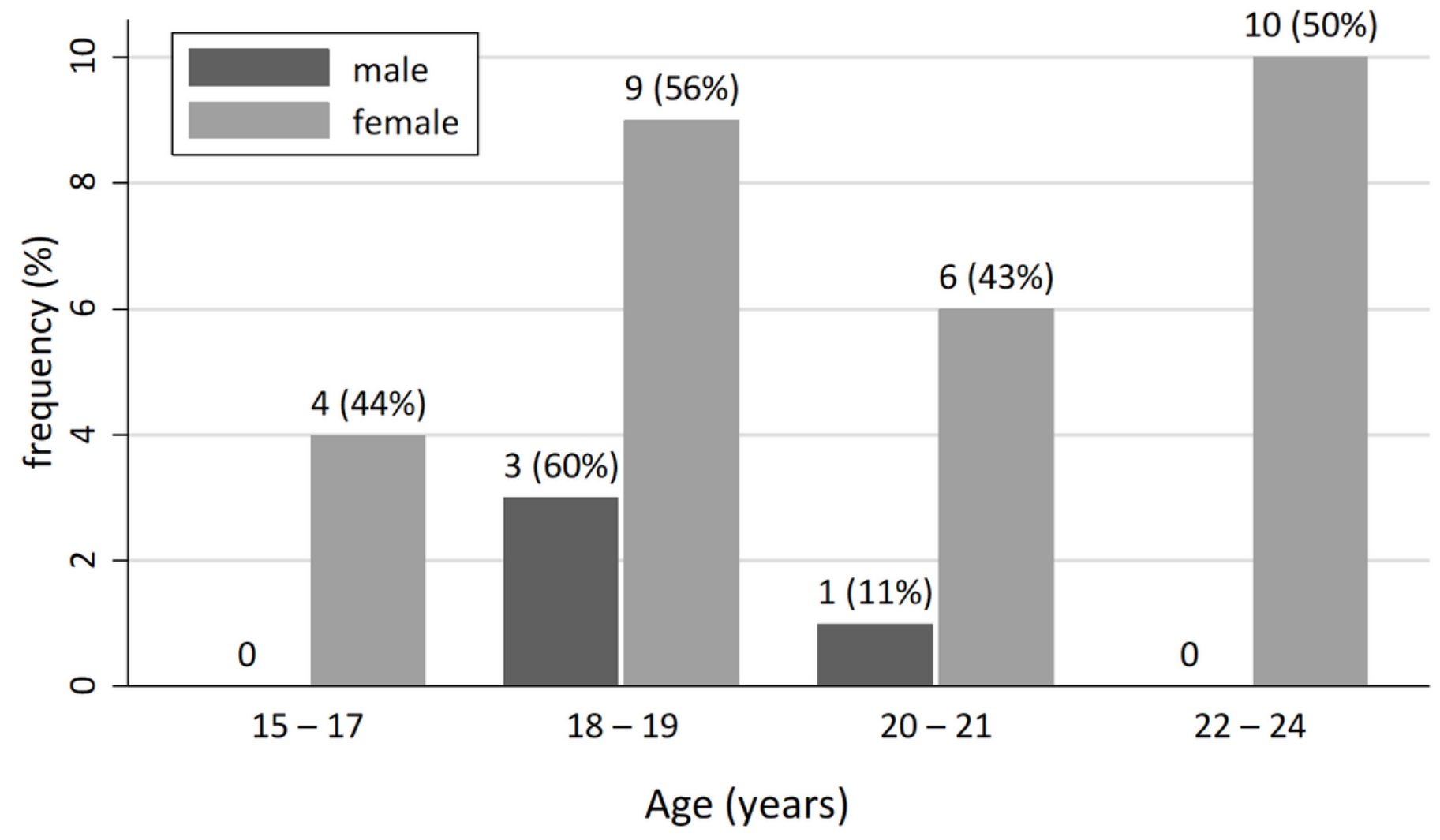

Figure 4

Mental Health Status by Gender and Age Group Bar represents proportion (\%) with significant depression (CESD score $\geq 10$ ) by gender in each age group. 


\section{Psychological Distress by Gender and Age}

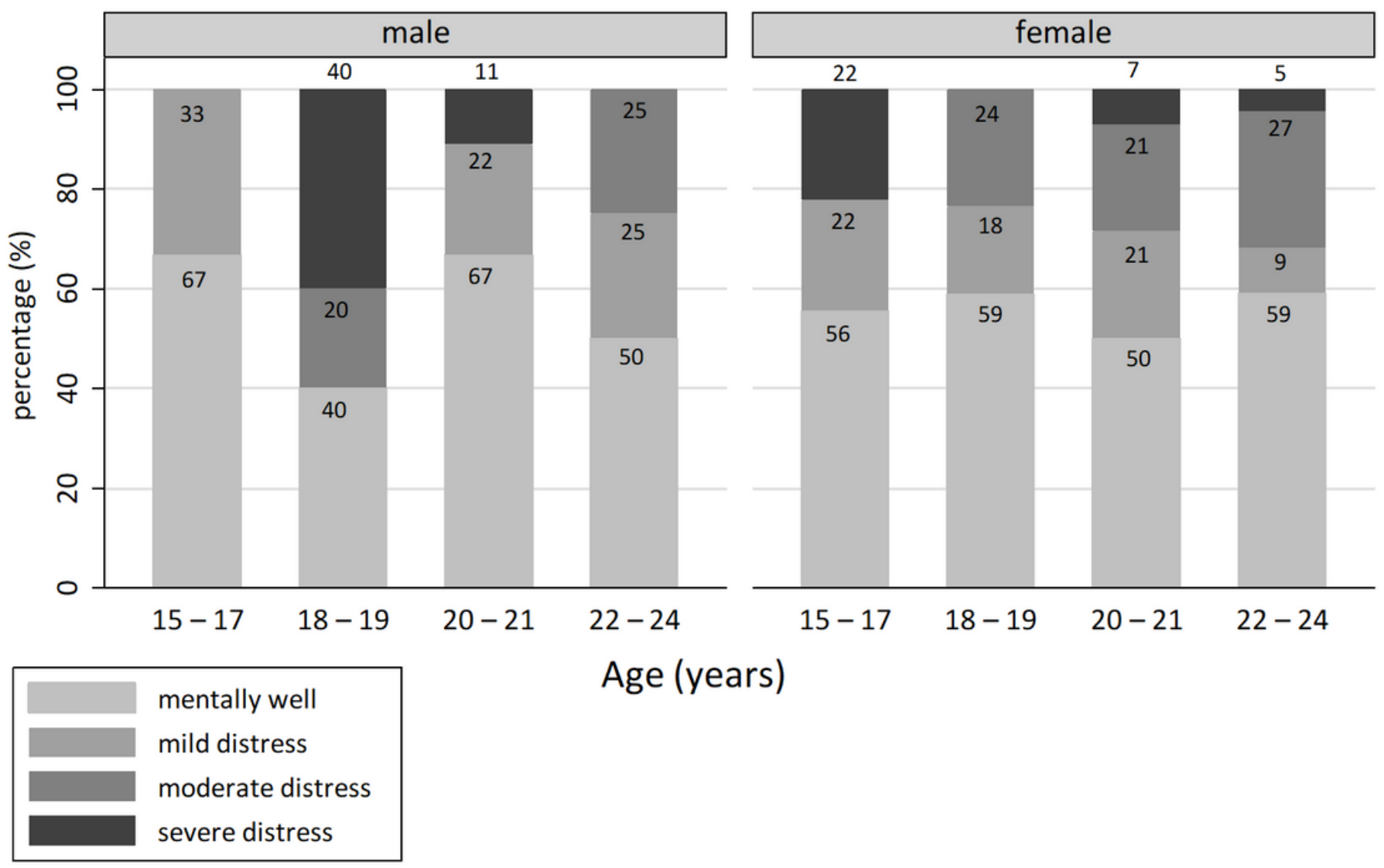

Figure 5

Severity of Psychological Distress by Gender and Age Group Psychological distress according to Kessler (K10) Distress Scale. Bars represent severity of distress (\%) by gender and age group 


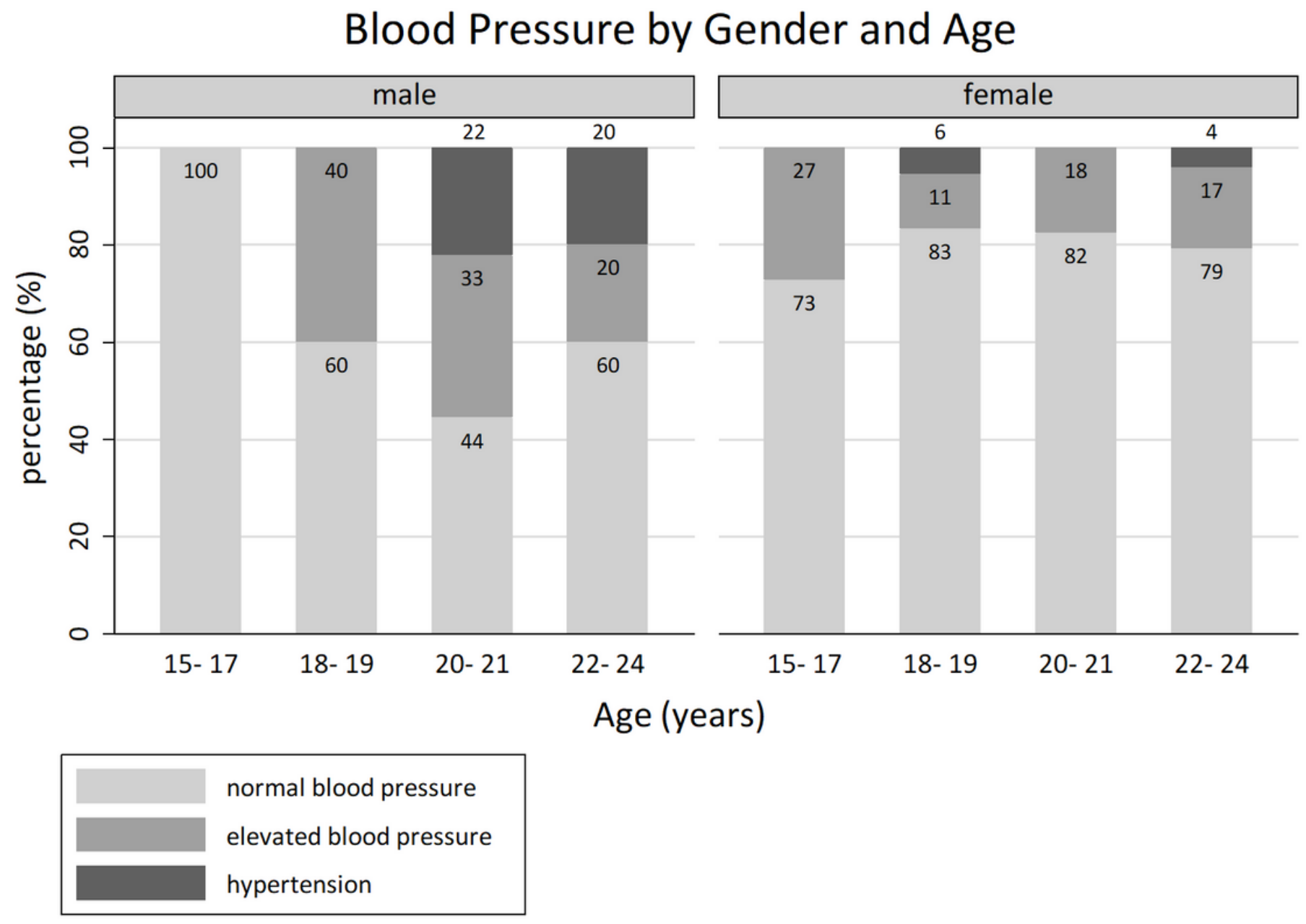

Figure 6

Blood Pressure Category by Gender and Age Group 


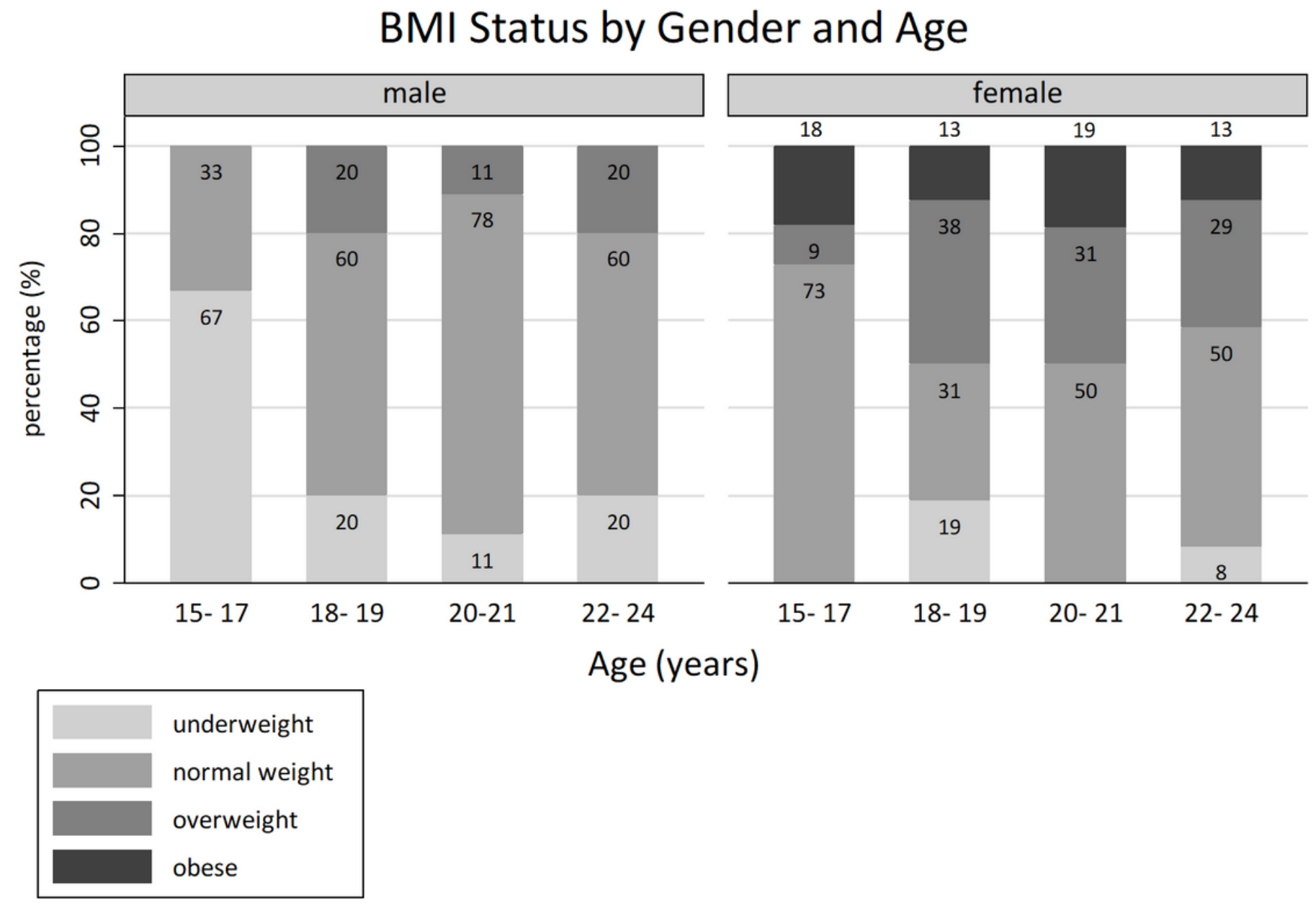

Figure 7

Weight Status by Gender and Age Group 